\title{
A census of massive stars in NGC $346^{\star}$ Stellar parameters and rotational velocities
}

\author{
P. L. Dufton ${ }^{1}$, C. J. Evans ${ }^{2}$, I. Hunter ${ }^{1}$, D. J. Lennon ${ }^{3}$, and F. R. N. Schneider ${ }^{4,5}$
}

\author{
1 Astrophysics Research Centre, School of Mathematics \& Physics, The Queen's University of Belfast, BT7 1NN Belfast, UK \\ e-mail: p.dufton@qub.ac.uk \\ 2 UK Astronomy Technology Centre, Royal Observatory, Blackford Hill, Edinburgh EH9 3HJ, UK \\ ${ }^{3}$ Instituto de Astrofísica de Canarias, 38205 La Laguna, Tenerife, Spain \\ ${ }^{4}$ Zentrum für Astronomie der Universität Heidelberg, Astronomisches Rechen-Institut, Mönchhofstr. 12-14, \\ 69120 Heidelberg, Germany \\ ${ }^{5}$ Heidelberger Institut für Theoretische Studien, Schloss-Wolfsbrunnenweg 35, 69118 Heidelberg, Germany
}

Received 6 March 2019 / Accepted 7 May 2019

\begin{abstract}
Spectroscopy for 247 stars towards the young cluster NGC 346 in the Small Magellanic Cloud has been combined with that for 116 targets from the VLT-FLAMES Survey of Massive Stars. Spectral classification yields a sample of 47 O-type and 287 B-type spectra, while radial-velocity variations and/or spectral multiplicity have been used to identify 45 candidate single-lined (SB1) systems, 17 double-lined (SB2) systems, and one triple-lined (SB3) system. Atmospheric parameters $\left(T_{\text {eff }}\right.$ and $\left.\log g\right)$ and projected rotational velocities $\left(v_{\mathrm{e}} \sin i\right)$ have been estimated using TLUSTY model atmospheres; independent estimates of $v_{\mathrm{e}} \sin i$ were also obtained using a Fourier Transform method. Luminosities have been inferred from stellar apparent magnitudes and used in conjunction with the $T_{\text {eff }}$ and $v_{\mathrm{e}} \sin i$ estimates to constrain stellar masses and ages using the BonNsaI package. We find that targets towards the inner region of NGC 346 have higher median masses and projected rotational velocities, together with smaller median ages than the rest of the sample. There appears to be a population of very young targets with ages of less than $2 \mathrm{Myr}$, which have presumably all formed within the cluster. The more massive targets are found to have lower projected rotational velocities consistent with previous studies. No significant evidence is found for differences with metallicity in the stellar rotational velocities of early-type stars, although the targets in the Small Magellanic Cloud may rotate faster than those in young Galactic clusters. The rotational velocity distribution for single non-supergiant B-type stars is inferred and implies that a significant number have low rotational velocity ( $\simeq 10 \%$ with $\left.v_{\mathrm{e}}<40 \mathrm{~km} \mathrm{~s}^{-1}\right)$, together with a peak in the probability distribution at $v_{\mathrm{e}} \simeq 300 \mathrm{~km} \mathrm{~s}^{-1}$. Larger projected rotational velocity estimates have been found for our Be-type sample and imply that most have rotational velocities between $200-450 \mathrm{~km} \mathrm{~s}^{-1}$.
\end{abstract}

Key words. stars: early-type - stars: atmospheres - stars: rotation - stars: evolution - Magellanic Clouds open clusters and associations: individual: NGC 346

\section{Introduction}

Massive stars significantly influence the evolution of their host clusters and galaxies via feedback of both energy and chemically-processed material. To help reconcile evolutionary predictions of massive stars with observations, the effects of stellar rotation have been included in theoretical models (Heger \& Langer 2000; Meynet \& Maeder 2000) and applied to, for example, understanding the ratios of red-to-blue supergiants (Maeder \& Meynet 2001) and the populations of Wolf-Rayet stars (Meynet \& Maeder 2005; Vink \& de Koter 2005).

One of the primary motivations for including rotation in massive-star models were observations of core-processed material, for example enhanced nitrogen abundances, on the stellar surface (see, e.g. Walborn 1970; Gies \& Lambert 1992; Venn 1999; Bouret et al. 2003; Lennon et al. 2003; Korn et al. 2002, 2005). The process of rotational mixing then naturally explained how material could be mixed from the core to the surface. Additionally, rapid rotation may be a prerequisite in producing a

\footnotetext{
* Based on observations at the European Southern Observatory in programmes 171.D-0237 and 074D.0011.
}

gamma-ray burst from a single massive star via its homogenous evolution (Yoon \& Langer 2005; Woosley \& Heger 2006).

A large observational sample of $\sim 500$ OB-type stars in the Galaxy and Magellanic Clouds was obtained by the FLAMES Survey of Massive Stars (FSMS; Evans et al. 2005, 2006) to investigate these topics. Surface nitrogen abundances estimated for the B-type samples in the FSMS (Hunter et al. 2007, 2008a,b; Trundle et al. 2007) implied rotational mixing might not be the only transport mechanism involved, and the nature of these stars has been discussed further by, for example, Brott et al. (2011a), Maeder et al. (2014) and Aerts et al. (2014). O-type stars with low projected rotational velocities and abundances that appear inconsistent with rotational mixing were also identified by Rivero González et al. (2012).

In a second campaign, the VLT-FLAMES Tarantula Survey (VFTS; Evans et al. 2011), spectroscopy was obtained for $\sim 800$ targets in the 30 Doradus region of the Large Magellanic Cloud (LMC). Targets with enhanced surface nitrogen abundances, which appeared to be incompatible with current singlestar models of rotational mixing, were again identified in both the O-type (Grin et al. 2017) and B-type (Dufton et al. 2018) populations. Definitive conclusions have been hampered both by 
observational and theoretical uncertainties, and by the possibility of other evolutionary scenarios, such as binarity and magnetic fields (see Grin et al. 2017; Dufton et al. 2018, for further details). Nevertheless, rotation remains one of the critical parameters in the evolution of massive stars.

Theoretical models predict that massive stars at higher metallicity have stronger line-driven winds, thereby losing more mass and angular momentum over their lifetime compared to those at lower metallicities (Kudritzki et al. 1987; Kudritzki \& Puls 2000; Vink \& de Koter 2005; Mokiem et al. 2006). However, studies of rotational velocities of early-type star from ultraviolet spectroscopy have found little compelling evidence for stars in the Clouds rotating more quickly than in the Galaxy (Penny et al. 2004; Penny \& Gies 2009). Hunter et al. (2008a) presented rotational velocities for $\sim 400$ massive stars from optical spectroscopy in both the LMC and the Small Magellanic Cloud (SMC) and compared these with Galactic field stars to show that the predicted trend of rotational velocity and metallicity was observable. However, the analysis was complicated by the nature of the samples. Although the target stars in the Clouds were in the direction of young clusters, much of the sample was composed of field stars. Galactic studies have implied that stars in clusters rotate faster than those in the field (see, e.g. Strom et al. 2005; Dufton et al. 2006a; Huang \& Gies 2006; Wolff et al. 2007), with a similar trend observed in the LMC (Keller 2004; Wolff et al. 2008). This would be consistent with the stars in the older field populations having spun down over their lifetimes. Alternatively, as suggested by Wolff et al. (2007), the star formation process may be affected by the properties of the ambient gas, with stars in clusters being born from more energetic material and having shorter lived magnetically-locked accretion discs.

Analysis of the VFTS data has yielded estimates of the rotational velocity distributions and atmospheric parameters for both the O-type (Ramírez-Agudelo et al. 2013, 2015; Grin et al. 2017) and B-type (Dufton et al. 2013, 2018; Garland et al. 2017) samples in the 30 Doradus region in the LMC. Here we present complementary observations of massive stars in the region of the cluster NGC 346 in the lower-metallicity environment of the SMC. Our observations can be combined with those for earlytype stars previously observed towards this cluster by the FSMS. By using the same observational settings and tools as in the analysis of the FSMS and VFTS data we can minimise any systematic effects in the comparison of their fundamental parameters.

The observational material is presented in Sect. 2 and its spectral classification discussed in Sect. 3 and Appendix A. Section 4 describes the methods used to estimate the atmospheric parameters, projected rotational velocities, luminosity, masses and ages for our targets, which are then discussed in Sect. 5.

\section{Observations}

Spectroscopy was obtained using the Fibre Large Array MultiElement Spectrgraph (FLAMES; Pasquini et al. 2002) on the Very Large Telescope at the European Southern Observatory (ESO). The FLAMES-Medusa mode was used to feed light from typically 80 stellar targets (plus sky fibres) per fibre configuration to the Giraffe spectrograph. Our primary aim was to obtain a near-complete spectroscopic census of the O-type and early B-type populations of the NGC 346 region, with targets selected from the ESO Imaging Survey (EIS; Momany et al. 2001). Hereafter this spectroscopy will be designated the Survey dataset to distinguish it from the FSMS data.

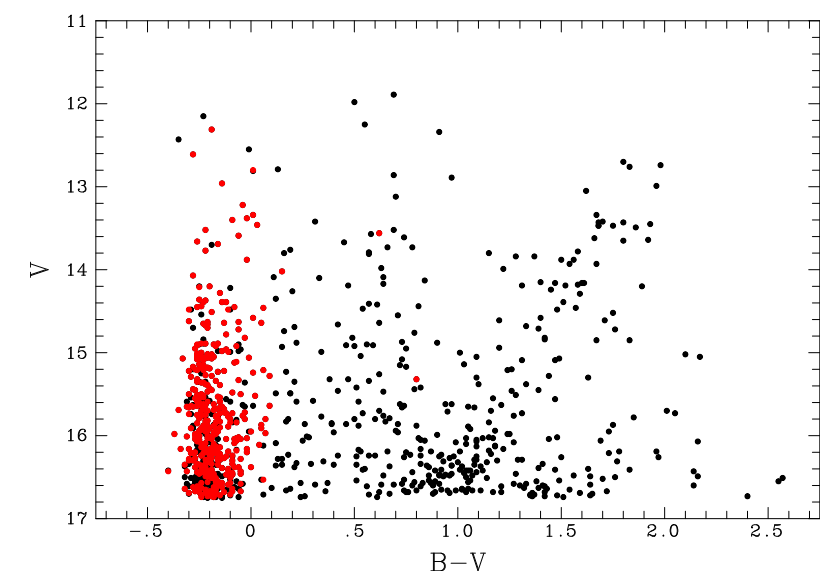

Fig. 1. Colour-magitude diagram showing the location of the additional FLAMES targets combined with those from the FSMS (363 stars, red points) compared to all potential targets $(V \leq 16.75)$ within a $10^{\prime}$ search radius of the centre of the cluster.

A faint magnitude cut-off of $V \leq 16.75$ mag was employed to ensure that the signal-to-noise ratios of the spectra were sufficient for quantitative analysis. Adopting a distance modulus to the SMC of 18.9 (Harries et al. 2003; Hilditch et al. 2005), this corresponds to a latest (unreddened, main sequence) spectral type of approximately B3 V (cf. Walborn 1972). A colour cut of $(B-V) \leq 0.1$ was also employed to restrict the sample to OB-type stars, after allowing for a typical interstellar reddening for NGC 346 of $E(B-V) \sim 0.08$ (e.g. Hennekemper et al. 2008); similar criteria were previously employed in the selection of the FSMS targets. A colour-magnitude diagram for our targets and for the potential targets from the EIS catalogue (within a 10' search radius of the cluster centre) is shown in Fig. $1^{1}$.

Astrometry and optical photometry for our targets are summarised in Table A.1. Identifications (sorted by $V$-band magnitude) have been assigned from \#1001 to distinguish them from the FSMS targets; similar information for the latter is given in Table 4 of Evans et al. (2006). Also included in Table A.1 are radial distances of each target from \#1001 (the brightest object in the central part of the cluster), following the approach in Table 1 of Hunter et al. (2008a).

The spatial distribution of our targets and those previously observed by the FSMS in the central region of NGC 346 are shown in Fig. 2. The FLAMES-Medusa fibres project to a diameter of 1 !'2 on the sky, equivalent to $0.35 \mathrm{pc}$ in the SMC, and therefore may include contributions from companions unresolved in the ground-based imaging. From inspection of Hubble Space Telescope (HST) images of NGC 346 (Sabbi et al. 2007), in all but one instance (\#0111) our targets are by far the brightest source in the fibre aperture. This comparison was only possible for 82 of our 363 sources due to the limited extent of the HST imaging. However it includes the densest regions at the centre of the field (Fig. 2) where these effects might be expected to be most significant.

The radius of the ionised region of NGC 346 has been given as 3.5 by Relaño et al. (2002) which, at the distance of the SMC, corresponds to a physical distance of $61 \mathrm{pc}$. Given the magnitude cut-off and colour selection, 125 stars from 167 potential targets were observed out to this radius, a completeness of $75 \%$

1 Two "red" stars were also observed. One is NGC 346-003 from the FSMS, observed with the fibre-feed to UVES (see Evans et al. 2006). The other (\#1103) had spurious EIS photometry and was later supplemented by archival photometry (see Table A.1). 


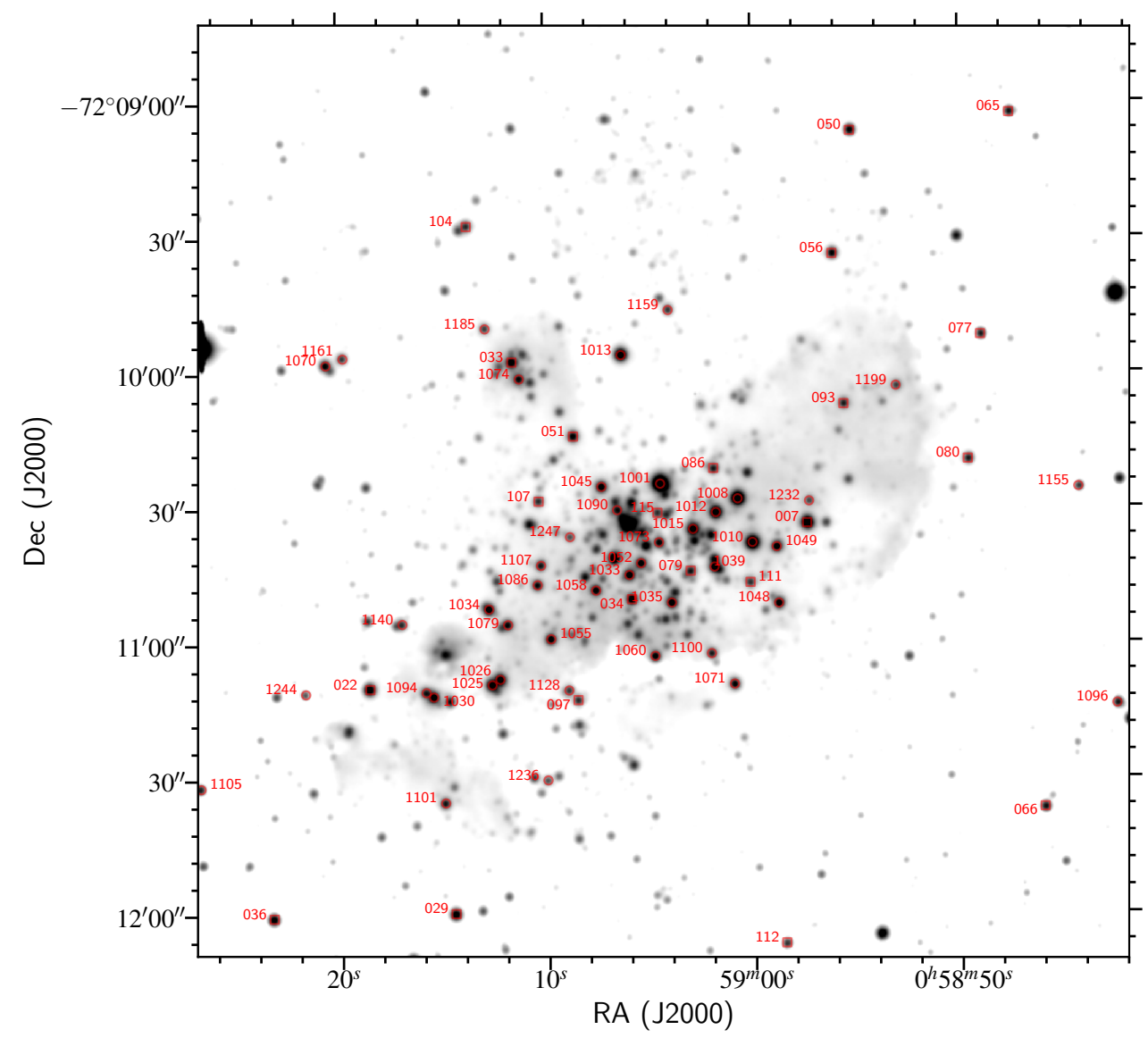

Fig. 2. FLAMES targets in the central $\sim 3$ '. 5 of NGC 346 . Targets with identifications $>1000$ are from the observations presented here, the remainder are from Evans et al. (2006). which rises to $88 \%$ for $V<16.0$. The principal limitation on the completeness was the crowding of targets in the core of the cluster, and the minimum approach distance permitted for the Medusa fibre-heads. Nearly all of the bright stars in the central region without FLAMES spectroscopy have previous spectroscopy, leading for the first time to a comprehensive census of the high-mass spectroscopic content of NGC 346.

The observations were taken in service mode between 2004 September 27 and November 28. Three Medusa configurations with near-identical central positions (Fields "A","B" and "C") were observed as summarised in Table 1. Two of the standard Giraffe settings were used: LR02 (with a wavelength range of $3960-4564 \AA$ at a spectral resolving power of $R \sim 7000)$ and LR03 (4499-5071 $\AA, R \sim 8500$ ). These gave full coverage of the blue optical region, with an overlap from $\lambda \lambda 4500-4565 \AA$. At least four exposures at each grating setting were taken for each field. In some cases the requirements on the observing conditions were not satisfied and observing blocks were repeated. As a consequence, six exposures were obtained at both settings for Field B, and eight for Field C. Although the time-sampling is not as extensive as that obtained in the FSMS, the observations offer some leverage on the detection of massive binaries.

As for the FSMS data ${ }^{2}$, the Giraffe Base-line Reduction Software (girBLDRS; Blecha, et al. 2003) was used for bias subtraction, flat-field correction, fibre extraction and wavelength calibration. The sky subtraction and correction to the heliocentric velocity frame was then undertaken using the STARLINK software DIPso (Currie et al. 2014).

\footnotetext{
2 For the FSMS targets the normalised spectra were taken directly from the data archive available at: https://star.pst.qub.ac.uk/ sjs/ flames/
}

The multiple spectra of each target were first compared to search for evidence of radial-velocity variations - simple division of the spectra from one epoch into another can reveal "P Cygni"-like features for binary systems. The overlap region between the LR02 and LR03 settings helped in this regard as it contains useful stellar lines (e.g. He II 24542 , Si III 24552) to increase the time cadence. Stars displaying radial-velocity variations significant enough to be detected from this simple approach $\left(\gtrsim 10 \mathrm{~km} \mathrm{~s}^{-1}\right)$ are considered as candidate single-lined binaries (SB1) in the final column of Table A.1. Similarly, targets clearly displaying multiple components are classified as double-lined binaries (SB2), and in one instance as a triple-lined binary (SB3).

For the apparently single stars, all usable spectra were combined either by simple addition or by using a median $\sigma$-clipping algorithm. The final spectra were normally indistinguishable apart from regions affected by cosmic rays where the latter method was superior. The full wavelength range for spectrum at each grating setting could usually be normalised using a single, low-order polynomial. However, for some features (e.g. the Balmer series), the combined spectra around individual (or groups of) lines were separately normalised.

Garland et al. (2017) have discussed the reduction of LR02 and LR03 FLAMES spectroscopy for targets which show significant radial-velocity variations. They found that even for narrow-lined stars (with $v_{\mathrm{e}} \sin i \leq 40 \mathrm{~km} \mathrm{~s}^{-1}$ ), simply combining exposures without velocity shifts led to no significant spectral degradation provided the range of radial velocities, $\Delta v_{\mathrm{r}}$, was less than $30 \mathrm{~km} \mathrm{~s}^{-1}$. For targets with larger projected rotational velocities, the maximum value of $\Delta v_{\mathrm{r}}$ increased, e.g. for $v_{\mathrm{e}} \sin i \sim 120 \mathrm{~km} \mathrm{~s}^{-1}$, it corresponded to $\Delta v_{\mathrm{r}} \leq 100 \mathrm{~km} \mathrm{~s}^{-1}$. Hence for the SB1 candidates, we have initially combined the exposures without any velocity shifts and estimated the 
Table 1. Observing log of the FLAMES NGC 346 Survey observations

\begin{tabular}{lcccc}
\hline \hline Date & Field & Setting & $\begin{array}{c}\lambda_{\mathrm{c}} \\
(\AA)\end{array}$ & $\begin{array}{c}\text { Exposure time } \\
(\mathrm{s})\end{array}$ \\
\hline $2004-09-27$ & A & LR02 & 4272 & $2 \times 1725$ \\
$2004-09-28$ & A & LR02 & 4272 & $2 \times 1725$ \\
$2004-10-01$ & A & LR03 & 4797 & $2 \times 1725$ \\
$2004-10-04$ & A & LR03 & 4797 & $2 \times 1725$ \\
\hline $2004-10-14$ & B & LR02 & 4272 & $1 \times 1725,1 \times 1234$ \\
$2004-10-17$ & B & LR02 & 4272 & $2 \times 1725$ \\
$2004-10-18$ & B & LR03 & 4797 & $4 \times 1725$ \\
$2004-11-25$ & B & LR02 & 4272 & $2 \times 1725$ \\
$2004-11-25$ & B & LR03 & 4797 & $2 \times 1725$ \\
\hline $2004-10-18$ & C & LR02 & 4272 & $4 \times 1725$ \\
$2004-10-18$ & C & LR03 & 4797 & $4 \times 1725$ \\
$2004-11-26$ & C & LR02 & 4272 & $4 \times 1725$ \\
$2004-11-26$ & C & LR03 & 4797 & $2 \times 1725$ \\
$2004-11-28$ & C & LR03 & 4797 & $2 \times 1725$ \\
\hline
\end{tabular}

$v_{\mathrm{e}} \sin i$ as discussed in Sect. 4.2. We have then cross-correlated exposures from individual epochs (see Table 1) to estimate $\Delta v_{\mathrm{r}}$ for each wavelength setting. The $v_{\mathrm{e}} \sin i$ and $\Delta v_{\mathrm{r}}$ estimates for each wavelength setting in each target were then compared to the simulations of Garland et al. (2017). They were generally consistent with no significant spectral degradation, the only exceptions being: \#1014 (LR02 and LR03 settings), \#1023 (LR03), \#1101 (LR02), \#1182 (LR02), \#1192 (LR03), \#1196 (LR02), \#1209 (LR03), \#1241 (LR03), \#1246 (LR03). The spectroscopy for these cases has been re-reduced with the individual exposures shifted in velocity space using the results from the cross-correlations. The projected rotational velocities were reestimated, with the changes in the estimates always being less than 5\%. For the ten additional Survey targets identified as SB2, the exposures for each epoch were combined separately.

\section{Spectral classification}

All the Survey spectroscopy has been classified with reference to criteria developed for massive stars in the low metallicity environment of the SMC (Lennon 1997; Walborn et al. 2000; Evans \& Howarth 2003; Evans et al. 2004). Spectral classifications for all 247 targets are given in Table A. $1^{3}$, together with previous published classifications. Cross-matches with past identifications were generally performed by visual comparison of published finding charts and the EIS images. In the case of the $2 \mathrm{dF}$ spectroscopy from Evans et al. (2004), stars were matched using their astrometric data and a conservative search radius of $1^{\prime \prime}$ (to avoid specious matches). Previous classifications were principally from Massey et al. (1989, 19 stars), and Evans et al. (2004, 18 stars) and are included in the final column of Table A.1 in italics. Other classifications are included as footnotes to the table. Detailed comments on five targets of note are provided in Appendix B.

In general there was good agreement with previous classifications, although a few stars (e.g. \#1048 and \#1058) received later types than before, probably owing to the improved spectral resolution of the FLAMES data. While good nebular subtraction is difficult to achieve in fibre spectroscopy, He I nebular emis-

\footnotetext{
3 These were previously included in the compendium of SMC data by Bonanos et al. (2010) as "Hunter et al., in prep."
}

Table 2. Spectral content of the NGC 346 region from the FSMS (Evans et al. 2006) and the Survey data.

\begin{tabular}{lcccccc}
\hline \hline Source & Total & $\mathrm{O}$ & $\begin{array}{c}\text { Early-B } \\
{[\mathrm{B} 0-3]}\end{array}$ & $\mathrm{Be}$ & $\begin{array}{c}\text { Late-B } \\
{[\mathrm{B} 5-9]}\end{array}$ & AFG \\
\hline FSMS & 116 & 19 & 59 & 25 & 2 & 11 \\
Survey & 247 & 28 & 149 & 45 & 8 & 17 \\
Total & 363 & 47 & 208 & 70 & 10 & 28 \\
\hline
\end{tabular}

Notes. All of the Be-type spectra are of early B-type, except for two classified as B5 in the Survey dataset. \#1024 has been classified as a Betype star but see Appendix A for a detailed discussion of its spectrum.

sion was typically resolved in the cores, highlighting the potential for infilling if degraded to lower resolution. We were also able to refine some of the previously uncertain classifications from Evans et al. (2004).

The Survey data thus provide the first classifications for over 200 massive stars in this important region in the SMC, including ten new O-type stars (six of which are within 1!2 of the centre). When combined with the FSMS spectroscopy from Evans et al. (2006), this represents a detailed spectral census of the NGC 346 cluster and its environs. A summary of the spectral content of the two observational samples is given in Table 2 .

Twelve targets have been classified as O-type binaries (9 SB1, 2 SB2, 1 SB3) and fifty one as B-type binaries (36 SB1, 15 SB2). This translates into observed binary percentages (plus standard deviations assuming binomial statistics) of $26 \pm 6 \%$ (O-type) and $18 \pm 2 \%$ (B-type). For the VFTS survey of the Tarantula Nebula in the LMC, observed binary percentages of $35 \pm 3 \%$ (O-type Sana et al. 2013) and $25 \pm 2 \%$ (B-type Dunstall et al. 2015) were found. However, as discussed by those authors, the actual binary percentages will be larger due to some binaries not having been identified; from Monte-Carlo simulations they inferred actual binary percentages of more than $50 \%$ for both O- and B-type systems. Given the limited time cadence of our spectroscopy, our observed binary fractions appear consistent with those found from the VFTS.

Known omissions from our spectroscopy (due to crowding of the Medusa fibres) in the central part of the cluster include: MPG 395, 451, 468, and 487, with classifications from Massey et al. (1989) of B0 V, B0 V, O9 V, and O6.5 V, respectively; MPG 470, classified as O8-9 III:nw (Walborn \& Blades 1986); the components of N66A, which include N66A-1 (O8 V, Heydari-Malayeri \& Selier 2010); and MPG 375 ( $V=15.47)$, for which the spectral type is unknown. With our focus on OBtype stars, we have also omitted HD 5980, the bright source on the eastern edge of Fig. 2, approx. 1'.75 from the core of NGC 346. HD 5980 is a well-studied WR/LBV binary system, with some recent observations suggesting it is potentially a quadruple system (Koenigsberger et al. 2014).

\section{Analysis}

\subsection{Atmospheric parameters}

We have employed model-atmosphere grids calculated with the TLUSTY and SYNSPEC codes (Hubeny 1988; Hubeny \& Lanz 1995; Hubeny et al. 1998; Lanz \& Hubeny 2007). They cover a range of effective temperature, $12000 \leq T_{\text {eff }} \leq 35000 \mathrm{~K}$ in steps of typically $1500 \mathrm{~K}$. Logarithmic gravities (in $\mathrm{cm} \mathrm{s}^{-2}$ ) range from 4.5 dex down to the Eddington limit in steps of $0.25 \mathrm{dex}$, and microturbulences are from $0-30 \mathrm{~km} \mathrm{~s}^{-1}$ in steps of $5 \mathrm{~km} \mathrm{~s}^{-1}$. 
Grids have been calculated for a range of metallicities with that for an SMC metallicity used here. As discussed by Ryans et al. (2003) and Dufton et al. (2005), equivalent widths and line profiles interpolated within these grids are in good agreement with those calculated explicitly at the relevant atmospheric parameters. Full details of the grids can be found in Dufton et al. (2005).

The analysis followed similar methods to those used by Hunter et al. (2008a) when analysing the FSMS data and is only briefly summarised here. Where two (or more) ionisation stages of silicon were observed, the effective temperatures were constrained by requiring that each ionisation stage yielded the same estimated silicon abundance. For the B0 to B0.5 spectral types, the He II $\lambda 4542$ and $\lambda 4686$ lines were also observable and yielded estimates that were normally in good agreement with those from the silicon ionisation equilibrium. For spectral types later than B3, the strength of the He I spectrum becomes temperature sensitive and the well observed $\lambda 4026$ line was used.

Effective temperature estimates for the remaining stars have been taken from the effective temperature-spectral type calibration for the SMC of Trundle et al. (2007). We find that this calibration yields estimates that are in satisfactory agreement with those from the silicon and helium lines, with a mean difference of $460 \pm 1220 \mathrm{~K}$. Our sample contained targets with B2.5 V and B3 V types, which lie outside this calibration and for which we have no independent estimates from the silicon and helium lines. The effective temperature scales from Trundle et al. (2007) for the Galaxy and the SMC differ by typically $2500-3500 \mathrm{~K}$ and between the LMC and SMC by $500 \mathrm{~K}$. We have therefore used these differences to estimate effective temperatures for the B2.5 V and B3 V types in the SMC. Finally, the effective temperature for two B2 II objects was taken as the mean of those for B2 I and B2 III. The adopted effective temperature calibration versus spectral type is summarised in Table 3.

In cases where we were unable to assign unique spectral types, we have assigned effective temperatures appropriate to the mid-points of the range. For spectra without luminosity classifications we have assumed that they are near main-sequence (class V) objects. As discussed in Sect. 4.3, the estimated luminosities are generally consistent with such a classification.

Surface gravities were determined by comparing rotationally-broadened theoretical profiles of the $\mathrm{H} \delta$ and $\mathrm{H} \gamma$ lines to those observed. The surface gravity and effective temperature estimates are correlated and an iterative method was adopted to simultaneously determine these parameters. It should be noted that, as discussed by Dunstall et al. (2011), surface gravity estimates for the Be-type stars may be too small due to continuum contamination from a circumstellar disc.

The analyses presented here have implicitly assumed that the targets are single, although a signifiant number of the apparently single targets may be the primary in a multiple system. Additionally we have estimated atmospheric parameters for 36 SB1 and 11 SB2 B-type systems. Garland et al. (2017) considered the consequences of an unseen secondary in their analysis of VFTS B-type binaries. Making the extreme assumption that the secondary had a featureless spectrum, they found that the estimated effective temperatures (from the silicon ionization equilibrium) and the gravities (from fitting hydrogen line profiles) were too small by typically $500-1000 \mathrm{~K}$ and $0.1-0.3$ dex respectively. In reality, the situation will be more complicated as any secondary making a significant flux contribution is also likely to be a near main sequence B-type star but of slightly later spectral type than the primary. If its absorption features were incorporated into those of the primary (as is very likely for the broad hydrogen lines), the consequences could be different to those modelled by
Table 3. Adopted effective temperature-spectral type calibration.

\begin{tabular}{lccr}
\hline \multirow{2}{*}{ Spectral type } & \multicolumn{4}{c}{ Effective temperature } \\
\cline { 2 - 4 } & I & III & V \\
\hline O5 & - & - & $45200(\mathrm{M})$ \\
O6 & - & - & $42970(\mathrm{M})$ \\
O7 & - & - & $40730(\mathrm{M})$ \\
O8 & - & - & $38500(\mathrm{M})$ \\
O9 & - & - & $36265(\mathrm{M})$ \\
O9.5 & - & - & $35150(\mathrm{M})$ \\
B0 & $27200(\mathrm{~T})$ & - & $32000(\mathrm{~T})$ \\
B0.2 & $25750(\mathrm{~T})$ & - & $30800(\mathrm{~T})$ \\
B0.5 & $24300(\mathrm{~T})$ & - & $29650(\mathrm{~T})$ \\
B0.7 & $22850(\mathrm{~T})$ & $25300(\mathrm{~T})$ & $28450(\mathrm{~T})$ \\
B1 & $22350(\mathrm{~T})$ & $23950(\mathrm{~T})$ & $27300(\mathrm{~T})$ \\
B1.5 & $20650(\mathrm{~T})$ & $22550(\mathrm{~T})$ & $26100(\mathrm{~T})$ \\
B2 & $18950(\mathrm{~T})$ & $21200(\mathrm{~T})$ & $24950(\mathrm{~T})$ \\
B2.5 & $17200(\mathrm{~T})$ & $19850(\mathrm{~T})$ & $23900(\mathrm{E})$ \\
B3 & $15500(\mathrm{~T})$ & $18450(\mathrm{~T})$ & $21500(\mathrm{E})$ \\
B5 & $13800(\mathrm{~T})$ & - & - \\
\hline
\end{tabular}

Notes. The calibration for O-type dwarfs was assumed to be linear and was derived from fitting the results from Mokiem et al. (2006, M). The B-type values were primarily from Trundle et al. $(2007, \mathrm{~T})$ and extended by scaling from Galactic and LMC estimates from Trundle et al. (E).

Garland et al. (2017). For example, as the hydrogen line spectrum in B-type stars strengthens as one moves to later spectral types, the gravity estimates could become too large. In summary, the atmospheric parameters for all the targets may contain additional uncertainties due to the presence of secondaries. However the discussion above implies that these are unlikely to be significantly larger than the stochastic uncertainties discussed below.

Twenty eight O-type stars were also observed, which are generally hotter than the models in our TLUSTY grid. Four have been analysed previously by Bouret et al. (2013) for \#1008, \#1012, and \#1071 ${ }^{4}$, and Heap et al. (2006) for \#1019 and their estimates are listed in Table A.2. For the remainder we adopt temperatures using the calibration for O-type dwarfs from the analysis of $~ 30$ O-type stars in the SMC by Mokiem et al. (2006, which included results for 21 of the stars observed in NGC 346 by the FSMS and is summarised in Table 3). Seven of the targets are designated as SB1 and the atmospheric parameters for these (and indeed) other O-type targets may be affected by the presence of unseen secondaries as discussed for the B-type sample. Given the complications of modelling the winds of O-type stars we do not attempt to derive surface gravities or wind parameters for these stars here.

The atmospheric parameters of our sample of stars are given in the Table A.2. For completeness, we also list the parameters from Hunter et al. (2008a) and Mokiem et al. (2006) for the FSMS sample ${ }^{5}$; when no atmospheric parameters were provided, we have followed the same spectral type methodology as for the Survey spectroscopy. Effective temperatures (and gravities)

\footnotetext{
4 Estimates for \#1008 and \#1012 have also been presented by Bouret et al. (2003), Heap et al. (2006), and Massey et al. (2009).

5 Hunter et al. (2008a) did not provide atmospheric parameters for \#0111 although the He II spectrum is clearly present in the FSMS spectroscopy. We have therefore analysed this spectroscopy to obtain atmospheric parameters, although we note that the HST imaging reveals this to be a visual composite of two nearly equal magnitude sources.
} 
could not be estimated for 15 targets. Six were SB2 systems where the spectral type of the primary was uncertain, seven lay beyond our spectral type calibration (five B9 II and two B5e targets), and \#1001 and \#1024 had peculiar spectra (as discussed in Appendix B, respectively). Additionally, no gravity estimate is given for \#1038 (B0:e) as no convincing fit could be obtained for its Balmer line profiles.

Comparison of the effective temperatures derived from the silicon and helium lines for our sample and for B-type samples obtained with the same instrumentation in the LMC (Garland et al. 2017; Dufton et al. 2018) imply that these estimates will have a stochastic uncertainty of typically $\pm 1000 \mathrm{~K}$. The effective temperature estimates deduced from the spectral type calibration will be more uncertain. The comparison with those estimated from the silicon and helium lines discussed above and the scatter in effective temperatures estimates for stars of the same spectral type implies that where the spectral type is well defined a conservative stochastic uncertainty of $\pm 1500 \mathrm{~K}$ is appropriate. For those stars with a range of spectral types or no luminosity classification the uncertainties may be larger. For the surface gravity estimates, the values from the $\mathrm{H} \delta$ and $\mathrm{H} \gamma$ lines generally agreed to within 0.1 dex. Taking into account the uncertainties in the effective temperature estimates, an error of $0.2 \mathrm{dex}$ in the surface gravity would appear to be appropriate.

\subsection{Projected rotational velocities}

Projected rotational velocities have been estimated using two independent methodologies, viz. profile fitting $(\mathrm{PF})$ and Fourier Transform (FT). For the former, the TLuSTY theoretical model at the closest grid point to the parameters given in Table A.2 was adopted. An absorption line profile was then scaled to have the same strength as that observed and the instrumental broadening was included by convolving with a Gaussian profile. The resulting profile was then rotationally broadened assuming a linear limb darkening law with $\epsilon=0.6$ (Gray 2005) until the best fit (by a $\chi^{2}$ minimisation) with the observed profile was found. This method was used previously by Hunter et al. (2008a) where further details can be found.

Metal absorption lines, which are less affected by intrinsic broadening (than for example the hydrogen and diffuse helium lines) provide the most reliable estimates. For our B-type stars we have used either the Mg II $\lambda 4481$ or Si III $\lambda 4552$ lines, whichever was stronger. For stars with significant rotational velocities the metal lines were not well defined, and we used the He I $\lambda 4026$ line. The rotational broadening of our targets was generally larger than the intrinsic broadening, so the choice of theoretical profile was not critical.

For the O-type stars we have adopted theoretical profiles from the grid of Lanz \& Hubeny (2003). The mean surface gravity of the O-type dwarf stars from Mokiem et al. (2006) was $\log g \sim 4.15$ dex and we therefore adopted models from the closest effective temperature grid point with $\log g=4.25$ dex. Our targets have spectral types later than $\mathrm{O} 5$ and hence the $\mathrm{He}$ I $\lambda 4026$ line was visible and was used to estimate projected rotational velocity, thereby maintaining consistency with analysis of the B-type stars. The PF estimates are listed in Table A.2 with those from the original FSMS data being taken directly from Hunter et al. $(2009)^{6}$.

\footnotetext{
6 For nine FSMS targets, where no values were tabulated, PF estimates have been obtained as discussed above.
}

Independent estimates were obtained using the FT methodology (Carroll 1933; Simón-Díaz \& Herrero 2007). This has been widely used for early-type stars (see, for example Dufton et al. 2006b, 2013; Lefever et al. 2007; Markova \& Puls 2008; Simón-Díaz et al. 2010, 2017; Fraser et al. 2010; Simón-Díaz \& Herrero 2014) and relies on the convolution theorem (Gray 2005), viz. that the Fourier transform of convolved functions is proportional to the product of their individual Fourier Transforms. It then identifies the first minimum in the Fourier transform for a spectral line, which is assumed to be the first zero in the Fourier transform of the rotational broadening profile with the other broadening mechanisms exhibiting either no minima or only minima at higher frequencies. Further details on the implementation of this methodology are given by Simón-Díaz \& Herrero (2007) and Dufton et al. (2013). Estimates were obtained for the targets observed here and those in the FSMS and are listed in Table A.2. For the SB2 systems the estimates refer to the primary, which is defined as the star having the strongest absorption spectrum. Values were only measured for SB2 systems where the two spectra were well separated in at least one epoch.

The moderate spectral resolving power of our LR02 and LR03 spectra corresponds to a velocity resolution of approximately $40 \mathrm{~km} \mathrm{~s}^{-1}$. This made the estimation of the projected rotational velocities in the sharpest lined stars unreliable. For the PF methodology, the instrumental broadening dominated the observed profiles, while estimates also became more sensitive to the intrinsic profile adopted. For the FT methodology, the position of the first minima (at relatively high frequencies in the Fourier Transform) became difficult to identify. Hence when projected rotational velocity estimates were less than $40 \mathrm{~km} \mathrm{~s}^{-1}$, they have been assigned to a bin with $0 \leq v_{\mathrm{e}} \sin i \leq 40 \mathrm{~km} \mathrm{~s}^{-1}$. For consistency the same approach has been adopted for the previously published PF estimates for the FSMS data. Sixty one targets fell into this category, with 54 targets having estimates of $\leq 40 \mathrm{~km} \mathrm{~s}^{-1}$ using both methodologies. One target had no FT estimate, while for the remaining six objects the larger (three PF and three FT) estimates ranged from $43-51 \mathrm{~km} \mathrm{~s}^{-1}$ implying that they were consistent within the uncertainties discussed below.

For the 47 O-type stars, PF estimates could be obtained for all targets, apart from \#1010, which has been classified as SB3 with asymmetric profiles; additionally, no convincing minimum was found for \#1030 using the FT methodology. Estimates with $v_{\mathrm{e}} \sin i \geq 40 \mathrm{~km} \mathrm{~s}^{-1}$ were obtained for 36 targets and yielded a mean difference (FT-PF) of $4 \pm 16 \mathrm{~km} \mathrm{~s}^{-1}$ and a mean ratio (FT/PF) of $1.04 \pm 0.11$

For the larger B-type sample of 288 targets, 275 PF estimates were available. For those without measurement: five targets were classified as B9 II and lay beyond the low effective temperature limit of our grid, seven targets were classified as SB2, while no convincing fit could be found for \#1024 (see Appendix B). For the FT methodology, 274 estimates were obtained; again no estimates were obtained for the SB2, B9 II targets and \#1024, while no convincing minima could be found in the SB2 target, \#0035. Estimates with $v_{\mathrm{e}} \sin i \geq 40 \mathrm{~km} \mathrm{~s}^{-1}$ for both methodologies were obtained for $223 \mathrm{~B}$-type targets and yielded a mean difference of $1 \pm 12 \mathrm{~km} \mathrm{~s}^{-1}$ and a mean ratio of $1.01 \pm 0.09$. The statistics for the FSMS (61 targets, $-1 \pm 11 \mathrm{~km} \mathrm{~s}^{-1}, 0.99 \pm 0.07$ ) and Survey (162 targets, $2 \pm 13 \mathrm{~km} \mathrm{~s}^{-1}, 1.02 \pm 0.09$ ) were similar.

These statistics imply that the estimates from the two methodologies are in good agreement. The larger errors for the O-type sample may be due to the intrinsic weakness of the He I spectra and/or the larger macroturbulences that have been inferred for these spectral types (Simón-Díaz \& Herrero 2014; 
Simón-Díaz et al. 2017). We have adopted conservative stochastic uncertainties of $\sim 10 \%$ for the larger estimates and $\pm 10 \mathrm{~km} \mathrm{~s}^{-1}$ for the estimates with $v_{\mathrm{e}} \sin i<100 \mathrm{~km} \mathrm{~s}^{-1}$.

\subsection{Luminosities}

Luminosities have been calculated using the methodology described by Hunter et al. (2007). A uniform reddening of $E(B-V)=0.09$ (Massey et al. 1995) with a reddening law of $A_{\mathrm{V}}=2.72 E(B-V)$ (Bouchet et al. 1985) and a distance modulus of 18.91 dex (Hilditch et al. 2005) were adopted. Bolometric corrections from Vacca et al. (1996) and Balona (1994) were used for stars hotter and cooler than $28000 \mathrm{~K}$, respectively. Luminosity estimates were estimated for all targets apart from the 15 stars without effective temperature estimates (see Sect. 4.1) and are listed in Table A.2. The main sources of uncertainty will arise from the bolometric correction and the extinction. We estimate that these will typically contribute a stochastic error of \pm 0.5 in the absolute magnitude corresponding to \pm 0.2 dex in the luminosity. For stars in the innermost part of the cluster the adopted PSF-fitting photometry might have been influenced by the diffuse light from unresolved fainter stars. This is generally less than $10 \%$ compared to the magnitudes of our sources and less significant than the uncertainties already discussed. Additionally, there may be a systematic error due to the adopted distance to the SMC. Hilditch et al. (2005) estimated an uncertainty in their distance modulus of $\sim 0.1$, which would translate to a systematic error in the luminosity estimates of 0.04 dex.

As discussed in Sect. 4.1, for the 66 targets with no luminosity class, an effective temperature appropriate to a luminosity class V was adopted. Most of these have estimated luminosities consistent with them being close to the main sequence. However 13 targets have luminosities that are more than 0.3 dex larger than this main sequence luminosity (identified by a linear fit between luminosity and effective temperature for all targets with a luminosity class $\mathrm{V}$ designation). Hence the use of a luminosity class $\mathrm{V}$ calibration to estimate the effective temperature may not be appropriate; we have identified these targets in Table A.2 and their physical parameters should be treated with caution.

\subsection{Masses and ages}

We have used BONNSAI ${ }^{7}$ to estimate the evolutionary masses and ages of the stars in our samples. BonNSAI uses a Bayesian methodology and the grids of models from Brott et al. (2011b) to constrain the evolutionary status of a given star, including its age and mass (see Schneider et al. 2014, for details). As independent prior functions, we adopted the SMC metallicity grid of models, a Salpeter (1955) initial mass function, the initial rotational velocity distribution estimated by Hunter et al. (2008a), a random orientiation of spin axes, and a uniform age distribution. The estimates of effective temperature, luminosity and FT projected rotational velocity (taken from Table A.2) were then used to constrain masses. For all targets, the predicted current and initial masses were very similar with differences $<5 \%$ and in Table A.2, we have therefore only listed the current mass estimates.

Using the adopted errors on the effective temperature (see Sect. 4.1), luminosity (Sect. 4.3) and projected rotational velocities (Sect. 4.2), BONNSAI returned $1 \sigma$-uncertainties for all the quantities that it estimates. In the case of the stellar masses, these

\footnotetext{
7 The BonNSAI web-service is available at: www.astro.uni-bonn de/stars/bonnsai.
}

were generally $6-8 \%$ and never greater than $10 \%$. For the ages, the errors were normally 10-15\% (and always less than 20\%) for targets with estimated ages greater than 5 Myr. For younger targets the absolute error in the age estimates was typically 1-2 Myr. The corresponding larger fractional uncertainty reflects the position of the targets close to the zero age main sequence (ZAMS) and indeed for the youngest targets the lower error bound was consistent with the target lying on the ZAMS.

There may be additional uncertainties due to binarity or lineof-sight composites (unresolved at the distance of the SMC). These could affect the effective temperature estimates as discussed in Sect. 4.1 and also lead to an overestimate of the luminosity for the primary. We have investigated the consequences of this by arbitrarily decreasing the luminosity estimates for \#0043 (B0 V) and \#0099 (B2 III) by 0.2 dex (corresponding to a secondary flux contribution of approximately $35 \%$ ); these apparently single stars were chosen as their effective temperature estimates lie at the upper and lower ranges of our B-type sample. Mass estimates were reduced by $\sim 10 \%$ for both stars with the age estimates decreasing by $10 \%$ and increasing by $15 \%$ respectively. Hence even for apparently single stars care must be taken when interpreting these estimates.

For the SB2 target, \#0013, BONNSAI mass estimates can be compared with those deduced from the orbital analysis by Ritchie et al. (2012), The BonNsal estimate in Table A.1 is based on an uncertain spectral type (B1:) for the brighter component and a luminosity estimate that has not been corrected for binarity. As such it is larger than that found for the brighter component by Ritchie et al. $\left(2012,11.9 \pm 0.6 M_{\odot}\right)$. We can also compare mass estimates based on the stellar parameters given by Ritchie et al. (2012) and our estimated system luminosity. For the brighter component, this leads to an effective temperature of $24500 \pm 1500, \log L / L_{\odot}$ of $4.67 \pm 0.2 \mathrm{dex}$ and $v_{\mathrm{e}} \sin i$ of $110 \pm 10 \mathrm{~km} \mathrm{~s}^{-1}$; the values for the secondary component are $34500 \pm 3000 \mathrm{~K}, 4.50 \pm 0.2 \mathrm{dex}$ and $320 \pm 30 \mathrm{~km} \mathrm{~s}^{-1}$ respectively. BONNSAI returns mass estimates of $12.8_{-1.7}^{+1.9}$ and $16.0_{-2.1}^{+2.4} M_{\odot}$. compared with $11.9 \pm 0.6$ and $19.1 \pm 1.0 M_{\odot}$ from Ritchie et al. (2012). Within the error bars, the estimates are in reasonable agreement, and indeed decreasing the adopted luminosity ratio would improve the agreement for both components. This is consistent with the good agreement that Schneider et al. (2014) found in their BONNSAI analysis of Galactic binaries.

Estimates could not be obtained for 22 targets. Fifteen had no effective temperature estimates (see Sect. 4.1) and hence no luminosity estimates as their bolometric corrections were unknown. As such they had insufficient constraints to estimate masses or ages. The remaining seven targets failed the posterior predictive check and/or a $\chi^{2}$-test. These were all classified as supergiants - four B8Ib/II, and three B1.5-B3 spectral types. For the former it was also not possible to find solutions using the effective temperature, gravity and $v_{\mathrm{e}} \sin i$ estimates as constraints, implying that the models of Brott et al. (2011b) did not cover these stellar parameters. For the three remaining targets, solutions could be found using these constraints but implied logarithmic luminosities (in solar units) of 5.83-5.95 dex. These are far higher than those found in Sect. 4.3 and may be related to a mass discrepancy discussed by, for example, Herrero et al. (1992) and Markova et al. (2018). Hence we have not included these mass and age estimates in Table A.2.

In Sect. 4.2, projected rotational velocities were estimated for our sample and in Sect. 5.3.6, these are deconvolved to estimate the rotational velocity distribution. This is similar to that estimated by Hunter et al. (2008a) but shows evidence for a double peaked structure. Previously Schneider et al. (2017, 
Table 4. Estimates of the mass (in units of $M_{\odot}$ ) and age (in Myr) for different choices of the rotational velocity distribution.

\begin{tabular}{|c|c|c|c|c|c|c|c|c|c|}
\hline \multirow[t]{2}{*}{ Star } & \multirow[t]{2}{*}{$T_{\text {eff }}$} & \multirow[t]{2}{*}{$\log L$} & \multirow[t]{2}{*}{$v_{\mathrm{e}} \sin i$} & \multicolumn{2}{|c|}{ H08 } & \multicolumn{2}{|c|}{ Flat } & \multicolumn{2}{|c|}{ VFTS } \\
\hline & & & & Mass & Age & Mass & Age & Mass & Age \\
\hline 0099 & 18000 & 3.79 & 98 & 7.0 & 36.8 & 7.0 & 36.8 & 7.0 & 36.8 \\
\hline 0070 & 30500 & 4.43 & 102 & 13.8 & 7.9 & 13.8 & 7.8 & 13.8 & 7.7 \\
\hline 1088 & 19850 & 4.06 & 184 & 8.6 & 24.7 & 8.8 & 24.7 & 8.6 & 24.7 \\
\hline 1230 & 29650 & 4.06 & 198 & 11.8 & 3.9 & 11.8 & 3.4 & 11.8 & 3.5 \\
\hline 1212 & 21500 & 3.78 & 307 & 8.0 & 24.0 & 8.0 & 24.0 & 8.0 & 24.0 \\
\hline 0079 & 29500 & 4.37 & 308 & 13.4 & 8.0 & 13.4 & 7.8 & 13.4 & 7.9 \\
\hline
\end{tabular}

Notes. Estimates are provided for the adopted distribution of Hunter et al. (2008a) (H08), a flat distribution (Flat) and the distribution found by Dufton et al. (2013) for B-type stars in 30 Doradus (30 Dor) and are given to one decimal place to aid comparisons. Other stellar parameters are taken from Table A.2.

Appendix A) found that the different choices of the rotational velocity distribution could lead to significant differences in the mass and age estimates.

Hence in order to investigate the sensitivity of our estimates to the adopted distribution, we have considered two alternatives - a flat distribution (with any rotational velocity being equally probable) and that found for B-type stars in 30 Doradus, which shows similar evidence for a double peaked structure (see Fig. 7). Targets with projected rotational velocities of approximately 100,200 and $300 \mathrm{~km} \mathrm{~s}^{-1}$ and effective temperatures of approximately 20000 and $30000 \mathrm{~K}$ were analysed with the results summarized in Table 4. In general, different rotational velocity distributions lead to very similar estimates of the stellar mass and luminosity. Indeed in over half the cases, there is no change in the estimates as BonNsAI selected the same evolutionary models. The maximum ranges in mass and age estimates are only $0.2 M_{\odot}$ and $0.5 \mathrm{Myr}$ respectively. As these are both lower than the uncertainties in the estimates discussed above, we conclude that uncertainties due to the choice of rotational velocity distribution are unlikely to be significant at least for targets near the hydrogen burning main sequence.

\section{Discussion}

We now discuss our estimates of the stellar parameters and projected rotational velocities obtained in Sect. 4. We generally adopt our FT estimates of the latter but also consider the PF estimates when they might lead to different conclusions.

\subsection{Stellar masses and ages}

As discussed by Indu \& Subramaniam (2011), the SMC has experienced significant recent star formation with peaks at 0-10 Myr and 50-60 Myr. Unlike the LMC, where this activity has been concentrated in the north and north-eastern regions, recent star formation in the SMC has not been found to have significant spatial structure. NGC 346 as a young association is located in the central part of the brightest SMC H II region, N66 (Henize 1956); its structure has been discussed by, for example, Gouliermis et al. (2008) and Hennekemper et al. (2008).

Deep imaging from the HST has been used by Sabbi et al. (2008) to investigate the spatial variation of the present day mass function in the central part of NGC 346 (with an outer radius of approximately $20 \mathrm{pc}$, equivalent to $\sim 1$ '.13). They found a steeper mass function with increasing radius, implying mass segregation for the most massive stars. The medians of the estimated stellar masses and ages (see Table A.2) for our spectroscopic sample
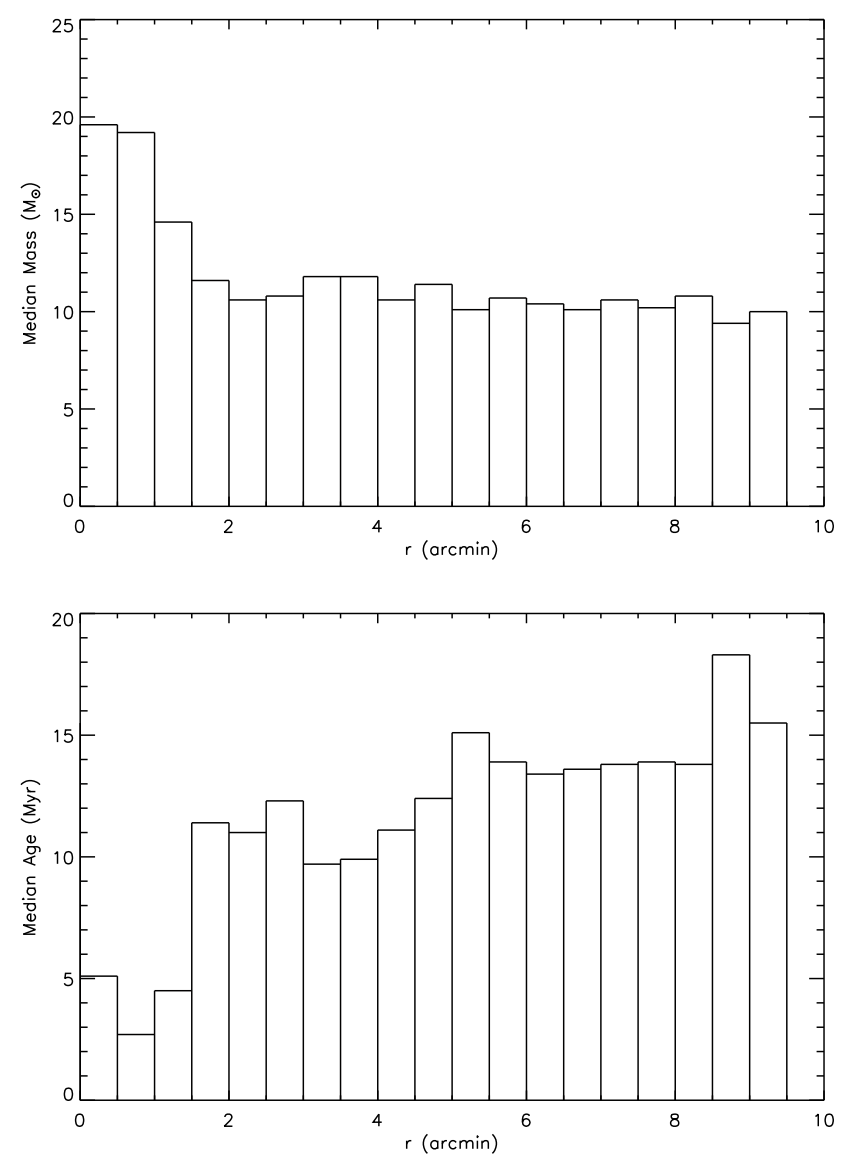

Fig. 3. Median stellar mass (top) and age (bottom) distributions for our NGC 346 spectroscopic sample as a function of radial distance from \#1001.

are shown as a function of radius ( $r$ ) from the centre of NGC 346 in Fig. 3. Our adopted centre was $12^{\prime \prime}$ from that determined by Sabbi et al. (2008), from a consideration of source counts but this will not impact significantly on the discussion below.

Stars with radial distances, $r \lesssim 2$ ! 0 , appear to be more massive, with a relatively flat distribution at larger distances. Additionally, these targets have smaller evolutionary age estimates. Kolmogorov-Smirnov (K-S) tests (Fasano \& Franceschini 1987) for both the estimated masses and ages return very low $(<0.1 \%)$ probabilities that the samples from the inner and outer regions originated from the same parent population. Indeed, the mass distribution suggests that the stars are 

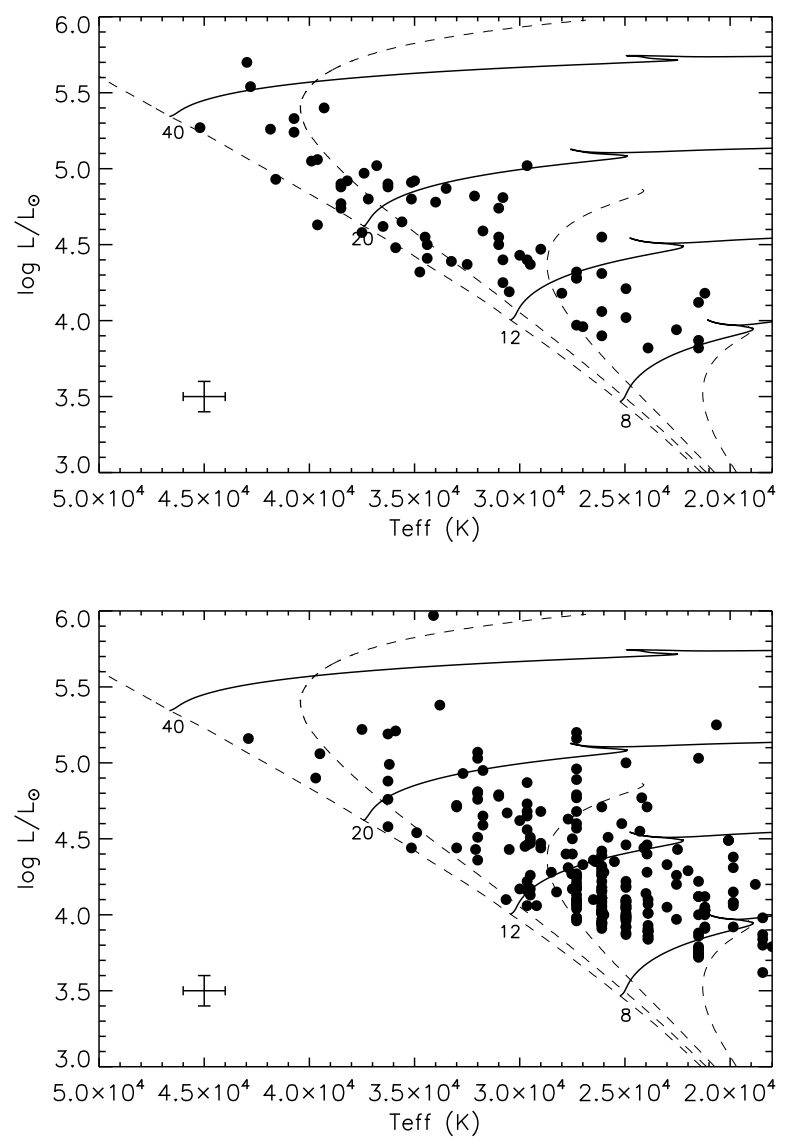

Fig. 4. Hertzsprung-Russell diagrams of the inner ( $r \leq 2$ '.0, upper panel) and outer $(r>2$ '.0, lower panel $)$ regions from our combined NGC 346 sample. Evolutionary tracks are for an initial rotational velocity of approximately $180 \mathrm{~km} \mathrm{~s}^{-1}$. Isochrones (dotted lines) are for, from left-to-right: 0, 3, 10 and $30 \mathrm{Myr}$.

not bound to the central cluster, in agreement with the definition of NGC 346 by Gieles \& Portegies Zwart (2011) as an "association", and reflecting the arguments by Gouliermis et al. (2014) that it is comprised of two distinct components (with an extended distribution of stars that formed hierarchically in addition to the centrally-condensed cluster).

This decrease in the stellar mass and increase in the age estimates with radial distance mirrors the results of Sabbi et al. (2008) for the inner region of NGC 346. It is also consistent with previous studies of the young stellar populations in our Galaxy and the LMC. For the former, Getman et al. (2014a,b) identified similar trends for pre-main sequence (PMS) stars in the Orion molecular cloud complex, whilst recently Getman et al. (2018) found that $80 \%$ of a sample of 19 young clusters exhibited radial age gradients. For the latter, Schneider et al. (2018) deduced ages and masses for approximately 450 apparently single early-type stars in 30 Doradus observed by the VFTS. The medians for the ages and masses as a function of increasing distance from the central R136 star cluster show an increase and decrease respectively, in agreement with our results and those for the Galactic studies. Hence there is increasing evidence that such behaviour may be an ubiquitous feature of massive star formation.

Adopting the threshold of $r<2$ '.0 implied by Fig. 3 (rather than the $r \simeq 3.5$ from Relaño et al. 2002), Hertzsprung-Russell (H-R) diagrams for the inner and outer regions are shown in Fig. 4. Evolutionary tracks and isochrones are from the SMC grid of Brott et al. (2011b) with initial rotational velocities of
$180 \mathrm{~km} \mathrm{~s}^{-1}$, which is consistent with the median NGC 346 projected rotational velocity of $136 \mathrm{~km} \mathrm{~s}^{-1}$ and a random inclination of axes. However for targets near to the main sequence, the evolutionary tracks and isochrones are relatively insensitive to the choice of initial rotational velocity as can be seen from Figs. 5 and 7 of Brott et al. (2011b). Our choice of model grid maintains consistency with the masses and ages estimated using BONNSAI. SMC evolutionary models generated with the Geneva evolutionary code (Georgy et al. 2013a) have qualitatively similar tracks and isochrones but are only available for two initial rotational velocities. Hence we have not tried to use them to estimate masses and ages, although Dufton et al. (2018) found that for VFTS targets with low $v_{\mathrm{e}} \sin i$, the Geneva tracks with zero rotation (Georgy et al. 2013b) yielded masses and ages that were consistent with the BONNSAI estimates within the observational uncertainties.

A significant number of targets lie above the ZAMS and imply a range of stellar ages (cf. the isochrones in Fig. 4) as discussed in Sect. 4.4. Nonetheless, there may be other causes, for example some of these may be either detected (SB1 or SB2) or undetected binaries with the luminosity of the primary being overestimated. For a binary containing targets with a flux ratio of unity, this overestimate would be $0.3 \mathrm{dex}$, which would constitute an upper bound. Inspection of Fig. 4 shows that particularly in the outer region a decrease of even 0.3 dex in the luminosity would not bring most of the targets to the ZAMS. Previous investigations of young Galactic clusters (see, for example, Strom et al. 2005; Dufton et al. 2006a) have also found targets above the main sequence, despite a better discrimination against binarity. Additionally it is possible that some of the targets may be pre-main sequence. However in that case it would be expected that more overluminous stars would be present in the inner region, whilst if anything the reverse would appear to be the case.

Thirteen targets (twelve classified as O-type and one as B0V) have BonNSAI age estimates of less than 1 Myr and $1 \sigma$ upper uncertainties ranging from 0.6 to $2.3 \mathrm{Myr}$. Combining these age estimates and uncertainties to find $1 \sigma$ upper limits on the ages leads to a range of 1.0-2.6 Myr with a median of 1.9 Myr. Hence these massive stars (mainly situated in the inner region) argue for an age of less than $2 \mathrm{Myr}$ for the more recent period of star formation (in agreement with the estimate from Walborn et al. 2000 , based on four stars close to the ZAMS).

However it would appear that even the inner region of NGC 346 is not a simple, coeval stellar population - consistent with VFTS investigations of 30 Doradus (Schneider et al. 2018). Stars in this region (upper panel of Fig. 4) straddle several isochrones and it is clear that the cluster is more complex than just a simple single population (e.g. Cignoni et al. 2011; Gouliermis et al. 2014).

Inspection of the targets with the largest age estimates (30-42 Myr) shows that they are all classified as B3 III. The corresponding dwarf $\mathrm{B} 3 \mathrm{~V}$ population, which would be expected to be younger, lies near the magnitude of the observational cutoff (see Sect. 2) and may not have been well sampled. However Fig. 3 confirms that stars in the outer region of NGC 346 generally appear older. Note that the intermediate-age cluster BS 90 (Bica \& Schmitt 1995) is only a couple of arcminutes to the north of NGC 346, but is sufficiently old (4.5 Gyr, Sabbi et al. 2007; Rochau et al. 2007) that it does not contaminate our spectroscopic sample.

In Fig. 5, the cumulative probability distribution (CPD) is shown for all the NGC 346 age estimates. It is consistent with multiple generations or, effectively, continuous star formation, as discussed by Brott et al. (2011a) for the FLAMES fields 


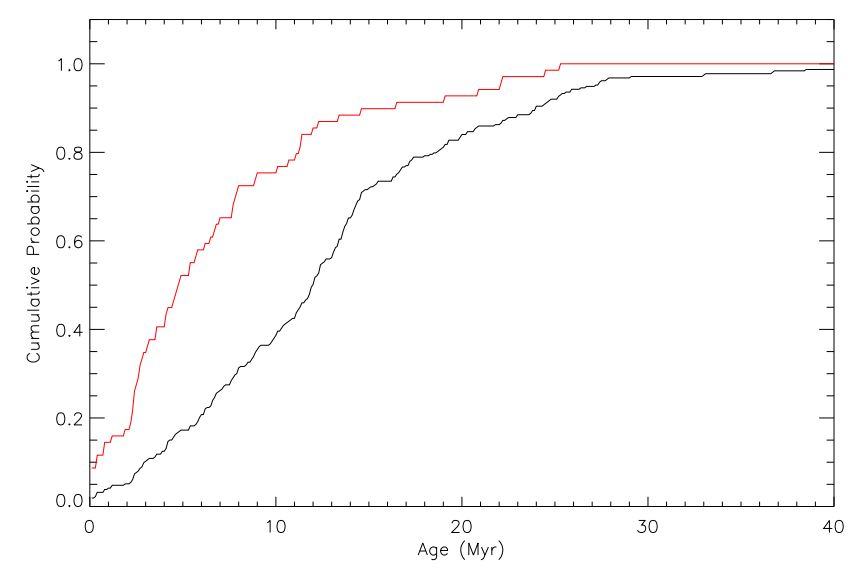

Fig. 5. Cumulative probability function for all 313 targets in NGC 346 with age estimates (solid black line) and for the 69 targets with radial distances of less than $2^{\prime}$ (red line).

observed in the LMC. A histogram of the age distribution shows significant star formation for the last $28 \mathrm{Myr}$ peaking at an age of approximately $16 \mathrm{Myr}$. As discussed above, our sample will be a mixture of cluster members and field stars. We have therefore repeated the above procedures for the 69 targets within a 2 ' radius of our adopted centre of NGC 346. As can be seen in Fig. 5, these targets are (as expected) younger and imply significant star formation in the last $12 \mathrm{Myr}$ peaking in the last $4 \mathrm{Myr}$. As this sample may also contain older field stars, this points to NGC 346 being dominated by a very young stellar population.

\subsection{O-type stars outside the central cluster}

Three of the young O-type targets (\#0046, \#1114 and \#1144) lie outside the inner region at radial distances between 3.8 and 6'.2. We have used the Gaia DR2 data release (Gaia Collaboration $2016,2018)$ to search for pecularities in their proper motions (see Table 5) consistent with them having been formed in the inner region. For a $10^{\prime}$ radius centred on NGC 346, there are approximately 1700 targets with $G \leq 16$ and with proper motion estimates. These will be a combination of foreground and SMC targets but inspection of the proper motion distributions imply that they are heavily weighted to the latter. The median values of this sample are $\mu_{\mathrm{RA}}=0.80 \mathrm{mas} \mathrm{yr}^{-1}$ and $\mu_{\mathrm{Dec}}=-1.22 \mathrm{mas} \mathrm{yr}^{-1}$.

In only one case (the $\mu_{\mathrm{RA}}$ of \#1114) is there a difference from the median value that is significant at a $2 \sigma$-level. This star also has the largest radial distance of the three, lying to the south east of the centre at a separation of $\delta_{\mathrm{RA}}=5.67$ and $\delta_{\mathrm{Dec}}=2.52$. Assuming it was ejected from the centre of NGC 346 soon after birth, its estimated age of $0.4 \mathrm{Myr}$ would lead to a peculiar proper motion of $0.85 \mathrm{mas} \mathrm{yr}^{-1}$. This is substantially larger than its proper motion relative to NGC 346, i.e. $\delta\left(\mu_{\mathrm{RA}}\right)=0.25 \mathrm{mas} \mathrm{yr}^{-1}$. However, the age of this object is relatively poorly constrained with a $1 \sigma$ upper uncertainty of $1.9 \mathrm{Myr}$. Increasing the age to $2.3 \mathrm{Myr}$ would then reduce the required $\delta\left(\mu_{\mathrm{RA}}\right)$ to $0.15 \mathrm{mas} \mathrm{yr}^{-1}$, consistent with the Gaia values. The separation in declination

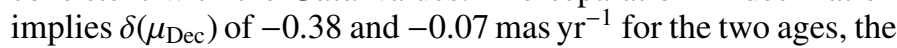
latter being consistent with the effectively zero $\delta\left(\mu_{\text {Dec }}\right)$ implied by the Gaia estimates.

For \#0046, the Gaia estimates imply effectively zero proper motion with respect to NGC 346. The estimated age (1.0 Myr) and upper uncertainty $(0.9 \mathrm{Myr})$ lead to predicted (rel-

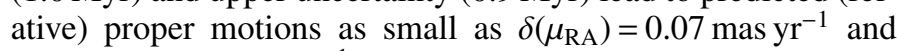
$\delta\left(\mu_{\text {Dec }}\right)=-0.10$ mas $^{-1} r^{-1}$. These are consistent with the Gaia
Table 5. Proper motions (mas $\mathrm{yr}^{-1}$ ) and radial distances $(r)$ from the centre of NGC 346 for selected targets from the DR2 Gaia data release.

\begin{tabular}{llrrrrr}
\hline \hline \multirow{2}{*}{ Target } & \multirow{2}{*}{ ST } & \multicolumn{2}{c}{$r$} & & \multicolumn{2}{c}{ Proper motions (Gaia) } \\
\cline { 3 - 4 } \cline { 6 - 7 } & & $\left({ }^{\prime}\right)$ & \multicolumn{1}{c}{$(\mathrm{pc})$} & & $\mu_{\mathrm{RA}}$ & $\mu_{\text {Dec }}$ \\
\hline 0046 & O7 Vn & 3.8 & 66.9 & & $0.80 \pm 0.08$ & $-1.22 \pm 0.06$ \\
1114 & O9 V & 6.2 & 109.2 & & $1.05 \pm 0.10$ & $-1.22 \pm 0.07$ \\
1144 & O9.5 V & 4.0 & 70.4 & & $1.01 \pm 0.14$ & $-1.25 \pm 0.08$ \\
1134 & B0:e & 2.5 & 44.0 & & $0.82 \pm 0.09$ & $-1.34 \pm 0.06$ \\
1174 & B1-3e & 6.5 & 114.5 & & $0.97 \pm 0.10$ & $-1.25 \pm 0.08$ \\
Median & - & $<10.0$ & $<176.1$ & & 0.80 & -1.22 \\
\hline
\end{tabular}

Notes. Also listed are the median values for targets with $r<10^{\prime}$ from the centre of NGC 346 and with $G \leq 16 \mathrm{mag}$.

estimates within the observational uncertainties, particularly when considering the simple method used to ascertain the motion of NGC 346. Similar arguments apply to the proper motion estimates for \#1144. In summary, the proper motions and age estimates for all three stars are consistent with them having been formed close to the centre of NGC 346, although the uncertainties do not preclude them having been formed elsewhere.

\subsection{Stellar rotational velocities}

\subsubsection{General properties}

Projected rotational estimates have been obtained for effectively all our targets classified earlier than B9 with only seven SB2 systems, the one SB3 system and the peculiar target \#1024 (see Appendix B) having no estimates. The sample is predominantly B-type with 275 estimates compared with 46 O-type values. Additionally there are 21 OB-type supergiants (luminosity classes I-II) with $16 v_{\mathrm{e}} \sin i$ estimates. Significant macroturbulence has been found previously in O-type and early B-type supergiants (Ryans et al. 2002; Simón-Díaz \& Herrero 2014; Simón-Díaz et al. 2017). This would lead to our PF estimates (that include all excess broadening) being larger than the FT estimates (only the rotational component) and indeed a difference of $11 \pm 9 \mathrm{~km} \mathrm{~s}^{-1}$ is found.

The estimates cover a wide range of projected rotational velocities from less than $40 \mathrm{~km} \mathrm{~s}^{-1}$ to approximately $380 \mathrm{~km} \mathrm{~s}^{-1}$. Dividing the sample into $40 \mathrm{~km} \mathrm{~s}^{-1}$ bins, the most populous is that with $0<v_{\mathrm{e}} \sin i \leq 40 \mathrm{~km} \mathrm{~s}^{-1}$, which implies that the sample contains a significant number of slowly rotating stars. This is confirmed by the deconvolution of the projected rotational velocity distribution discussed in Sect. 5.3.6. Additionally there are two very rapidly rotating targets, \#1134 and \#1174, which are discussed further in Sect. 5.3.4.

\subsubsection{Mass variations}

Hunter et al. (2008b) found lower projected rotational velocities for apparently single stars in the FSMS sample with $M>25 M_{\odot}$ compared to those with lower masses in both the Magellanic Clouds and our Galaxy. This was true even in the low metallicity SMC, where the effects of line-driven winds are expected to be weaker (Mokiem et al. 2007), although their high-mass sample was limited to only six stars.

Our sample of non-supergiant targets that have mass estimates $M \geq 25 M_{\odot}$ is over a factor of two larger with 14 objects. 
Again these targets have a lower median ${ }^{8} \mathrm{FT} v_{\mathrm{e}} \sin i$ estimate of $72 \mathrm{~km} \mathrm{~s}^{-1}$ compared with $137 \mathrm{~km} \mathrm{~s}^{-1}$ for the remaining targets; for the PF estimates the medians are 61 and $144 \mathrm{~km} \mathrm{~s}^{-1}$ respectively. $\mathrm{K}-\mathrm{S}$ tests (setting all the upper limits of the $v_{\mathrm{e}} \sin i$ estimates to $40 \mathrm{~km} \mathrm{~s}^{-1}$ ) returned probabilities of $8.4 \%$ (PF estimates) and $12.3 \%$, implying that differences in the projected rotational velocities of the two samples do not have a high level of statistical significance.

Projected rotational velocities have also been determined for both the O-type (Ramírez-Agudelo et al. 2013, 2015) and Btype (Dufton et al. 2013; Garland et al. 2017) stars in the VFTS of 30 Doradus; unfortunately mass estimates are not available for all the targets. However the LMC models of Brott et al. (2011b) imply that $25 M_{\odot}$ models should have a ZAMS effective temperature of $38000-39500 \mathrm{~K}$, depending on the rotational velocity. In turn, the analysis by Ramírez-Agudelo et al. (2017) of the VFTS spectroscopy implies that this should correspond to a spectral type of O6.5 for luminosity class III-V objects. Hence we have repeated the above analysis for the apparently single non-supergiant VFTS targets and find median $v_{\mathrm{e}} \sin i$ estimates of $111 \mathrm{~km} \mathrm{~s}^{-1}$ for the 55 early-O type targets cf. $173 \mathrm{~km} \mathrm{~s}^{-1}$ for the remaining 406 lower-mass objects. Although these are higher than the medians for the NGC 346 targets, they show the same qualitative behaviour. Additionally a $\mathrm{K}-\mathrm{S}$ test returns a very low $(<0.1 \%)$ probability that the projected rotational velocities for two 30 Doradus samples originated from the same parent population.

Further evidence for such a difference is provided by the estimated rotational velocity distributions for the apparently single non-supergiant VFTS O-type (Ramírez-Agudelo et al. 2013) and B-type (Dufton et al. 2013) samples. The former appears unimodal with a mode at approximately $100 \mathrm{~km} \mathrm{~s}^{-1}$ and an extended higher velocity tail, while the latter is bi-modal with maxima at 60 and $300 \mathrm{~km} \mathrm{~s}^{-1}$. In turn this leads to median rotational velocities of $160 \mathrm{~km} \mathrm{~s}^{-1}$ and $250 \mathrm{~km} \mathrm{~s}^{-1}$, respectively. The LMC models of Brott et al. (2011b) imply that the boundary between the two samples will be at a mass of $16 M_{\odot}$. Although this boundary differs from that considered above of $25 M_{\odot}$, the medians again indicate that the more massive stars in general have lower rotational velocities.

In summary there is evidence that in the LMC, targets with masses, $M \geq 25 M_{\odot}$, have lower median projected rotational velocities than less massive early-type stars. A similar difference is seen in our results for the smaller sample in NGC 346, but it is not as statistically significant.

\subsubsection{Spatial variations}

B-type stars in Galactic clusters (see, for example Huang \& Gies 2006; Wolff et al. 2007) appear to rotate more quickly than those in the field, with some evidence in support of this in the LMC (Keller 2004). In Sect. 5.1, targets within 2'.0 of the cluster centre were found to have a higher median mass and a smaller median age than the rest of the sample. We have therefore calculated medians for the $v_{\mathrm{e}} \sin i$ estimates of the apparently single B-type stars (excluding luminosity class I-II supergiants) in these two spatial regions and summarize the results in Table $6^{8}$.

In Table 6, we also list the medians from the FSMS $v_{\mathrm{e}} \sin i$ estimates of Hunter et al. (2008a) towards the SMC cluster, NGC 330 (adopting the same selection criteria as for NGC 346). These were obtained using a PF methodology similar to that 8 It was not possible to calculate means given the significant number
of targets with $v_{\mathrm{e}} \sin i \lesssim 40 \mathrm{~km} \mathrm{~s}^{-1}$.
Table 6. Medians of $v_{\mathrm{e}} \sin i$ estimates of single non-supergiant B-type stars for the inner and outer regions of NGC 346 obtained by the profile fitting (PF) and Fourier transform (FT) methods, together with the number of stars in each sample $(N)$.

\begin{tabular}{lrrr}
\hline \hline \multirow{2}{*}{ Region } & \multirow{2}{*}{$N$} & \multicolumn{2}{c}{ Medians } \\
\cline { 2 - 4 } & & PF & FT \\
\hline NGC 346 $\left(r<22^{\prime} 0\right)$ & 33 & 187 & 195 \\
NGC 346 $\left(r>2{ }^{\prime} 0\right)$ & 187 & 143 & 137 \\
NGC 330 & 72 & 143 & - \\
30 Doradus & 250 & - & 195 \\
NGC 6611 & 24 & 134 & - \\
\hline
\end{tabular}

Notes. Results for NGC 330 and 30 Doradus are median values for the "field-like" samples from Hunter et al. (2008a) and Dufton et al. (2013), respectively. Results for the Galactic cluster NGC 6611 are from Dufton et al. (2006a).

adopted here and provide a predominantly SMC "field-like" sample as the large majority lie well beyond the cluster radius (see Evans et al. 2006). We find that the median projected rotational velocities in the outer region of NGC 346 and in the (predominantly field sample) of NGC 330 are in good agreement. Although both samples are almost certainly not purely field stars (and NGC 330 is older than NGC 346), these results serve to illustrate the slower rotational velocities compared to the inner region of NGC 346 in agreement with the Galactic studies.

The stars at larger radial distances have on average larger ages than those within the inner 2:.0 of the cluster as discussed in Sect. 5.1. Hence the variation in median projected rotational velocity may reflect the stellar rotation decreasing as the stars evolve during their hydrogen core burning phase. Inspection of the SMC models of B-type stars by Brott et al. (2011b) shows that this can occur with rotational velocities decreasing by up to $20 \%$. However this is limited to models with low masses $\left(<12 M_{\odot}\right)$ near to the end of this evolutionary phase. For example, for a gravity, $\log g \sim 3.8 \mathrm{dex}$ (which is less than the median gravity for our B-type sample of $3.95 \mathrm{dex}$ ) changes are less than $5 \%$ with the rotational velocity having increased in some cases. Hence environmental effects such as those discussed by Wolff et al. (2007) could also be important.

However these results must be treated with some caution. $\mathrm{K}-\mathrm{S}$ tests (using the same procedure for $v_{\mathrm{e}} \sin i$ upper limits as in Sect. 5.3.2) for the samples in inner and outer regions of NGC 346 returned $P$-values of $16 \%$ (PF estimates) and $20 \%$ (FT estimates). Tests using samples for the inner region of NGC 346 and NGC 330 also returned high probabilities that they originated from the same populations. Hence we conclude that our SMC results are consistent with the Galactic investigations, although our samples remain too small to have a high level of statistical signifigance.

\subsubsection{The rapidly rotating targets, \#1134 and \#1174}

Two targets, \#1134 and \#1174, have estimates of their projected rotational velocity from the He I line at $4026 \AA$ in excess of $500 \mathrm{~km} \mathrm{~s}^{-1}$ (see Table A.2). As discussed by, for example, Dufton et al. (2011), it is difficult to reliably estimate such large values due to the line profiles becoming very broad and shallow. We have therefore also obtained FT estimates from the other diffuse $\mathrm{He}_{\mathrm{I}}$ lines at 4143, 4387 and $4471 \AA$. These lead to mean 
values of $519 \pm 15$ (\#1134) and $508 \pm 17$ (\#1174) in reasonable agreement with the estimates from the $4026 \AA$ line.

Irrespective of the precise values, it is clear that these stars have significantly greater projected rotational velocities than the rest of the sample. Adopting the effective temperatures, luminosities and masses estimated in Table A.2 and the SMC grid of evolutionary models of Brott et al. (2011b) leads to estimates of the critical velocity of $736 \mathrm{~km} \mathrm{~s}^{-1}$ (\#1134) and $724 \mathrm{~km} \mathrm{~s}^{-1}$ (\#1174), assuming an initial equatorial rotational velocity, $v_{\mathrm{i}} \simeq$ $530 \mathrm{~km} \mathrm{~s}^{-1}$. These critical velocities are relatively insensitive to the initial rotational velocity with a change of less than $10 \mathrm{~km} \mathrm{~s}^{-1}$ for the models with $v_{\mathrm{i}} \simeq 590 \mathrm{~km} \mathrm{~s}^{-1}$. In turn, this implies that both stars are rotating at more than $70 \%$ of the critical velocity. Indeed, given that the average values for $\sin i$ assuming a random distribution of inclination axes is 0.785 (Gray 2005), it is possible that both stars are rotating at near critical velocities.

Dufton et al. (2011) previously discussed an LMC star, VFTS 102 with a spectral type O9: Vnnne and a projected rotational velocity, $v_{\mathrm{e}} \sin i=600 \pm 100 \mathrm{~km} \mathrm{~s}^{-1}$, lying approximately $12 \mathrm{pc}$ from the X-ray pulsar PSR J0537-6910 in the plane of the sky. They suggested that this object originated from a binary system with its high projected rotational velocity resulting from mass transfer from the progenitor of PSR J0537-691. We have therefore searched for pulsars and supernova remnants (SNRs) in the vicinity of \#1134 and \#1174.

The closest pulsar in the ATNF Pulsar Catalogue 9 (Manchester et al. 2005) is PSR J0100-7211, identified by Lamb et al. (2002) as an anomalous X-ray pulsar. Subsequently, Durant \& van Kerkwijk (2005) identified a possible optical counterpart from HST imaging but this was not confirmed from further deep imaging with the HST. Its X-ray properties were discussed by McGarry et al. (2005), who found a characteristic age of $(P / 2 \dot{P})$ of 6800 yr. Both \#1134 and \#1174 lie approximately $0.15^{\circ}$ from the pulsar corresponding to approximately $160 \mathrm{pc}$ at the distance of the SMC. Adopting the characteristic age would then require a relative velocity in excess of $23000 \mathrm{~km} \mathrm{~s}^{-1}$ for them to have previously been physically connected. Hence we conclude that it is highly unlikely that either star were physically associated with the progenitor of PSR J0100-7211. This is confirmed by their proper motion estimates (see Table 5), which are consistent with the median values for NGC 346. Similar arguments would apply to other SMC pulsars, although we note that their beamed emission implies that other such objects may lie closer to our two targets but remain undetected at present.

The nearest catalogued supernova remnant to either target is B0057-72.2 (also designated B0057-72.4). The position found by Filipović et al. (2002) from radio observations leads to angular separations of 3:7 (\#1134) and 5:7 (\#1174), which correspond to sky separations of approximately 65 and $100 \mathrm{pc}$ respectively. However these distances must be treated with caution given the large angular size of the SNR. For example, Davies et al. (1976) from optical imaging found major and minor axes of $14^{\prime}$ and $11^{\prime}$ with evidence for sub-structure.

Given the large rotational velocities combined with the $\mathrm{Be}$ nature of both targets (and nebular contamination in \#1134) it was difficult to recover robust estimates of their radial velocities. Nonetheless, estimates from the $\mathrm{He}_{\mathrm{I}}$ lines are not inconsistent with what we would expect for the region (cf. results from Evans et al. 2006), i.e. they do not appear to be significantly off-

\footnotetext{
9 An updated version of this catalogue is available from: http:// www. atnf.csiro.au/research/pulsar/psrcat
}

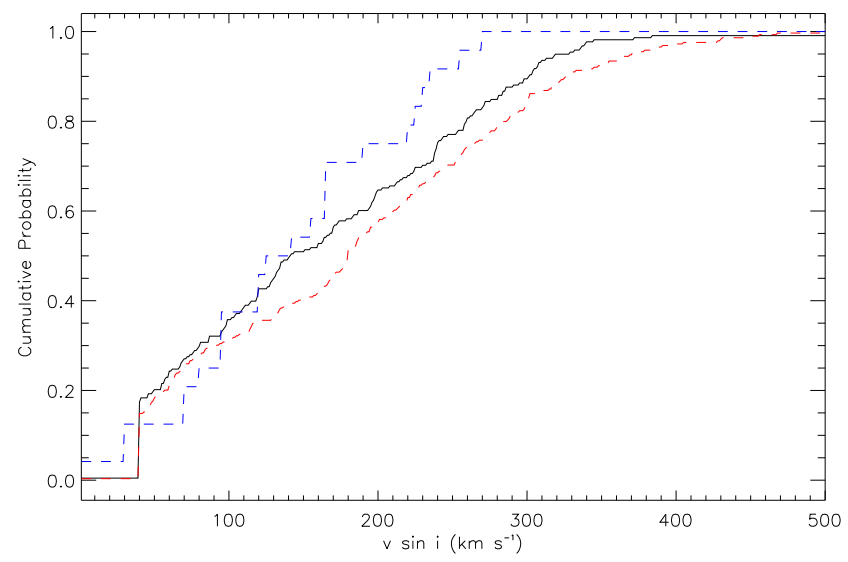

Fig. 6. Cumulative probability distributions for the single B-type stars in NGC 346 (black line), 30 Doradus (red dotted line), NGC 6611 (blue dotted line). Upper limits on $v_{\mathrm{e}} \sin i$ estimates have been set to $40 \mathrm{~km} \mathrm{~s}^{-1}$.

set from the local systemic value, suggesting neither is a significant $(\mathrm{RV})$ runaway.

In summary, there is no direct evidence that either of these targets originated from a binary system where their companion became a supernova. However, we cannot discount that they were originally the secondary components of a binary system. Mass transfer from the primary would then lead to their rapid rotation and their characterisation as a single star as discussed by, for example, de Mink et al. (2013, 2014) and Boubert \& Evans (2018). Although their evolutionary pathways remain unclear, their projected rotational velocities suggest that they may have had a different evolutionary history to the rest of our sample.

\subsubsection{Metallicity effects}

Our projected rotational velocity estimates can be compared with those found from the VFTS to search for effects due to the different metallicities of the Clouds. The VFTS spectroscopy was obtained with same instrumentation to that used here, while the data reduction and analysis methodologies were similar. Therefore, we do not expect any major systematic differences between estimates from the two datasets.

Estimates for the O-type single and binary targets were obtained by Ramírez-Agudelo et al. (2013, 2015), with those for the B-type samples being taken from Dufton et al. (2013) and Garland et al. (2017). We have limited our comparison to samples of hydrogen core burning objects (i.e. excluding luminosity classes I and II). In Fig. 6, we display the CPD for our largest sub-sample of single B-type stars, together with those for the same cohort in 30 Doradus (Dufton et al. 2013).

The distributions are similar although there is some evidence for the B-type stars in 30 Doradus having higher rotational velocities. In part, this may reflect a higher fraction of this sample being associated with clusters (Evans et al. 2011). This would be consistent with the median projected rotational velocity (see Table 6) for 30 Doradus being in good agreement with that for the inner region of NGC 346 but larger than those for the field dominated sample.

We have investigated this further using statistical tests for the four sub-samples of the O- and B-type targets split into apparently single and binary systems. $\mathrm{K}-\mathrm{S}$ and Kuiper tests were performed and the results are summarized in Table 7. For all cases, there is no evidence that the projected rotational velocity distributions in NGC 346 and 30 Doradus are different at a 5\% signif- 
Table 7. Statistical tests on the estimated projected rotational velocities for main-sequence (MS) stars in the NGC 346 (346), VFTS (30 Dor), and NGC 6611 (6611) samples.

\begin{tabular}{|c|c|c|c|c|c|c|c|}
\hline \multirow[t]{2}{*}{ Sample } & \multicolumn{3}{|c|}{ Number } & \multicolumn{2}{|c|}{$\mathrm{K}-\mathrm{S}$} & \multicolumn{2}{|c|}{ Kuiper } \\
\hline & 346 & 30 Dor & 6611 & $\mathrm{D}$ & $\mathrm{P}$ & $\mathrm{D}$ & $\mathrm{P}$ \\
\hline Single MS O-type & 34 & 189 & - & 0.117 & 0.804 & 0.159 & 0.936 \\
\hline Binary MS O-type & 9 & 104 & - & 0.154 & 0.981 & 0.253 & 0.982 \\
\hline Single MS B-type & 218 & 289 & - & 0.119 & 0.055 & 0.133 & 0.173 \\
\hline Binary MS B-type & 40 & 95 & - & 0.203 & 0.175 & 0.272 & 0.178 \\
\hline Single MS B-type & 218 & - & 24 & 0.210 & 0.262 & 0.351 & 0.063 \\
\hline
\end{tabular}

Notes. The first four columns identify the samples and the number of estimates. For the B-type samples, targets classified later than B3 were excluded. The latter four columns list the D-statistic and probability that the two samples come from the same parent population (P) for the Kolmogorov-Smirnov (K-S) and Kuiper tests.

icance level. In turn this implies that there is no evidence for the rotational velocity distributions being different in the two metallicity environments.

For completeness, in Fig. 6 we also show the CPD for the projected rotational velocity estimates of B-type stars in NGC 6611 based on the FSMS observations (Dufton et al. 2006a) and list the median value in Table 6. The CPD implies that the NGC 6611 targets have, on average, lower rotational rates, which is supported by its lower median. As the NGC 6611 targets should be all cluster members, this would be consistent with faster rotation occurring in lower metallicity environments. However the statistical tests summarized in Table 7 show that these differences are not significant at a 5\% level, due to the relatively small sample size for NGC 6611.

\subsubsection{Deconvolution of the $v_{\mathrm{e}} \sin i$ distribution}

Our sample of apparently single non-supergiant B-type targets is large enough to estimate the current distribution of their rotational velocities. Assuming that their rotation axes are randomly distributed, we can infer the probability density function distribution, $P\left(v_{\mathrm{e}}\right)$, using the iterative procedure of Lucy (1974) as implemented by Dufton et al. (2013), where further details can be found. The two extremely fast rotators (\#1134 and \#1174) have been excluded from the deconvolution for numerical stability reasons.

The probability distributions implied by both the FT and PF $v_{\mathrm{e}} \sin i$ estimates are shown in Fig. 7, together with that for the equivalent sample in the 30 Doradus region (Dufton et al. 2013). The two probability distributions inferred for the NGC 346 are broadly similar, reflecting the good agreement between the PF and FT estimates as discussed in Sect. 4.2.

As discussed in Sect. 5.3.5, the CDFs for the $v_{\mathrm{e}} \sin i$ estimates of NGC 346 and 30 Doradus were consistent with them having the same parent population and this must be borne in mind when considering possible differences. In general, the distributions are similar with a significant population of stars having small rotational velocities and with a second maximum at approximately $300 \mathrm{~km} \mathrm{~s}^{-1}$. The NGC 346 sample has some additional structure between 80 and $200 \mathrm{~km} \mathrm{~s}^{-1}$ but given the differences between the two $P\left(v_{\mathrm{e}}\right)$ distributions based on the PF and FT estimates, this cannot be considered significant. Additionally, as discussed by Dufton et al. (2013), the use of a bin size of $40 \mathrm{~km} \mathrm{~s}^{-1}$ in the de-convolution implies that structure on this scale or smaller is unlikely to be real. There may also be differences at high rotational velocities $\left(v_{\mathrm{e}}>350 \mathrm{~km} \mathrm{~s}^{-1}\right)$ but given the relatively small number of targets with large projected rotational velocities (see Fig. 6), these can again not be considered significant.

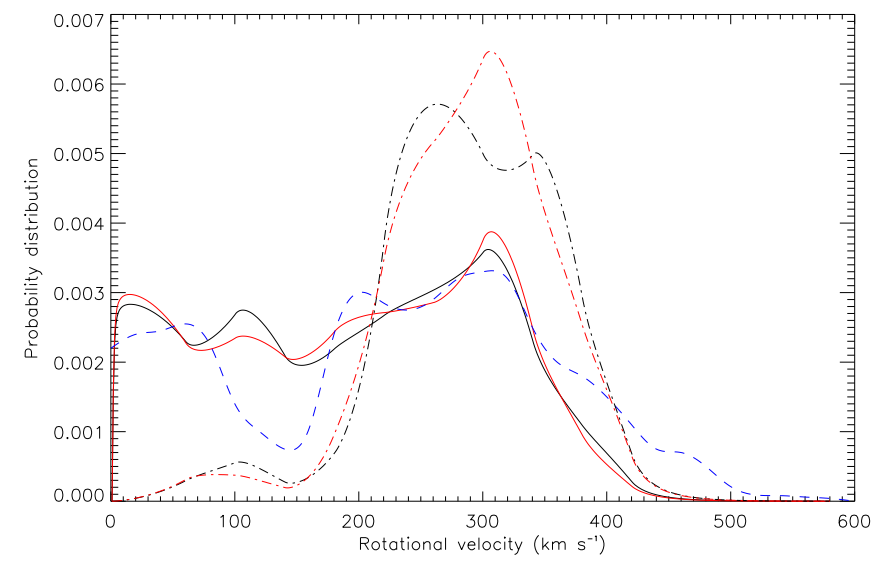

Fig. 7. Rotational velocity probability distributions, $f\left(v_{\mathrm{e}}\right)$, for the apparently single B-type stars in NGC 346 deduced from the PF (black line) and FT (red line) estimates, and for the equivalent sample in 30 Doradus (dotted blue line) of Dufton et al. (2013). Also shown are the equivalent distributions for the apparently single Be-type (dash-dotted black and red lines).

For both regions approximately $10 \%$ of the targets have rotational velocities of less than $40 \mathrm{~km} \mathrm{~s}^{-1}$. For 30 Doradus, Dufton et al. (2018) found that a significant fraction of these targets had enhanced nitrogen abundances that were inconsistent with current single star evolutionary models. They considered possible explanations, of which the most promising would appear to be braking due to magnetic fields (Morel 2012; Aerts et al. 2014) or stellar mergers with subsequent magnetic braking (Schneider et al. 2016). A similar investigation of the Btype stars in NGC 346 would be valuable.

Our best estimates of the probability density function for the apparently single B-type stars (excluding supergiants) are given in Table 8. These values require a judgement about the nature of the smaller scale structure as discussed above. Also listed is the cumulative distribution function corresponding to these estimates and, because this is truncated at $v_{\mathrm{e}} \leq 500 \mathrm{~km} \mathrm{~s}^{-1}$, it does not reach unity. We recommend that either the range up to the critical velocity is populated or that the probability density function is renormalised, depending on the nature of the application.

\subsubsection{Rotational velocities of the Be-type stars}

Classical Be-type stars have prominent emission features in their Balmer line spectrum, indicating the presence of a geometrically flattened, circumstellar disc (Quirrenbach et al. 1994, 
Table 8. Estimates of the probability density of the rotational velocity, $P\left(v_{\mathrm{e}}\right)$, and its cumulative distribution function (cdf) for the NGC 346 apparently single B-type sample.

\begin{tabular}{lcc}
\hline \hline$v_{\mathrm{e}}$ & $P\left(v_{\mathrm{e}}\right) \times 10^{3}$ & cdf \\
\hline 0 & 2.90 & 0.0000 \\
20 & 2.90 & 0.0580 \\
40 & 2.75 & 0.1145 \\
60 & 2.40 & 0.1660 \\
80 & 2.25 & 0.2125 \\
100 & 2.20 & 0.2570 \\
120 & 2.15 & 0.3005 \\
140 & 2.15 & 0.3435 \\
160 & 2.20 & 0.3870 \\
180 & 2.25 & 0.4315 \\
200 & 2.45 & 0.4785 \\
220 & 2.60 & 0.5290 \\
240 & 2.80 & 0.5830 \\
260 & 2.95 & 0.6405 \\
280 & 3.10 & 0.7010 \\
300 & 3.60 & 0.7680 \\
320 & 3.50 & 0.8390 \\
340 & 2.55 & 0.8995 \\
360 & 1.70 & 0.9420 \\
380 & 1.00 & 0.9690 \\
400 & 0.60 & 0.9850 \\
420 & 0.25 & 0.9935 \\
440 & 0.10 & 0.9970 \\
460 & 0.05 & 0.9985 \\
480 & 0.02 & 0.9992 \\
500 & 0.00 & 0.9994 \\
\hline & & \\
\hline
\end{tabular}

Notes. Supergiants (luminosity classes I and II) were excluded from the de-convolution.

1997). Zorec \& Briot (1997) have estimated that approximately $17 \%$ of Galactic stars showed the Be phenomenon, with the highest fraction around B1e-B2e. Emission is also often seen in the helium and iron spectra, with silicon and magnesium emission lines being seen in some stars (Porter \& Rivinius 2003). A recent review of the $\mathrm{Be}$ phenomenum has been given by Rivinius et al. (2013).

Seventy three targets have been identified as Be-type equating to $25 \%$ of the total B-type sample; this increases to $27 \%$ if supergiants are excluded. Nine of the Be-type stars are binaries and excluding these leads to $29 \%$ of the apparently single stars (excluding supergiants) being Be-type. These percentages are higher than those found by Zorec \& Briot (1997, 17\%) for Galactic field stars and by Martayan et al. (2006a, 18\%) towards NGC 2004 in the LMC (this sample was predominantly field stars). Our Be-type percentages are similar to that of Martayan et al. (2007 26\%) for the field towards NGC 330 and Bonanos et al. (2010, 27\%) from an infrared investigations of the SMC. Martayan et al. (2006a, 2007) found tentative evidence that the proportion of Be-type stars in clusters might be higher but these were based on small numbers of B-type stars (LMC: 19 stars, SMC: 18 stars). For our sample of 33 apparently single B-type stars within 2'.0 of the centre of NGC 346, we find 6 Be-type stars (excluding \#1100 which is possibly a Herbig Bstar) leading to a percentage of $18 \%$, with an upper $95 \%$ confidence limit of $33 \%$. Hence although there is no evidence for an increased Be-type fraction in the centre of NGC 346, our sample is too small to exclude that possibility
The mechanisms underlying the Be phenomenon remain unclear but such stars in our Galaxy are believed to be fast rotators with velocities that range from $\sim 60 \%$ up to 100\% (Chauville et al. 2001; Frémat et al. 2005; Cranmer 2005; Rivinius et al. 2006; Ekström et al. 2008) of the critical limit (Townsend et al. 2004). For our sample, this is consistent with the small number of Be-type stars with low $v_{\mathrm{e}} \sin i$ estimates and a correspondingly large median value of $240 \mathrm{~km} \mathrm{~s}^{-1}$ compared with those in Table 6 . For the apparently single Be-type stars, we have performed a de-convolution of the $v_{\mathrm{e}} \sin i$ estimates using the same methodology as summarized in Sect. 5.3.6. In Fig. 7, these are shown for both the PF and FT $v_{\mathrm{e}} \sin i$ estimates. The sample size is small and hence caution should be exercised in interpreting these results. For example, there appears to be a population of slowly rotating Be-type stars $\left(v_{\mathrm{e}} \sim 100 \mathrm{~km} \mathrm{~s}^{-1}\right)$ but this is based on a relatively small number (9-10) of targets with correspondingly low $v_{\mathrm{e}} \sin i$ estimates.

The rotational velocities of our Be-type sample mainly lie in the range $\sim 200-450 \mathrm{~km} \mathrm{~s}^{-1}$. Dunstall et al. (2011) inferred rotational velocity distributions for Be-type samples in the Magellanic Clouds assuming a Gaussian distribution. For the SMC, these peaked at rotational velocities of $260 \mathrm{~km} \mathrm{~s}^{-1}$ and $310 \mathrm{~km} \mathrm{~s}^{-1}$ for the FSMS and the survey of Martayan et al. (2006b) respectively in good agreement with the results found here. Adopting the critical velocity estimates from Sect. 5.3.4 would then imply that they are rotating at $\sim 0.3-0.6$ of this velocity. This is lower than that generally found for Galactic targets but as discussed by Rivinius et al. (2013), there may be systematic biases in our $v_{\mathrm{e}} \sin i$ estimates. These would include additional absorption in shell stars and line emission in $\mathrm{Be}$ stars, as well as the presence (of a presumably narrower lined) secondary.

In summary, the projected rotational velocities of our Betype stars are systematic larger than those of the rest of the B-type stars. This implies rotational velocities between 200$450 \mathrm{~km} \mathrm{~s}^{-1}$, although the Be-type velocities may be subject to systematic biases.

\section{Conclusions}

We have presented spectral types and estimates of the atmospheric parameters and projected rotational velocities for the hot star population towards NGC 346. Additionally, we have inferred masses and ages using the BonNSAI package (Schneider et al. 2014). Our conculsions are:

1. Targets towards the inner region of NGC 346 have higher median masses and smaller ages than the rest of the sample. There appears to be a population of very young targets with ages of less than 2 Myr. These are predominantly in the inner region, while the three young targets at greater radial distances could also have been formed nearer the centre.

2. The more massive targets may have lower median projected rotational velocities consistent with previous studies of the Magellanic Clouds.

3. Targets close to the centre of NGC 346 have a higher mean projected rotational velocities than those at larger radial distances consistent with previous Galactic studies.

4. Two targets (\#1134 and \#1174) have very large projected rotational velocities and are rotating in excess of $70 \%$ of their critical velocities. Their origin is investigated and especially whether they could be the original secondaries in a binary where the primary evolved into a supernova. However it is 
not possible to draw firm conclusions from the current observations.

5. A comparison with the 30 Doradus region in the LMC finds no evidence for significant differences in early-type stellar rotational velocities with metallicity. There is some evidence that the SMC targets rotate faster than those in young Galactic clusters but this is not statistically significant. These results agree with the conclusions from ultraviolet studies of early-type stars in the Clouds (Penny et al. 2004; Penny \& Gies 2009).

6. The projected rotational velocities for the apparently single B-type hydrogen burning targets have been deconvolved to infer their rotational velocity distribution. This shows that a significant number have low rotational velocities $(\simeq 10 \%$ with $v_{\mathrm{e}}<40 \mathrm{~km} \mathrm{~s}^{-1}$ ). Additionally the distribution peaks at a rotational velocity of approximately $300 \mathrm{~km} \mathrm{~s}^{-1}$.

7. Relatively large projected rotational velocity have been found for our Be-type sample and imply rotational velocities between $200-450 \mathrm{~km} \mathrm{~s}^{-1}$. As our Be-type $v_{\mathrm{e}} \sin i$ estimates may be systematically biased to lower values, this difference between the rotational velocities of the B-type and Be-type stars may be even larger.

The results presented here are based on samples of approximately 350 and 750 targets in the SMC and LMC respectively. Further progress in understanding the rotational velocities of early-type stars in the Magellanic Clouds will require significantly larger samples of targets. Additionally it will be important to ensure that the samples are well constrained in both their spatial position (field or cluster) and their physical parameters (binarity, mass, age etc.).

Acknowledgements. Based on observations at the European Southern Observatory in programme 074D.0011 and 171.D-0237. This work has made use of data from the European Space Agency (ESA) mission Gaia (https://www cosmos.esa.int/gaia), processed by the Gaia Data Processing and Analysis Consortium (DPAC, https://www.cosmos.esa.int/web/gaia/dpac/ consortium). Funding for the DPAC has been provided by national institutions, in particular the institutions participating in the Gaia Multilateral Agreement CJE and DJL acknowledge the OWC. CJE thanks Linda Smith for obtaining the AAT spectra, and is grateful for the past hospitality of the Space Telescope Science Institute and travel funding from its Director's Discretionary Research Fund (DDRF).

\section{References}

Aerts, C., Molenberghs, G., Kenward, M. G., \& Neiner, C. 2014, ApJ, 781, 88 Azzopardi, M., \& Vigneau, J. 1975, A\&AS, 19, 271

Azzopardi, M., \& Vigneau, J. 1982, A\&AS, 50, 291

Balona, L. A. 1994, MNRAS, 268, 119

Bica, E. L. D., \& Schmitt, H. R. 1995, ApJS, 101, 41

Blecha,, A., North, P., Royer, F., \& Simond, G. 2003, BLDR Software Reference Manual, 1st edn.

Bonanos, A. Z., Lennon, D. J., Köhlinger, F., et al. 2010, AJ, 140, 416

Boubert, D., \& Evans, N. W. 2018, MNRAS, 477, 5261

Bouchet, P., Lequeux, J., Maurice, E., Prevot, L., \& Prevot-Burnichon, M. L. 1985, A\&A, 149, 330

Bouret, J., Lanz, T., Hillier, D. J., et al. 2003, ApJ, 595, 1182

Bouret, J.-C., Lanz, T., Martins, F., et al. 2013, A\&A, 555, A1

Brott, I., Evans, C. J., Hunter, I., et al. 2011a, A\&A, 530, A116

Brott, I., de Mink, S. E., Cantiello, M., et al. 2011b, A\&A, 530, A115

Carroll, J. A. 1933, MNRAS, 93, 478

Chauville, J., Zorec, J., Ballereau, D., et al. 2001, A\&A, 378, 861

Cignoni, M., Tosi, M., Sabbi, E., Nota, A., \& Gallagher, J. S. 2011, AJ, 141, 31

Cranmer, S. R. 2005, ApJ, 634, 585

Currie, M. J., Berry, D. S., Jenness, T., et al. 2014, Astron. Data Anal. Soft. Syst. XXIII, 485, 391

Davies, R. D., Elliott, K. H., \& Meaburn, J. 1976, MmRAS, 81, 89

de Mink, S. E., Langer, N., Izzard, R. G., Sana, H., \& de Koter, A. 2013, ApJ, 764,166 de Mink, S. E., Sana, H., Langer, N., Izzard, R. G., \& Schneider, F. R. N. 2014, ApJ, 782, 7

Dufton, P. L., Ryans, R. S. I., Trundle, C., et al. 2005, A\&A, 434, 1125

Dufton, P. L., Smartt, S. J., Lee, J. K., et al. 2006a, A\&A, 457, 265

Dufton, P. L., Ryans, R. S. I., Simón-Díaz, S., Trundle, C., \& Lennon, D. J. 2006b, A\&A, 451, 603

Dufton, P. L., Dunstall, P. R., Evans, C. J., et al. 2011, ApJ, 743, L22

Dufton, P. L., Langer, N., Dunstall, P. R., et al. 2013, A\&A, 550, A109

Dufton, P. L., Thompson, A., Crowther, P. A., et al. 2018, A\&A, 615, A101

Dunstall, P. R., Brott, I., Dufton, P. L., et al. 2011, A\&A, 536, A65

Dunstall, P. R., Dufton, P. L., Sana, H., et al. 2015, A\&A, 580, A93

Durant, M., \& van Kerkwijk, M. H. 2005, ApJ, 628, L135

Ekström, S., Meynet, G., Maeder, A., \& Barblan, F. 2008, A\&A, 478, 467

Evans, C. J., \& Howarth, I. D. 2003, MNRAS, 345, 1223

Evans, C. J., Howarth, I. D., Irwin, M. J., Burnley, A. W., \& Harries, T. J. 2004, MNRAS, 353, 601

Evans, C. J., Smartt, S. J., Lee, J.-K., et al. 2005, A\&A, 437, 467

Evans, C. J., Lennon, D. J., Smartt, S. J., \& Trundle, C. 2006, A\&A, 456, 623

Evans, C. J., Taylor, W. D., Hénault-Brunet, V., et al. 2011, A\&A, 530, A108

Fasano, G., \& Franceschini, A. 1987, MNRAS, 225, 155

Filipović, M. D., Bohlsen, T., Reid, W., et al. 2002, MNRAS, 335, 1085

Fraser, M., Dufton, P. L., Hunter, I., \& Ryans, R. S. I. 2010, MNRAS, 404, 1306

Frémat, Y., Zorec, J., Hubert, A.-M., \& Floquet, M. 2005, A\&A, 440, 305

Gaia Collaboration (Prusti, T., et al.) 2016, A\&A, 595, A1

Gaia Collaboration (Brown, A. G. A., et al.) 2018, A\&A, 616, A1

Garland, R., Dufton, P. L., Evans, C. J., et al. 2017, A\&A, 603, A91

Garmany, C. D., Conti, P. S., \& Massey, P. 1987, AJ, 93, 1070

Georgy, C., Ekström, S., Eggenberger, P., et al. 2013a, A\&A, 558, A103

Georgy, C., Ekström, S., Granada, A., et al. 2013b, A\&A, 553, A24

Getman, K. V., Feigelson, E. D., Kuhn, M. A., et al. 2014a, ApJ, 787, 108

Getman, K. V., Feigelson, E. D., \& Kuhn, M. A. 2014b, ApJ, 787, 109

Getman, K. V., Feigelson, E. D., Kuhn, M. A., et al. 2018, MNRAS, 476, 1213

Gieles, M., \& Portegies Zwart, S. F. 2011, MNRAS, 410, L6

Gies, D. R., \& Lambert, D. L. 1992, ApJ, 387, 673

Gouliermis, D. A., Chu, Y.-H., Henning, T., et al. 2008, ApJ, 688, 1050

Gouliermis, D. A., Hony, S., \& Klessen, R. S. 2014, MNRAS, 439, 3775

Gray, D. F. 2005, The Observation and Analysis of Stellar Photospheres (UK: Cambridge University Press)

Grin, N. J., Ramírez-Agudelo, O. H., de Koter, A., et al. 2017, A\&A, 600, A82

Hanuschik, R. W., Hummel, W., Dietle, O., \& Sutorius, E. 1995, A\&A, 300, 163

Harries, T. J., Hilditch, R. W., \& Howarth, I. D. 2003, MNRAS, 339, 157

Heap, S. R., Lanz, T., \& Hubeny, I. 2006, ApJ, 638, 409

Heger, A., \& Langer, N. 2000, ApJ, 544, 1016

Henize, K. G. 1956, ApJS, 2, 315

Hennekemper, E., Gouliermis, D. A., Henning, T., Brandner, W., \& Dolphin, A. E. 2008, ApJ, 672, 914

Herrero, A., Kudritzki, R. P., Vilchez, J. M., et al. 1992, A\&A, 261, 209

Heydari-Malayeri, M., \& Hutsemekers, D. 1991, A\&A, 243, 401

Heydari-Malayeri, M., \& Selier, R. 2010, A\&A, 517, A39

Hilditch, R. W., Howarth, I. D., \& Harries, T. J. 2005, MNRAS, 357, 304

Huang, W., \& Gies, D. R. 2006, ApJ, 648, 580

Hubeny, I. 1988, Comput. Phys. Commun., 52, 103

Hubeny, I., \& Lanz, T. 1995, ApJ, 439, 875

Hubeny, I., Heap, S. R., \& Lanz, T. 1998, in Properties of Hot Luminous Stars, ed. I. Howarth, ASP Conf. Ser., 131, 108

Humphreys, R. M. 1983, ApJ, 265, 176

Hunter, I., Dufton, P. L., Smartt, S. J., et al. 2007, A\&A, 466, 277

Hunter, I., Lennon, D. J., Dufton, P. L., et al. 2008a, A\&A, 479, 541

Hunter, I., Brott, I., Lennon, D. J., et al. 2008b, ApJ, 676, L29

Hunter, I., Brott, I., Langer, N., et al. 2009, A\&A, 496, 841

Indu, G., \& Subramaniam, A. 2011, A\&A, 535, A115

Keller, S. C. 2004, PASA, 21, 310

Keller, S. C., Wood, P. R., \& Bessell, M. S. 1999, A\&AS, 134, 489

Koenigsberger, G., Morrell, N., Hillier, D. J., et al. 2014, AJ, 148, 62

Korn, A. J., Keller, S. C., Kaufer, A., et al. 2002, A\&A, 385, 143

Korn, A. J., Nieva, M. F., Daflon, S., \& Cunha, K. 2005, ApJ, 633, 899

Kudritzki, R. P., \& Puls, J. 2000, ARA\&A, 38, 613

Kudritzki, R. P., Pauldrach, A., \& Puls, J. 1987, A\&A, 173, 293

Lamb, R. C., Fox, D. W., Macomb, D. J., \& Prince, T. A. 2002, ApJ, 574, L29

Lanz, T., \& Hubeny, I. 2003, ApJS, 146, 417

Lanz, T., \& Hubeny, I. 2007, ApJS, 169, 83

Lefever, K., Puls, J., \& Aerts, C. 2007, A\&A, 463, 1093

Lennon, D. J. 1997, A\&A, 317, 871

Lennon, D. J., Dufton, P. L., \& Crowley, C. 2003, A\&A, 398, 455

Lucy, L. B. 1974, AJ, 79, 745

Maeder, A., \& Meynet, G. 2001, A\&A, 373, 555

Maeder, A., Przybilla, N., Nieva, M.-F., et al. 2014, A\&A, 565, A39

Manchester, R. N., Hobbs, G. B., Teoh, A., \& Hobbs, M. 2005, AJ, 129, 1993 
Markova, N., \& Puls, J. 2008, A\&A, 478, 823

Markova, N., Puls, J., \& Langer, N. 2018, A\&A, 613, A12

Martayan, C., Hubert, A. M., Floquet, M., et al. 2006a, A\&A, 445, 931

Martayan, C., Frémat, Y., Hubert, A., et al. 2006b, A\&A, 452, 273

Martayan, C., Floquet, M., Hubert, A. M., et al. 2007, A\&A, 472, 577

Massey, P., Parker, J. W., \& Garmany, C. D. 1989, AJ, 98, 1305

Massey, P., Lang, C. C., Degioia-Eastwood, K., \& Garmany, C. D. 1995, ApJ, 438, 188

Massey, P., Zangari, A. M., Morrell, N. I., et al. 2009, ApJ, 692, 618

Massey, P., Morrell, N. I., Neugent, K. F., et al. 2012, ApJ, 748, 96

McGarry, M. B., Gaensler, B. M., Ransom, S. M., Kaspi, V. M., \& Veljkovik, S. 2005, ApJ, 627, L137

Mennickent, R. E., \& Smith, M. A. 2010, MNRAS, 407, 734

Meynet, G., \& Maeder, A. 2000, A\&A, 361, 101

Meynet, G., \& Maeder, A. 2005, A\&A, 429, 58

Meyssonnier, N., \& Azzopardi, M. 1993, A\&AS, 102, 451

Mokiem, M. R., de Koter, A., Evans, C. J., et al. 2006, A\&A, 456, 1131

Mokiem, M. R., de Koter, A., Vink, J. S., et al. 2007, A\&A, 473, 603

Momany, Y., Vandame, B., Zaggia, S., et al. 2001, A\&A, 379, 436

Morel, T. 2012, in Proceedings of a Scientific Meeting in Honor of Anthony, eds. F. J. Moffat, L. Drissen, C. Robert, N. St-Louis, \& A. F. J. Moffat, ASP Conf. Ser., 465, 54

Niemela, V. S. 2002, in Extragalactic Star Clusters, eds. D. P. Geisler, E. K. Grebel, \& D. Minniti, IAU Symp., 207, 202

Niemela, V., \& Gamen, R. 2004, New A Rev., 48, 727

Niemela, V. S., Marraco, H. G., \& Cabanne, M. L. 1986, PASP, 98, 1133

Pasquini, L., Avila, G., Blecha, A., et al. 2002, The Messenger, 110, 1

Pawlak, M., Graczyk, D., Soszyński, I., et al. 2013, Acta Astron., 63, 323

Penny, L. R., \& Gies, D. R. 2009, ApJ, 700, 844

Penny, L. R., Sprague, A. J., Seago, G., \& Gies, D. R. 2004, ApJ, 617, 1316

Porter, J. M., \& Rivinius, T. 2003, PASP, 115, 1153

Quirrenbach, A., Buscher, D. F., Mozurkewich, D., Hummel, C. A., \& Armstrong, J. T. 1994, A\&A, 283, L13

Quirrenbach, A., Bjorkman, K. S., Bjorkman, J. E., et al. 1997, ApJ, 479, 477

Ramírez-Agudelo, O. H., Simón-Díaz, S., Sana, H., et al. 2013, A\&A, 560, A29

Ramírez-Agudelo, O. H., Sana, H., de Mink, S. E., et al. 2015, A\&A, 580, A92

Ramírez-Agudelo, O. H., Sana, H., de Koter, A., et al. 2017, A\&A, 600, A81

Relaño, M., Peimbert, M., \& Beckman, J. 2002, ApJ, 564, 704

Ritchie, B. W., Stroud, V. E., Evans, C. J., et al. 2012, A\&A, 537, A29

Rivero González, J. G., Puls, J., Najarro, F., \& Brott, I. 2012, A\&A, 537, A79

Rivinius, T., Stefl, S., \& Baade, D. 2006, A\&A, 459, 137

Rivinius, T., Carciofi, A. C., \& Martayan, C. 2013, A\&A Rev., 21, 69

Rochau, B., Gouliermis, D. A., Brandner, W., Dolphin, A. E., \& Henning, T 2007, ApJ, 664, 322
Ryans, R. S. I., Dufton, P. L., Rolleston, W. R. J., et al. 2002, MNRAS, 336, 577

Ryans, R. S. I., Dufton, P. L., Mooney, C. J., et al. 2003, A\&A, 401, 1119

Sabbi, E., Sirianni, M., Nota, A., et al. 2007, AJ, 133, 44

Sabbi, E., Sirianni, M., Nota, A., et al. 2008, AJ, 135, 173

Salpeter, E. E. 1955, ApJ, 121, 161

Sana, H., de Koter, A., de Mink, S. E., et al. 2013, A\&A, 550, A107

Sanduleak, N. 1968, AJ, 73, 246

Schneider, F. R. N., Langer, N., de Koter, A., et al. 2014, A\&A, 570, A66

Schneider, F. R. N., Podsiadlowski, P., Langer, N., Castro, N., \& Fossati, L. 2016, MNRAS, 457, 2355

Schneider, F. R. N., Castro, N., Fossati, L., Langer, N., \& de Koter, A. 2017, A\&A, 598, A60

Schneider, F. R. N., Ramírez-Agudelo, O. H., Tramper, F., et al. 2018, A\&A, 618, A73

Simón-Díaz, S., \& Herrero, A. 2007, A\&A, 468, 1063

Simón-Díaz, S., \& Herrero, A. 2014, A\&A, 562, A135

Simón-Díaz, S., Herrero, A., Uytterhoeven, K., et al. 2010, ApJ, 720, L174

Simón-Díaz, S., Godart, M., Castro, N., et al. 2017, A\&A, 597, A22

Strom, S. E., Wolff, S. C., \& Dror, D. H. A. 2005, AJ, 129, 809

Townsend, R. H. D., Owocki, S. P., \& Howarth, I. D. 2004, MNRAS, 350 , 189

Trundle, C., Dufton, P. L., Hunter, I., et al. 2007, A\&A, 471, 625

Udalski, A., Soszynski, I., Szymanski, M., et al. 1998, Acta Astron., 48, 563

Vacca, W. D., Garmany, C. D., \& Shull, J. M. 1996, ApJ, 460, 914

Venn, K. A. 1999, ApJ, 518, 405

Vink, J. S., \& de Koter, A. 2005, A\&A, 442, 587

Walborn, N. R. 1970, ApJ, 161, L149

Walborn, N. R. 1972, AJ, 77, 312

Walborn, N. R. 1978, ApJ, 224, L133

Walborn, N. R., \& Blades, J. C. 1986, ApJ, 304, L17

Walborn, N. R., Lennon, D. J., Haser, S. M., Kudritzki, R.-P., \& Voels, S. A. 1995, PASP, 107, 104

Walborn, N. R., Lennon, D. J., Heap, S. R., et al. 2000, PASP, 112, 1243

Walborn, N. R., Howarth, I. D., Lennon, D. J., et al. 2002, AJ, 123, 2754

Wisniewski, J. P., Bjorkman, K. S., Magalhães, A. M., et al. 2007, ApJ, 671, 2040

Wolff, S. C., Strom, S. E., Dror, D., \& Venn, K. 2007, AJ, 133, 1092

Wolff, S. C., Strom, S. E., Cunha, K., et al. 2008, AJ, 136, 1049

Woosley, S. E., \& Heger, A. 2006, ApJ, 637, 914

Wyrzykowski, L., Udalski, A., Kubiak, M., et al. 2004, Acta Astron., 54, 1

Yoon, S.-C., \& Langer, N. 2005, A\&A, 443, 643

Zorec, J., \& Briot, D. 1997, A\&A, 318, 443 


\section{Appendix A: Additional tables}

Table A.1. Observational parameters of target stars.

\begin{tabular}{|c|c|c|c|c|c|c|c|}
\hline Star & $\alpha(2000)$ & $\delta(2000)$ & $r_{\mathrm{d}}$ & $V$ & $B-V$ & Spectral Type & Comments \\
\hline 1001 & 005904.49 & -721024.7 & 0.00 & 12.61 & -0.28 & $\mathrm{O} 4 \mathrm{If}+\mathrm{O} 5-6$ & Binary (SB2); WB 1, NMC 26, MPG 435 - O5.5 If; ${ }^{(\dagger)}$ \\
\hline 1002 & 005835.82 & -721624.8 & 6.39 & 12.80 & 0.01 & B1.5 Ia & Sk 73, AzV $210 ;{ }^{(\dagger)} ;$ small $v_{\mathrm{r}}$ shifts of $\sim 10 \mathrm{~km} \mathrm{~s}^{-1}$ \\
\hline 1003 & 005943.46 & -720418.7 & 6.79 & 12.96 & -0.14 & B3 Iab & Sk 81, AzV 234; \\
\hline 1004 & 005705.45 & -720951.9 & 9.13 & 13.34 & 0.01 & A2 Ib & \\
\hline 1005 & 005704.09 & -720857.0 & 9.33 & 13.38 & -0.02 & A2 Ib & \\
\hline 1006 & 005954.88 & -721306.3 & 4.70 & 13.40 & -0.09 & A2 Ib & MPG 859, 2dFS\#5102-B9 (Ib) \\
\hline 1007 & 005854.80 & -721317.1 & 2.97 & 13.46 & 0.03 & B1 Ia & Binary (SB1); AzV 214, MPG 293; \\
\hline 1008 & 005900.75 & -721028.1 & 0.29 & 13.52 & -0.22 & $\mathrm{O} 2 \mathrm{III}\left(\mathrm{f}^{*}\right)$ & WB 3 , NMC 29, MPG $355-O 3 V\left(\left(f^{*}\right)\right) ;$ \\
\hline 1009 & 005810.00 & -721101.8 & 4.22 & 13.59 & -0.06 & B1e & MA93\#1061, KWB346\#34 \\
\hline 1010 & 005900.05 & -721037.8 & 0.40 & 13.66 & -0.26 & O5-6 V((f)) & Binary (SB3); WB 4, NMC 30, MPG $342-O 5.5 V+n e b ;^{(\dagger)}$ \\
\hline 1011 & 005955.49 & -721337.7 & 5.06 & 13.77 & -0.22 & O9 III & Binary (SB1); AzV 238; $(\dagger)$ \\
\hline 1012 & 005901.81 & -721031.1 & 0.23 & 14.21 & -0.25 & O6: V((f)) & Binary (SB1); NMC 28, MPG 368; $(\dagger)$ \\
\hline 1013 & 005906.34 & -720956.1 & 0.50 & 14.28 & -0.15 & $\mathrm{~B} 0.5 \mathrm{e}$ & NMC 37, MPG $482-B 0.5: V+n e b$, KWB346\#85 \\
\hline 1014 & 005957.13 & -720752.5 & 4.76 & 14.39 & -0.14 & B3 Ib + mid A? & Binary (SB2?) \\
\hline 1015 & 005902.92 & -721034.8 & 0.21 & 14.44 & -0.24 & $\mathrm{O} 7 \mathrm{~V}$ & MPG 396-O7 V \\
\hline 1016 & 005936.51 & -721022.6 & 2.45 & 14.45 & -0.08 & B8 Ib & MPG 802 \\
\hline 1017 & 010016.07 & -721244.0 & 5.95 & 14.45 & -0.26 & O9 V & Binary (SB2); OGLE209964 (EB) \\
\hline 1018 & 005700.71 & -720811.3 & 9.73 & 14.48 & -0.11 & B0 ne (shell) & AzV 182, 2dFS\#1187-BO (III); \\
\hline 1019 & 005909.93 & -720548.2 & 4.63 & 14.48 & -0.30 & O6.5f?p & AzV 220, MA93\#1121, KWB346\#89; $\left.{ }^{\dagger}\right)$ \\
\hline 1020 & 005906.86 & -721635.9 & 6.19 & 14.51 & -0.19 & B1 III & Binary (SB1); AzV 219, 2dFS\#1363-B1-3 (II) \\
\hline 1021 & 005941.40 & -720810.4 & 3.60 & 14.63 & -0.21 & B2 II & \\
\hline 1022 & 005755.22 & -721731.1 & 8.87 & 14.63 & -0.06 & B2 II & \\
\hline 1023 & 005858.24 & -721346.5 & 3.40 & 14.64 & -0.13 & B0 V & Binary (SB1); OGLE110243 (EB) \\
\hline 1024 & 005916.61 & -721210.0 & 1.99 & 14.64 & 0.05 & $\mathrm{~A} I+\mathrm{B} ?$ & MPG 665, MA93\#1138, KWB346\#93, OGLE160677 (EB) \\
\hline 1025 & 005912.68 & -721109.1 & 0.97 & 14.66 & -0.21 & O7 V & MPG $615-O 8 \mathrm{~V}$ \\
\hline 1026 & 005912.30 & -721107.9 & 0.94 & 14.70 & -0.21 & $06.5 \mathrm{~V}((\mathrm{f}))$ & MPG 602 \\
\hline 1027 & 005817.40 & -721856.2 & 9.26 & 14.72 & -0.06 & B0-3e & MA93\#1066 \\
\hline 1028 & 005802.99 & -721633.8 & 7.75 & 14.89 & -0.16 & B2.5 III & \\
\hline 1029 & 005947.87 & -720450.8 & 6.48 & 14.89 & -0.24 & B2.5 III & \\
\hline 1030 & 005915.51 & -721111.7 & 1.15 & 14.90 & -0.26 & OC5-6 Vz & MPG 655 - O6 V, KWB346\#459; (†) \\
\hline 1031 & 005842.72 & -721245.8 & 2.88 & 14.96 & -0.12 & B2-3e & MPG 184, MA93\#1090, KWB346\#171 \\
\hline 1032 & 005743.93 & -720432.5 & 8.51 & 14.99 & -0.11 & B0.5: e & \\
\hline 1033 & 005906.01 & -721044.9 & 0.36 & 15.02 & -0.25 & B $0 \mathrm{~V}$ & MPG $471-B 0 V+n e b$ \\
\hline 1034 & 005912.81 & -721052.3 & 0.79 & 15.02 & -0.21 & O9.5-B0 V & NMC 11, MPG $617-09.5 \mathrm{~V}$ \\
\hline 1035 & 005903.97 & -721051.1 & 0.44 & 15.04 & -0.26 & $07.5 \mathrm{~V}((\mathrm{f}))$ & MPG 417 \\
\hline 1036 & 005842.75 & -721428.2 & 4.39 & 15.05 & -0.18 & B2.5 III-II & $2 \mathrm{dFS} \# 1333-B 2(I I I)$ \\
\hline 1037 & 005813.00 & -721535.3 & 6.51 & 15.06 & -0.21 & B0 III & $2 \mathrm{dFS} \# 1295-B 0.5(I V)$ \\
\hline 1038 & 005850.25 & -721713.4 & 6.90 & 15.06 & -0.01 & B0: e & MA93\#1104 \\
\hline 1039 & 005901.89 & -721043.2 & 0.37 & 15.06 & -0.23 & $09.5 \mathrm{~V}$ & Binary (SB1); MPG $370-09.5 \mathrm{~V}$ \\
\hline 1040 & 005749.27 & -721655.3 & 8.69 & 15.07 & -0.19 & Early-B+Early-B & Binary (SB2); OGLE52815 (EB) \\
\hline 1041 & 005819.34 & -721756.6 & 8.29 & 15.07 & -0.33 & B2 III-II & \\
\hline 1042 & 005928.75 & -721016.6 & 1.86 & 15.12 & -0.08 & B1-2e & MPG 771 - B0 V+neb, MA93\#1148, KWB346\#248 \\
\hline 1043 & 005928.85 & -721231.7 & 2.82 & 15.14 & -0.25 & $\mathrm{~B} 0 \mathrm{~V}$ & MPG 770 \\
\hline 1044 & 005831.58 & -721821.0 & 8.33 & 15.15 & -0.23 & B1 III & $2 \mathrm{dFS} \# 1320-B 1-3(I I I)$ \\
\hline 1045 & 005907.33 & -721025.3 & 0.22 & 15.17 & -0.28 & O9 V & Binary (SB1); NMC 24, MPG $495-O 8 \mathrm{~V}$ \\
\hline 1046 & 005818.60 & -720426.9 & 6.92 & 15.20 & -0.27 & B $0.5 \mathrm{~V}$ & \\
\hline 1047 & 005751.15 & -720610.2 & 7.04 & 15.21 & 0.06 & A5 III & $2 \mathrm{dFS} \# 1267-A 3 I I$ \\
\hline 1048 & 005858.79 & -721051.3 & 0.62 & 15.21 & -0.23 & O9 V & MPG $330-O 7.5 \mathrm{~V}$ \\
\hline 1049 & 005858.88 & -721038.8 & 0.49 & 15.21 & -0.26 & $\mathrm{~B} 0 \mathrm{~V}$ & Binary (SB2); NMC 31, MPG $332-B 0 V:+n e b$ \\
\hline
\end{tabular}

Notes. Cross-references to previous identifications are given in the final column as follows: Sanduleak (1968, Sk), Azzopardi \& Vigneau; Azzopardi \& Vigneau (1975; 1982, AzV), Walborn \& Blades (1986, WB), Niemela et al. (1986, NMC), Massey et al. (1989 MPG), Meyssonnier \& Azzopardi (1993, MA93), Keller et al. (1999, KWB), and Evans et al. (2004, 2dFS). Additionally eclipsing binaries (EB) from the OGLE project (Udalski et al. 1998) have been identified. Radial distances from \#1001 ( $r_{\mathrm{d}}$, in arcmin) are given for each star ( $1^{\prime}$ corresponds to a physical distance of $\sim 17.5 \mathrm{pc})$. ${ }^{(\dagger)}$ Further notes on individual stars: 1001: Walborn (1978), Walborn \& Blades (1986): O4 III(n)(f). 1002: Humphreys (1983): B3 I; Lennon (1997): B1.5 Ia. 1003: Humphreys (1983): B3 I. 1007: Massey et al. (1995): B1 III. 1008: Walborn \& Blades (1986): O3 III (f*); Niemela et al. (1986): O3 III(f); Walborn et al. (2000): ON3 III(f*); Walborn et al. (2002): O2 III(f*). 1010: Walborn \& Blades (1986), Niemela et al. (1986): O5-6 V; Massey et al. (2009, but noted as triple system): O5.5 III((f)). 1011: Garmany et al. (1987): O9 III; Walborn et al. (1995): O9 III. 1012: Niemela et al. (1986): O5-6: V; Walborn et al. (2000): O4-5 V((f)). 1018: Garmany et al. (1987): B0n. 1019: Classification adopted from Walborn et al. (2000), also classified as O7 If by Garmany et al. (1987). 1030: Heydari-Malayeri \& Selier (2010): O5 V + OB. 1052: Heydari-Malayeri \& Selier (2010): O9.5 V. 1060: Heydari-Malayeri \& Selier (2010): O8 V. 1079: Heydari-Malayeri \& Selier (2010): B0-0.5 V. 1103: EIS photometry has a non-physical (negative) colour, adopted photometry is from Massey et al. (1989). 1190: MA93\#1169 is a blend of this and another star. 
Table A.1. continued.

\begin{tabular}{|c|c|c|c|c|c|c|c|}
\hline Star & $\alpha(2000)$ & $\delta(2000)$ & $r_{\mathrm{d}}$ & $V$ & $B-V$ & Spectral Type & Comments \\
\hline 1050 & 005845.69 & -721716.6 & 7.02 & 15.22 & -0.13 & B1.5 II & 2dFS\#1337-B1-2 (III) \\
\hline 1051 & 010013.61 & -721244.6 & 5.78 & 15.22 & -0.30 & $\mathrm{O} 9 \mathrm{~V}$ & \\
\hline 1052 & 005905.44 & -721042.3 & 0.30 & 15.27 & -0.22 & B $0 \mathrm{~V}$ & MPG 455; (†) \\
\hline 1053 & 005741.17 & -721120.0 & 6.44 & 15.28 & -0.19 & B0.5 III & $2 \mathrm{dFS} \# 1252-B 1-3(I I I)$ \\
\hline 1054 & 010006.44 & -720924.4 & 4.85 & 15.29 & -0.29 & B1 III & Binary (SB1) \\
\hline 1055 & 005909.82 & -721059.0 & 0.70 & 15.32 & -0.26 & $09.5 \mathrm{~V}$ & NMC 13, MPG $549-O 8 \mathrm{~V}$ \\
\hline 1056 & 005727.79 & -720944.9 & 7.43 & 15.33 & -0.06 & A0 II & \\
\hline 1057 & 005920.28 & -721425.0 & 4.18 & 15.34 & -0.17 & B0 V & \\
\hline 1058 & 005907.63 & -721048.3 & 0.46 & 15.35 & -0.26 & O8: $\mathrm{Vn}$ & NMC 16, MPG 500-O6 V:+neb \\
\hline 1059 & 005931.47 & -720800.0 & 3.17 & 15.36 & -0.24 & B0 V & Binary (SB1) \\
\hline 1060 & 005904.79 & -721102.9 & 0.64 & 15.39 & -0.15 & $08 \mathrm{~V}$ & Binary (SB1); NMC 19, MPG $445-07.5 \mathrm{~V}$; (†) \\
\hline 1061 & 005747.68 & -721718.0 & 9.06 & 15.39 & -0.21 & $\mathrm{~B} 0 \mathrm{~V}$ & $2 \mathrm{dFS} \# 1263-09.5 \mathrm{Ib}$ \\
\hline 1062 & 010007.25 & -720936.9 & 4.87 & 15.45 & -0.27 & B1.5 V (shell) & In-filling in wings of Balmer lines \\
\hline 1063 & 005745.20 & -720806.4 & 6.49 & 15.48 & -0.09 & B5e & MA93\#1032 \\
\hline 1064 & 005818.87 & -721833.2 & 8.86 & 15.50 & -0.24 & B1.5 III & Binary (SB1) \\
\hline 1065 & 005840.55 & -721106.4 & 1.96 & 15.52 & 0.02 & A0 II & NMC 46, MPG 167 \\
\hline 1066 & 005942.71 & -721421.9 & 4.92 & 15.53 & -0.16 & B1-2e & KWB346\#259 \\
\hline 1067 & 005822.90 & -721751.4 & 8.10 & 15.53 & -0.24 & $\mathrm{O} 9 \mathrm{~V}$ & \\
\hline 1068 & 005831.75 & -721549.6 & 5.97 & 15.54 & -0.15 & B0.7 III & 2dFS\#1321-B0-5 (III) \\
\hline 1069 & 005821.20 & -721746.8 & 8.08 & 15.54 & -0.23 & B0: + B0.5: & Binary (SB2) \\
\hline 1070 & 005920.60 & -720958.0 & 1.31 & 15.54 & -0.25 & B2 III & MPG 701 \\
\hline 1071 & 005900.96 & -721109.2 & 0.79 & 15.54 & -0.25 & $06.5 \mathrm{Vz}$ & NMC 34, MPG $356-O 6.5 V+n e b$ \\
\hline 1072 & 005739.89 & -720601.3 & 7.82 & 15.55 & -0.26 & B $0.2 \mathrm{~V}$ & $2 \mathrm{dFS} \# 1247-B 1-5(I I)$ \\
\hline 1073 & 005904.57 & -721037.7 & 0.22 & 15.55 & -0.27 & B0 V & MPG 439 \\
\hline 1074 & 005911.27 & -721001.3 & 0.65 & 15.59 & -0.21 & B $0.5 \mathrm{~V}$ & MPG 583; str. H II \\
\hline 1075 & 010011.82 & -720951.3 & 5.18 & 15.60 & -0.25 & B1 III & \\
\hline 1076 & 005814.95 & -721636.4 & 7.26 & 15.62 & -0.18 & B1 III & \\
\hline 1077 & 010017.42 & -720738.6 & 6.23 & 15.64 & -0.29 & B2.5 III & \\
\hline 1078 & 005811.57 & -721428.2 & 5.73 & 15.64 & -0.26 & B0.5 III & 2dFS\#1292; blended in WFI image with 1093 \\
\hline 1079 & 005911.90 & -721055.8 & 0.77 & 15.64 & -0.24 & B0 III & NMC 12, MPG $595-B 0 \mathrm{~V}{ }^{(\dagger)}$ \\
\hline 1080 & 005918.29 & -720421.0 & 6.15 & 15.65 & -0.28 & B0.5 III & \\
\hline 1081 & 005803.37 & -721629.0 & 7.67 & 15.65 & -0.14 & B1 III & \\
\hline 1082 & 010016.71 & -721434.0 & 6.92 & 15.65 & -0.31 & B0 III & \\
\hline 1083 & 005917.42 & -721347.3 & 3.52 & 15.66 & -0.15 & B3 III & \\
\hline 1084 & 010021.93 & -720844.1 & 6.16 & 15.66 & -0.30 & B1.5 III & \\
\hline 1085 & 005720.52 & -721021.1 & 7.96 & 15.68 & -0.17 & B3 III & \\
\hline 1086 & 005910.45 & -721047.0 & 0.59 & 15.68 & -0.22 & $\mathrm{O} 8 \mathrm{~V}$ & MPG 561 \\
\hline 1087 & 005907.33 & -721321.2 & 2.95 & 15.69 & -0.18 & B2 III & \\
\hline 1088 & 010014.44 & -720900.6 & 5.53 & 15.69 & -0.35 & B2.5 III & \\
\hline 1089 & 005801.71 & -721748.5 & 8.82 & 15.73 & -0.12 & B3e & \\
\hline 1090 & 005906.58 & -721030.5 & 0.19 & 15.73 & -0.09 & B0-3 V & MPG 485; str. H II \\
\hline 1091 & 010016.65 & -721320.1 & 6.25 & 15.74 & -0.30 & B0.5: + B0.5: & Binary (SB2) \\
\hline 1092 & 005910.83 & -721338.3 & 3.26 & 15.75 & -0.22 & B1 V & \\
\hline 1093 & 005811.42 & -721429.2 & 5.75 & 15.75 & -0.26 & B1 V & 2dFS\#1292; blended in WFI image with 1078 \\
\hline 1094 & 005915.87 & -721110.7 & 1.16 & 15.76 & -0.17 & $\mathrm{O} 8 \mathrm{~V}$ & MPG 658; KWB346\#459; str. H II \\
\hline 1095 & 005730.32 & -721558.4 & 9.10 & 15.76 & -0.24 & B3e & \\
\hline 1096 & 005842.47 & -721113.9 & 1.87 & 15.77 & -0.11 & $\mathrm{~B} 0.5 \mathrm{~V}$ & MPG 183 \\
\hline 1097 & 005824.42 & -720532.5 & 5.75 & 15.79 & -0.25 & B0.5: + early B & Binary (SB2) \\
\hline 1098 & 005852.19 & -721719.2 & 6.97 & 15.79 & -0.16 & B $1.5 \mathrm{~V}$ & \\
\hline 1099 & 005801.06 & -721829.7 & 9.43 & 15.80 & 0.07 & A7 III & \\
\hline 1100 & 005902.06 & -721102.4 & 0.66 & 15.80 & -0.03 & B1: (Herbig?) & NMC 33, MPG 374, KWB346\#191; str. H II \\
\hline 1101 & 005914.98 & -721135.2 & 1.42 & 15.82 & -0.21 & B0.5: & Binary (SB1); NMC 1, MPG 644 \\
\hline 1102 & 005936.95 & -721702.9 & 7.09 & 15.83 & -0.15 & B2 (shell) & 2dFS\#1406-B1-3 (III) \\
\hline 1103 & 005834.36 & -720903.9 & 2.67 & 15.32 & 0.80 & F8 I & MPG $125 ;$ \\
\hline 1104 & 005759.60 & -720922.6 & 5.07 & 15.90 & -0.15 & B5 II & \\
\hline 1105 & 005926.81 & -721131.7 & 2.04 & 15.91 & -0.17 & B1-3 V & MPG 757 \\
\hline 1106 & 005745.60 & -720642.3 & 7.08 & 15.91 & 0.05 & A5 III & \\
\hline 1107 & 005910.28 & -721042.7 & 0.53 & 15.91 & -0.29 & $09.5 \mathrm{~V}$ & MPG 557 \\
\hline 1108 & 005707.28 & -721050.1 & 8.98 & 15.93 & -0.24 & B2 III & \\
\hline 1109 & 005757.02 & -721606.8 & 7.69 & 15.94 & -0.21 & B1.5 III & \\
\hline 1110 & 010002.06 & -721003.6 & 4.42 & 15.95 & -0.16 & B1-3e & MA93\#1178, KWB346\#543 \\
\hline 1111 & 005945.44 & -721141.6 & 3.39 & 15.97 & 0.07 & A5 III & MPG 820 \\
\hline 1112 & 005702.96 & -720759.7 & 9.61 & 15.98 & -0.27 & B0 V & $2 \mathrm{dFS} \# 1188-B 0(V)$ \\
\hline 1113 & 005753.34 & -721447.3 & 6.99 & 15.98 & -0.17 & B3 III & \\
\hline 1114 & 010018.26 & -720751.8 & 6.19 & 15.98 & -0.37 & O9 V & \\
\hline 1115 & 010010.87 & -720706.6 & 6.06 & 15.99 & -0.18 & Early Be & \\
\hline
\end{tabular}


Table A.1. continued.

\begin{tabular}{|c|c|c|c|c|c|c|c|}
\hline Star & $\alpha(2000)$ & $\delta(2000)$ & $r_{\mathrm{d}}$ & $V$ & $B-V$ & Spectral Type & Comments \\
\hline 1116 & 005825.10 & -721909.8 & 9.26 & 16.00 & -0.21 & Early B+Early B & Binary (SB2); OGLE104222 (EB) \\
\hline 1117 & 005954.15 & -720647.3 & 5.25 & 16.00 & -0.25 & B2 III & \\
\hline 1118 & 005738.80 & -720655.2 & 7.43 & 16.02 & -0.06 & $\mathrm{~B} 2.5 \mathrm{e}$ & \\
\hline 1119 & 005952.37 & -720802.7 & 4.36 & 16.03 & -0.21 & B1 V & \\
\hline 1120 & 005737.52 & -721729.8 & 9.72 & 16.03 & -0.07 & B8 II & $2 \mathrm{dFS} \# 1243-B 8(I I)$ \\
\hline 1121 & 005811.12 & -721547.1 & 6.75 & 16.03 & -0.02 & A0 II & \\
\hline 1122 & 005950.10 & -721441.1 & 5.52 & 16.04 & -0.12 & B2.5 III & Binary (SB1) \\
\hline 1123 & 005730.23 & -721340.2 & 7.92 & 16.04 & -0.19 & B3e (shell) & \\
\hline 1124 & 005837.43 & -721330.7 & 3.73 & 16.04 & -0.27 & B3 III & \\
\hline 1125 & 005832.20 & -721438.6 & 4.90 & 16.05 & -0.25 & B0 V & \\
\hline 1126 & 005930.99 & -720912.4 & 2.36 & 16.07 & -0.26 & $\mathrm{~B} 1 \mathrm{~V}$ & MPG 786 \\
\hline 1127 & 005912.39 & -721617.6 & 5.91 & 16.09 & -0.08 & $\mathrm{~B} 2-3 \mathrm{e}$ & \\
\hline 1128 & 005908.97 & -721110.4 & 0.84 & 16.11 & 0.04 & $\mathrm{O} 7.5 \mathrm{~V}((\mathrm{f}))$ & MPG 529 \\
\hline 1129 & 005932.81 & -720804.4 & 3.19 & 16.13 & -0.17 & $\mathrm{~B} 1.5 \mathrm{~V}$ & \\
\hline 1130 & 005910.84 & -721717.7 & 6.90 & 16.14 & -0.15 & B1-3e & Binary (SB1); MA93\#1122, \\
\hline 1131 & 010017.21 & -720744.2 & 6.17 & 16.14 & -0.24 & B3 III & \\
\hline 1132 & 005942.54 & -721607.2 & 6.41 & 16.15 & -0.22 & B1 V & \\
\hline 1133 & 005807.01 & -721624.3 & 7.44 & 16.15 & -0.19 & B1 V & \\
\hline 1134 & 005844.91 & -720826.4 & 2.48 & 16.16 & -0.02 & B0:e & MA93\#1097, KWB346\#374 \\
\hline 1135 & 005803.36 & -721050.4 & 4.70 & 16.17 & -0.20 & B1 V & \\
\hline 1136 & 005837.89 & -721628.8 & 6.40 & 16.18 & -0.07 & B1-3e & \\
\hline 1137 & 005958.00 & -721615.9 & 7.14 & 16.18 & -0.20 & B2 V & \\
\hline 1138 & 005724.40 & -721547.9 & 9.37 & 16.20 & -0.16 & B1-3e & MA93\#1004 \\
\hline 1139 & 005914.06 & -720752.1 & 2.65 & 16.20 & -0.02 & A2 II & \\
\hline 1140 & 005917.01 & -721055.5 & 1.09 & 16.20 & -0.22 & B $0 \mathrm{~V}$ & MPG 670 \\
\hline 1141 & 005720.13 & -721115.2 & 8.03 & 16.21 & -0.23 & B2 III & \\
\hline 1142 & 005929.16 & -720839.4 & 2.58 & 16.21 & -0.18 & B1.5 III & \\
\hline 1143 & 005936.89 & -721443.8 & 4.98 & 16.22 & -0.20 & B1.5 V & \\
\hline 1144 & 005911.66 & -721424.7 & 4.04 & 16.23 & -0.16 & $09.5 \mathrm{~V}$ & 2dFS\#1369-O9 V \\
\hline 1145 & 005950.32 & -721449.0 & 5.63 & 16.24 & -0.20 & $\mathrm{~B} 2.5 \mathrm{~V}$ & \\
\hline 1146 & 005809.62 & -721527.7 & 6.57 & 16.24 & -0.09 & B1-3e & MA93\#1059, KWB346\#309 \\
\hline 1147 & 005734.21 & -721240.8 & 7.27 & 16.25 & -0.12 & B1-3e & MA93\#1016 \\
\hline 1148 & 010001.02 & -721525.7 & 6.62 & 16.27 & -0.13 & B1-3e & MA93\#1176 \\
\hline 1149 & 005906.82 & -721719.6 & 6.92 & 16.27 & -0.16 & $\mathrm{~B} 2.5 \mathrm{~V}$ & \\
\hline 1150 & 005827.84 & -721608.0 & 6.37 & 16.27 & -0.19 & B1 V & \\
\hline 1151 & 005954.91 & -721104.0 & 3.91 & 16.27 & -0.14 & B1-2e & MPG 860, MA93\#1171, KWB346\#529 \\
\hline 1152 & 005712.23 & -721309.3 & 9.02 & 16.28 & -0.05 & B9 II & \\
\hline 1153 & 005749.14 & -721330.9 & 6.55 & 16.29 & -0.15 & B2 V & \\
\hline 1154 & 005754.86 & -720719.4 & 6.16 & 16.29 & -0.22 & $\mathrm{~B} 1.5 \mathrm{~V}$ & $2 \mathrm{dFS} \# 1273-B 0-5(I V)$ \\
\hline 1155 & 005844.28 & -721025.8 & 1.55 & 16.30 & -0.21 & B1.5 III & MPG 200 \\
\hline 1156 & 005943.56 & -720448.8 & 6.35 & 16.30 & -0.16 & B $1-3 e$ & MA93\#1161 \\
\hline 1157 & 005926.06 & -720557.2 & 4.75 & 16.30 & -0.09 & A0 III-II & \\
\hline 1158 & 005828.25 & -721101.2 & 2.84 & 16.32 & -0.20 & B3 Ve & MPG 87 \\
\hline 1159 & 005904.05 & -720946.2 & 0.64 & 16.34 & -0.26 & $\mathrm{~B} 1.5 \mathrm{~V}$ & MPG 422 \\
\hline 1160 & 005922.48 & -720737.1 & 3.11 & 16.34 & -0.19 & B1 V & \\
\hline 1161 & 005919.78 & -720956.5 & 1.26 & 16.34 & -0.22 & B2 V & MPG 694 \\
\hline 1162 & 005944.25 & -720907.3 & 3.31 & 16.34 & -0.09 & A0 II & MPG 815 \\
\hline 1163 & 005950.52 & -721028.9 & 3.52 & 16.35 & -0.25 & $\mathrm{~B} 1 \mathrm{~V}$ & MPG 842 \\
\hline 1164 & 005840.14 & -721025.0 & 1.86 & 16.35 & -0.16 & B3 V & MPG 163 \\
\hline 1165 & 010022.65 & -721405.6 & 7.02 & 16.35 & -0.32 & $\mathrm{~B} 1.5 \mathrm{~V}$ & \\
\hline 1166 & 005910.85 & -721805.8 & 7.70 & 16.36 & -0.24 & B1-3e & \\
\hline 1167 & 005939.66 & -721057.3 & 2.75 & 16.37 & -0.22 & B $1.5 \mathrm{~V}$ & MPG 806 \\
\hline 1168 & 005748.10 & -720820.0 & 6.20 & 16.38 & -0.06 & B9 II & \\
\hline 1169 & 005713.64 & -721523.0 & 9.83 & 16.38 & -0.08 & B9 II & \\
\hline 1170 & 005940.75 & -720752.2 & 3.76 & 16.38 & -0.27 & B $0.5 \mathrm{~V}$ & \\
\hline 1171 & 005941.69 & -720347.0 & 7.21 & 16.38 & 0.00 & A2 III-II & \\
\hline 1172 & 005756.61 & -721558.8 & 7.62 & 16.39 & -0.14 & B3 V & Binary (SB1) \\
\hline 1173 & 005820.39 & -721254.8 & 4.20 & 16.39 & -0.22 & $\mathrm{~B} 1 \mathrm{~V}$ & \\
\hline 1174 & 005941.00 & -720434.9 & 6.47 & 16.40 & -0.11 & B1-3e & MA93\#1160 \\
\hline 1175 & 005720.27 & -721256.1 & 8.37 & 16.41 & -0.19 & $\mathrm{~B} 2 \mathrm{~V}$ & \\
\hline 1176 & 005915.90 & -721738.7 & 7.29 & 16.41 & -0.24 & B $1.5 \mathrm{~V}$ & \\
\hline 1177 & 005951.54 & -721620.2 & 6.93 & 16.42 & -0.11 & B1-3e & MA93\#1166 \\
\hline 1178 & 005739.47 & -721408.5 & 7.50 & 16.42 & -0.20 & $\mathrm{~B} 1.5 \mathrm{~V}$ & \\
\hline 1179 & 005806.95 & -720858.3 & 4.63 & 16.42 & -0.23 & $\mathrm{~B} 1.5 \mathrm{~V}$ & \\
\hline 1180 & 010016.11 & -721606.0 & 7.90 & 16.43 & -0.31 & B $0.5 \mathrm{~V}$ & \\
\hline 1181 & 005857.14 & -721225.0 & 2.08 & 16.43 & -0.05 & $\mathrm{~B} 1 \mathrm{~V}$ & Binary (SB1); MPG 318 \\
\hline
\end{tabular}


Table A.1. continued.

\begin{tabular}{|c|c|c|c|c|c|c|c|}
\hline Star & $\alpha(2000)$ & $\delta(2000)$ & $r_{\mathrm{d}}$ & $V$ & $B-V$ & Spectral Type & Comments \\
\hline 1182 & 010010.51 & -721420.5 & 6.40 & 16.43 & -0.40 & B $0.5 \mathrm{~V}$ & Binary (SB1) \\
\hline 1183 & 005729.92 & -720459.8 & 9.04 & 16.44 & -0.19 & B $2.5 \mathrm{~V}$ & \\
\hline 1184 & 010009.54 & -721220.0 & 5.34 & 16.44 & -0.26 & B1-3 V & Asymmetric He lines; OGLE210052 (EB) \\
\hline 1185 & 005912.90 & -720950.1 & 0.86 & 16.44 & -0.24 & B0 V & NGC 57, MPG 621; str. nebular spectrum \\
\hline 1186 & 005943.67 & -720737.6 & 4.09 & 16.45 & -0.22 & $\mathrm{~B} 2 \mathrm{~V}$ & \\
\hline 1187 & 005827.46 & -720942.8 & 2.92 & 16.45 & -0.09 & B5e & MPG 85 \\
\hline 1188 & 005929.40 & -720754.6 & 3.15 & 16.46 & -0.09 & B1-2 V & \\
\hline 1189 & 005928.97 & -721123.4 & 2.11 & 16.46 & -0.25 & B1 V & MPG 772 \\
\hline 1190 & 005952.93 & -720631.1 & 5.38 & 16.46 & -0.05 & B1-3e & KWB346\#526; (†) \\
\hline 1191 & 005829.75 & -720906.9 & 2.96 & 16.46 & -0.20 & $\mathrm{~B} 1.5 \mathrm{~V}$ & Binary (SB1); MPG 99 \\
\hline 1192 & 005812.29 & -721743.7 & 8.34 & 16.46 & -0.18 & $\mathrm{~B} 2 \mathrm{~V}$ & Binary (SB1) \\
\hline 1193 & 005745.09 & -721503.4 & 7.65 & 16.47 & -0.09 & B1-2e & MA93\#1031 \\
\hline 1194 & 005904.61 & -721219.5 & 1.91 & 16.47 & -0.12 & B3e & MPG 431, KWB346\#807 \\
\hline 1195 & 005918.85 & -721351.8 & 3.62 & 16.48 & -0.21 & $\mathrm{~B} 1.5 \mathrm{~V}$ & \\
\hline 1196 & 005912.61 & -721724.4 & 7.02 & 16.48 & -0.18 & B1 V & Binary (SB1) \\
\hline 1197 & 005936.37 & -721248.2 & 3.42 & 16.48 & -0.21 & $\mathrm{~B} 1.5 \mathrm{~V}$ & MPG 800 \\
\hline 1198 & 005938.60 & -721401.7 & 4.46 & 16.49 & -0.22 & B $1.5 \mathrm{~V}$ & 2dFS\#1407 - BO-5 (IV) \\
\hline 1199 & 005853.07 & -721003.2 & 0.94 & 16.49 & -0.21 & $\mathrm{~B} 0 \mathrm{~V}$ & MPG 279, KWB346\#751 \\
\hline 1200 & 005753.09 & -721421.7 & 6.74 & 16.51 & -0.19 & B1 V & \\
\hline 1201 & 005812.35 & -721012.2 & 4.00 & 16.51 & -0.17 & $\mathrm{~B} 2.5 \mathrm{~V}$ & MPG 2 \\
\hline 1202 & 005730.78 & -720744.9 & 7.65 & 16.51 & -0.10 & A0 II & \\
\hline 1203 & 005709.73 & -721033.1 & 8.78 & 16.52 & -0.17 & B2-3e (shell) & \\
\hline 1204 & 005931.81 & -721213.8 & 2.77 & 16.53 & 0.06 & A3 III & MPG 788 \\
\hline 1205 & 005959.94 & -721707.4 & 7.94 & 16.55 & -0.16 & $\mathrm{~B} 2.5 \mathrm{~V}$ & \\
\hline 1206 & 005821.44 & -721228.1 & 3.88 & 16.55 & -0.17 & $\mathrm{~B} 2.5 \mathrm{~V}$ & MPG 46 \\
\hline 1207 & 005757.19 & -721237.0 & 5.60 & 16.56 & -0.17 & B3 V & \\
\hline 1208 & 005738.29 & -721252.2 & 7.04 & 16.56 & -0.21 & $\mathrm{~B} 2.5 \mathrm{~V}$ & \\
\hline 1209 & 005943.43 & -721539.6 & 6.04 & 16.57 & -0.10 & B3 V & Binary (SB1) \\
\hline 1210 & 005930.31 & -721657.1 & 6.83 & 16.57 & -0.14 & B3e (shell) & MA93\#1150 \\
\hline 1211 & 005900.08 & -720552.6 & 4.55 & 16.57 & -0.24 & $\mathrm{~B} 1.5 \mathrm{~V}$ & \\
\hline 1212 & 005904.42 & -720706.5 & 3.30 & 16.58 & -0.16 & B3e & KWB346\#814 \\
\hline 1213 & 005757.53 & -721459.2 & 6.87 & 16.58 & -0.05 & B1-3e & MA93\#1047, KWB346\#575 \\
\hline 1214 & 005909.67 & -720527.8 & 4.96 & 16.59 & -0.11 & B1-3e & MA93\#1120, KWB346\#856 \\
\hline 1215 & 005736.72 & -721512.1 & 8.25 & 16.60 & -0.16 & B3 III & \\
\hline 1216 & 005914.67 & -720826.0 & 2.13 & 16.61 & -0.26 & $\mathrm{~B} 1.5 \mathrm{~V}$ & \\
\hline 1217 & 005944.79 & -721526.7 & 5.90 & 16.62 & -0.18 & $\mathrm{~B} 2.5 \mathrm{~V}$ & \\
\hline 1218 & 005941.81 & -721605.1 & 6.35 & 16.62 & -0.14 & $\mathrm{~B} 2.5 \mathrm{~V}$ & \\
\hline 1219 & 010003.31 & -720939.1 & 4.57 & 16.62 & -0.28 & $\mathrm{~B} 1.5 \mathrm{~V}$ & \\
\hline 1220 & 005844.61 & -720843.3 & 2.27 & 16.63 & -0.24 & $\mathrm{~B} 1.5 \mathrm{~V}$ & \\
\hline 1221 & 005907.34 & -721312.8 & 2.81 & 16.63 & -0.17 & B $0.5 \mathrm{~V}$ & \\
\hline 1222 & 005926.95 & -721359.3 & 3.97 & 16.64 & -0.26 & $\mathrm{~B} 1 \mathrm{~V}$ & \\
\hline 1223 & 010019.50 & -721258.2 & 6.29 & 16.64 & -0.32 & $\mathrm{~B} 1 \mathrm{~V}$ & \\
\hline 1224 & 005749.04 & -720544.2 & 7.43 & 16.64 & -0.09 & B1-2e & \\
\hline 1225 & 005847.83 & -721606.6 & 5.84 & 16.65 & -0.17 & B3 V & \\
\hline 1226 & 010011.92 & -721433.9 & 6.62 & 16.65 & -0.22 & $\mathrm{~B} 1.5 \mathrm{~V}$ & \\
\hline 1227 & 005716.74 & -721238.9 & 8.54 & 16.67 & -0.19 & B3 V & \\
\hline 1228 & 005822.55 & -721858.5 & 9.15 & 16.67 & -0.05 & B9 II & \\
\hline 1229 & 005936.11 & -721432.1 & 4.78 & 16.67 & -0.20 & $\mathrm{~B} 1.5 \mathrm{~V}$ & \\
\hline 1230 & 005818.60 & -721141.0 & 3.74 & 16.67 & -0.27 & B $0.5 \mathrm{~V}$ & MPG 33 \\
\hline 1231 & 005911.79 & -721236.1 & 2.26 & 16.67 & -0.09 & B8 II & MPG 588 \\
\hline 1232 & 005857.30 & -721028.7 & 0.55 & 16.67 & -0.25 & B1 V & MPG 323 \\
\hline 1233 & 005831.45 & -721224.6 & 3.22 & 16.68 & -0.25 & B $0.2 \mathrm{~V}$ & MPG 111 \\
\hline 1234 & 005858.98 & -721318.0 & 2.92 & 16.68 & -0.13 & $\mathrm{~B} 2.5 \mathrm{~V}$ & \\
\hline 1235 & 005800.11 & -721245.7 & 5.46 & 16.69 & -0.17 & B3 V & \\
\hline 1236 & 005910.02 & -721130.3 & 1.17 & 16.69 & -0.22 & $\mathrm{~B} 1 \mathrm{~V}$ & MPG 550 \\
\hline 1237 & 005817.19 & -720234.4 & 8.63 & 16.69 & -0.17 & B3e & \\
\hline 1238 & 010014.48 & -721206.7 & 5.62 & 16.69 & -0.30 & B2.5 V & \\
\hline 1239 & 005815.57 & -721645.6 & 7.37 & 16.70 & -0.17 & $\mathrm{~B} 2 \mathrm{~V}$ & \\
\hline 1240 & 005801.84 & -721447.3 & 6.49 & 16.70 & -0.18 & B3 V & \\
\hline 1241 & 005930.45 & -721135.7 & 2.31 & 16.71 & -0.10 & $\mathrm{~B} 1 \mathrm{~V}$ & Binary (SB1); MPG 781 \\
\hline 1242 & 010013.23 & -720718.5 & 6.11 & 16.71 & -0.24 & B2 V & \\
\hline 1243 & 005858.00 & -720625.1 & 4.02 & 16.72 & -0.15 & B3 V & \\
\hline 1244 & 005921.70 & -721110.9 & 1.53 & 16.73 & -0.21 & $\mathrm{~B} 2.5 \mathrm{~V}$ & MPG 710 \\
\hline 1245 & 005756.91 & -721412.0 & 6.41 & 16.73 & -0.19 & $\mathrm{~B} 1.5 \mathrm{~V}$ & \\
\hline 1246 & 005954.80 & -721526.3 & 6.33 & 16.74 & -0.18 & B3 V & Binary (SB1); OGLE207517 (EB) \\
\hline 1247 & 005908.87 & -721036.4 & 0.39 & 16.74 & -0.24 & $\mathrm{~B} 1.5 \mathrm{~V}$ & MPG 527 \\
\hline
\end{tabular}


Table A.2. Atmospheric parameters $\left(T_{\mathrm{eff}}, \log g\right)$ and projected rotational velocities $\left(v_{\mathrm{e}} \sin i\right)$, luminosities, current masses and ages for the NGC 346 sample.

\begin{tabular}{|c|c|c|c|c|c|c|c|c|c|c|c|}
\hline \multirow[t]{2}{*}{ Star } & \multirow[t]{2}{*}{ Spectral Type } & \multirow{2}{*}{$\begin{array}{l}T_{\text {eff }} \\
(\mathrm{K})\end{array}$} & \multirow[t]{2}{*}{ Method } & \multirow{2}{*}{$\begin{array}{l}\log g \\
(\mathrm{dex})\end{array}$} & \multicolumn{2}{|c|}{$v_{\mathrm{e}} \sin i$} & \multirow{2}{*}{$\begin{array}{c}r \\
\left({ }^{\prime}\right) \\
\end{array}$} & \multirow{2}{*}{$\begin{array}{r}\log \mathrm{L} \\
\left(L_{\odot}\right) \\
\end{array}$} & \multirow{2}{*}{$\begin{array}{l}\text { Mass } \\
\left(M_{\odot}\right)\end{array}$} & \multirow{2}{*}{$\begin{array}{r}\text { Age } \\
\text { (Myr) }\end{array}$} & \multirow[t]{2}{*}{ Comment } \\
\hline & & & & & & $\begin{array}{r}\text { FT } \\
-1)\end{array}$ & & & & & \\
\hline 0001 & O7 Iaf+ & 34100 & M06 & 3.35 & 74 & 76 & 2.13 & 5.97 & 55.6 & 2.9 & SB1 \\
\hline 0002 & A2: Iab & - & - & - & - & - & 5.55 & - & - & - & \\
\hline 0003 & G0: & - & - & - & - & - & 3.36 & - & - & - & \\
\hline $0004^{*}$ & Be (B1:) & 27300 & $\mathrm{AM}$ & 3.00 & 266 & 258 & 7.22 & 5.16 & 22.4 & 6.6 & \\
\hline 0005 & A0 II & - & - & - & - & - & 8.49 & - & - & - & \\
\hline 0006 & F2: & - & - & - & - & - & 5.69 & - & - & - & \\
\hline 0007 & $\mathrm{O} 4 \mathrm{~V}((\mathrm{f}+))$ & 42800 & M06 & 3.95 & 120 & 113 & 0.56 & 5.54 & 38.4 & 2.4 & SB1 \\
\hline $0008^{*}$ & B1e & 27300 & $\mathrm{AM}$ & 3.05 & 299 & 295 & 5.74 & 4.96 & 17.6 & 7.8 & \\
\hline $0009^{*}$ & B0e & 32000 & $\mathrm{AM}$ & 3.25 & 199 & 187 & 3.74 & 5.07 & 19.8 & 7.2 & \\
\hline 0010 & O7 IIIn((f)) & 35900 & M06 & 3.54 & 313 & 339 & 6.88 & 5.21 & 25.8 & 4.2 & \\
\hline 0011 & B9 II & - & - & - & - & - & 9.17 & - & - & - & \\
\hline 0012 & $\mathrm{~B} 1 \mathrm{Ib}$ & 24200 & $\mathrm{H} 08$ & 3.20 & $\leq 40$ & $\leq 40$ & 4.82 & 4.77 & 14.6 & 11.1 & \\
\hline $0013^{*}$ & B1: & 27300 & $\mathrm{AM}$ & 3.75 & 120 & 121 & 2.34 & 4.89 & 17.4 & 8.5 & SB2 \\
\hline 0014 & A0 II & - & - & - & - & - & 4.99 & - & - & - & \\
\hline 0015 & B1 V & 27300 & A & 3.85 & - & - & 8.47 & 4.79 & 15.8 & 9.2 & SB2 \\
\hline 0016 & B0.5 Vn & 29650 & A & 3.95 & 181 & 163 & 6.04 & 4.87 & 17.8 & 7.6 & SB2 \\
\hline $0017^{*}$ & $\mathrm{Be}(\mathrm{B} 1)$ & 27300 & $\mathrm{AM}$ & 3.25 & 231 & 226 & 7.54 & 4.77 & 15.8 & 9.1 & SB1 \\
\hline 0018 & O9.5 IIIe & 32700 & M06 & 3.33 & 138 & 130 & 2.93 & 4.93 & 19.2 & 6.0 & \\
\hline 0019 & A0 II & - & - & - & - & - & 2.37 & - & - & - & \\
\hline 0020 & B1 V+early-B & 27300 & A & 3.75 & - & - & 6.41 & 4.68 & 14.6 & 10.1 & SB2 \\
\hline 0021 & B1 III & 25150 & $\mathrm{H} 08$ & 3.50 & $\leq 40$ & $\leq 40$ & 4.58 & 4.60 & 15.0 & 10.0 & \\
\hline 0022 & O9 V & 36800 & M06 & 4.20 & 55 & 58 & 1.32 & 5.02 & 22.6 & 4.1 & \\
\hline $0023^{*}$ & B0.2: (Be-Fe) & 30800 & $\mathrm{AM}$ & 3.75 & 65 & 57 & 1.94 & 4.81 & 18.8 & 5.8 & \\
\hline $0024^{*}$ & B2: shell(Be-Fe) & 24950 & $\mathrm{AM}$ & 3.10 & 190 & 197 & 2.67 & 5.00 & 17.2 & 8.4 & \\
\hline 0025 & O9 V & 36200 & M06 & 4.07 & 138 & 138 & 3.73 & 4.99 & 21.8 & 4.3 & SB1 \\
\hline 0026 & B0 IV (Nstr) & 31000 & H08 & 3.70 & 75 & 68 & 3.87 & 4.79 & 17.0 & 7.3 & \\
\hline 0027 & B $0.5 \mathrm{~V}$ & 31000 & $\mathrm{H} 08$ & 4.05 & 220 & 241 & 3.12 & 4.78 & 17.2 & 7.1 & \\
\hline 0028 & OC6 Vz & 42900 & M06 & 3.97 & $\leq 40$ & $\leq 40$ & 2.56 & 5.16 & 30.4 & 1.2 & \\
\hline 0029 & B0 V & 32150 & $\mathrm{H} 08$ & 4.10 & $\leq 40$ & $\leq 40$ & 1.76 & 4.82 & 17.6 & 6.7 & SB1 \\
\hline 0030 & B0 V & 32000 & A & 4.15 & 183 & 181 & 4.27 & 4.81 & 17.8 & 6.6 & SB2 \\
\hline 0031 & $\mathrm{O} 8 \mathrm{Vz}$ & 39500 & M06 & 3.99 & $\leq 40$ & $\leq 40$ & 7.01 & 5.06 & 25.2 & 2.9 & \\
\hline 0032 & B $0.5 \mathrm{~V}$ & 29000 & H08 & 4.40 & 125 & 118 & 5.83 & 4.68 & 15.2 & 8.8 & SB1 \\
\hline 0033 & $\mathrm{O} 8 \mathrm{~V}$ & 39900 & M06 & 4.44 & 188 & 170 & 0.71 & 5.05 & 25.8 & 2.4 & \\
\hline 0034 & $08.5 \mathrm{~V}$ & 37400 & I & - & 206 & 190 & 0.44 & 4.97 & 22.8 & 3.6 & SB1 \\
\hline 0035 & $\mathrm{~B} 1 \mathrm{~V}$ & 27300 & A & 3.75 & 145 & - & 5.84 & 4.60 & 14.0 & 10.3 & SB2 \\
\hline 0036 & B0.5 V(Be-Fe) & 29650 & A & 3.45 & 287 & 276 & 2.15 & 4.68 & 15.8 & 8.0 & \\
\hline 0037 & B3 III & 18800 & $\mathrm{H} 08$ & 3.20 & $\leq 40$ & $\leq 40$ & 4.07 & 4.20 & 8.8 & 24.4 & \\
\hline 0038 & B1 V & 27300 & A & 4.00 & 158 & 160 & 5.34 & 4.57 & 12.8 & 13.1 & \\
\hline 0039 & B0.7 V & 25800 & H08 & 3.60 & $\leq 40$ & $\leq 40$ & 8.60 & 4.51 & 12.6 & 12.6 & SB1 \\
\hline 0040 & B $0.2 \mathrm{~V}$ & 30600 & H08 & 4.00 & $\leq 40$ & $\leq 40$ & 3.46 & 4.67 & 15.4 & 8.0 & SB1 \\
\hline 0041 & B2 (Be-Fe) & 24950 & A & 3.85 & 144 & 144 & 8.42 & 4.46 & 12.2 & 13.4 & \\
\hline 0042 & A7 II & - & - & - & - & - & 8.39 & - & - & - & \\
\hline 0043 & B $0 \mathrm{~V}$ & 33000 & $\mathrm{H} 08$ & 4.25 & $\leq 40$ & $\leq 40$ & 4.02 & 4.71 & 17.0 & 6.2 & \\
\hline 0044 & B1 II & 23000 & H08 & 3.50 & $\leq 40$ & $\leq 40$ & 3.25 & 4.33 & 10.6 & 17.2 & \\
\hline 0045 & B0.5 Vne & 29650 & A & 3.75 & 181 & 173 & 3.89 & 4.56 & 14.4 & 8.5 & \\
\hline 0046 & $\mathrm{O} 7 \mathrm{Vn}$ & 39700 & M06 & 4.17 & 340 & 378 & 3.80 & 4.90 & 25.0 & 1.0 & \\
\hline 0047 & B2.5 III & 19850 & H08 & 3.25 & 63 & 55 & 9.35 & 4.15 & 9.0 & 23.7 & \\
\hline 0048 & Be (B3 shell) & 21500 & EM & 3.15 & $\leq 40$ & $\leq 40$ & 2.55 & 4.22 & 9.6 & 20.7 & \\
\hline 0049 & B8 II & 13000 & H08 & 2.70 & 72 & 64 & 7.70 & 3.70 & - & - & \\
\hline 0050 & O8 Vn & 37200 & M06 & 4.16 & 357 & 340 & 1.48 & 4.80 & 21.6 & 2.5 & \\
\hline 0051 & $\mathrm{O} 7 \mathrm{Vz}$ & 41600 & M06 & 4.33 & $\leq 40$ & $\leq 40$ & 0.37 & 4.93 & 26.4 & 0.0 & \\
\hline 0052 & B $1.5 \mathrm{~V}$ & 26100 & A & 3.85 & $\leq 40$ & $\leq 40$ & 6.93 & 4.39 & 11.8 & 13.1 & SB2 \\
\hline
\end{tabular}

Notes. The fourth column indicates the method used to estimate the effective temperature, viz. H08: taken from Hunter et al. (2008a); M06: taken from Mokiem et al. (2006); A: adopted from effective temperature scales of Trundle et al. (2007); I: interpolated from these temperature scales; E: extrapolated from these temperature scales; M: luminosity class V assumed; Si: silicon ionization balance; He: He I line at $4026 \AA$; He+: He II lines at 4541 and $4686 \AA$; L: literature. Two methods were used to estimate the $v_{\mathrm{e}} \sin i$ : profile fitting (PF) and Fourier transform (FT). Targets marked with an asterisk appear to be overluminous for an assumed luminosity class V and their estimates should be treated with caution. SB1 in the final column indicates that significant radial velocity variables were detected while the double-lined spectroscopic binaries are designated as SB2 (and one tripled-lined system as SB3). Many of the Be-type stars from the FSMS were classified by (Evans et al. 2006) as "(Be-Fe)" to indicate Fe II emission. This spectral qualifier has not been used further elsewhere (including for the Survey spectra here) but for completeness cf. the published types, these suffixes are included below. 
Table A.2. continued.

\begin{tabular}{|c|c|c|c|c|c|c|c|c|c|c|c|}
\hline \multirow[t]{2}{*}{ Star } & \multirow[t]{2}{*}{ Spectral Type } & \multirow{2}{*}{$\begin{array}{l}T_{\text {eff }} \\
(\mathrm{K})\end{array}$} & \multirow[t]{2}{*}{ Method } & \multirow{2}{*}{$\begin{array}{l}\log g \\
(\mathrm{dex})\end{array}$} & \multicolumn{2}{|c|}{$v_{\mathrm{e}} \sin i$} & \multirow{2}{*}{$\begin{array}{r}r \\
\left({ }^{\prime}\right) \\
\end{array}$} & \multirow{2}{*}{$\begin{array}{r}\log \mathrm{L} \\
\left(L_{\odot}\right)\end{array}$} & \multirow{2}{*}{$\begin{array}{l}\text { Mass } \\
\left(M_{\odot}\right)\end{array}$} & \multirow{2}{*}{$\begin{array}{r}\text { Age } \\
(\mathrm{Myr})\end{array}$} & \multirow[t]{2}{*}{ Comment } \\
\hline & & & & & $\begin{array}{l}\mathrm{PF} \\
(\mathrm{km}\end{array}$ & $\begin{array}{l}\text { FT } \\
-1)\end{array}$ & & & & & \\
\hline 0053 & B0.5 V & 29500 & H08 & 3.75 & 170 & 174 & 7.60 & 4.51 & 14.0 & 8.7 & SB1 \\
\hline 0054 & B1 V & 29000 & H08 & 4.30 & $\leq 40$ & $\leq 40$ & 2.88 & 4.47 & 13.2 & 9.8 & \\
\hline 0055 & B $0.5 \mathrm{~V}$ & 29500 & H08 & 4.00 & 130 & 137 & 3.20 & 4.49 & 13.8 & 8.9 & \\
\hline 0056 & B0 V & 31000 & H08 & 3.80 & $\leq 40$ & $\leq 40$ & 1.06 & 4.55 & 14.6 & 7.8 & \\
\hline 0057 & B2.5 III & 19850 & $\mathrm{H} 08$ & 3.35 & 73 & 80 & 5.38 & 4.08 & 8.6 & 25.6 & \\
\hline 0058 & B $0.5 \mathrm{~V}$ & 29500 & H08 & 4.25 & 180 & 192 & 3.40 & 4.47 & 13.6 & 8.7 & SB1 \\
\hline 0059 & A5 II & - & - & - & - & - & 5.33 & - & - & - & \\
\hline 0060 & B0.5e (shell) & 29650 & $\mathrm{AM}$ & 3.40 & 311 & 286 & 4.75 & 4.46 & 14.0 & 8.1 & \\
\hline 0061 & B1-2 (Be-Fe) & 26100 & IM & 3.55 & 336 & 329 & 5.68 & 4.32 & 11.8 & 11.9 & \\
\hline 0062 & B $0.2 \mathrm{~V}$ & 29750 & H08 & 4.00 & $\leq 40$ & $\leq 40$ & 6.61 & 4.45 & 13.4 & 9.0 & \\
\hline 0063 & A0 II & - & - & - & - & - & 5.69 & - & - & - & \\
\hline 0064 & B1-2 (Be-Fe) & 26100 & IM & 3.85 & 108 & 120 & 5.44 & 4.31 & 11.4 & 13.3 & \\
\hline 0065 & $\mathrm{~B} 3(\mathrm{Be}-\mathrm{Fe})$ & 21500 & EM & 3.25 & 222 & 238 & 1.88 & 4.12 & 9.4 & 20.9 & \\
\hline 0066 & O9.5 V & 35600 & M06 & 4.25 & 129 & 116 & 1.86 & 4.65 & 18.4 & 3.2 & \\
\hline 0067 & B1-2 (Be-Fe) & 26100 & $\mathrm{IM}$ & 3.35 & 351 & 339 & 6.19 & 4.30 & 11.8 & 11.9 & \\
\hline 0068 & B0 V (Be-Fe) & 32000 & A & 3.85 & 378 & 384 & 5.60 & 4.51 & 15.8 & 5.4 & \\
\hline 0069 & B1-2 (Be-Fe) & 26100 & IM & 3.75 & 186 & 170 & 9.43 & 4.28 & 11.2 & 13.2 & \\
\hline 0070 & B $0.5 \mathrm{~V}$ & 30500 & H08 & 4.15 & 109 & 102 & 3.56 & 4.43 & 13.8 & 13.8 & \\
\hline 0071 & A0 II & - & - & - & - & - & 7.85 & - & - & - & \\
\hline 0072 & B1-2 (Be-Fe) & 26100 & IM & 3.85 & 102 & 95 & 3.32 & 4.26 & 11.0 & 13.6 & \\
\hline 0073 & B1-2 (Be-Fe) & 26100 & IM & 3.80 & 190 & 179 & 7.21 & 4.26 & 11.2 & 13.2 & \\
\hline 0074 & B3 III & 16300 & $\mathrm{H} 08$ & 3.20 & 52 & 62 & 8.30 & 3.79 & 6.6 & 40.8 & \\
\hline 0075 & B1 V & 27700 & H08 & 4.30 & $\leq 40$ & $\leq 40$ & 4.68 & 4.31 & 11.8 & 11.6 & SB1 \\
\hline 0076 & $\mathrm{~B} 2(\mathrm{Be}-\mathrm{Fe})$ & 24950 & $\mathrm{AM}$ & 3.40 & 237 & 247 & 1.88 & 4.21 & 10.6 & 14.6 & \\
\hline 0077 & O9 V & 36500 & M06 & 3.99 & 177 & 169 & 1.31 & 4.62 & 19.0 & 1.2 & \\
\hline 0078 & B2 III & 21200 & A & 3.60 & 154 & 163 & 5.43 & 4.05 & 8.8 & 23.0 & \\
\hline 0079 & B0.5 Vn & 29500 & H08 & 4.20 & 293 & 308 & 0.34 & 4.37 & 13.4 & 8.0 & \\
\hline 0080 & B1 V & 27300 & H08 & 4.25 & 216 & 203 & 1.14 & 4.28 & 11.6 & 11.4 & \\
\hline 0081 & B2 IIIn & 21200 & $\mathrm{H} 08$ & 3.50 & 255 & 260 & 5.61 & 4.03 & 8.8 & 22.4 & \\
\hline 0082 & B2 III & 21200 & H08 & 3.70 & 168 & 166 & 5.78 & 4.03 & 8.8 & 23.0 & SB1 \\
\hline 0083 & B1 V & 27300 & H08 & 4.05 & 207 & 200 & 1.83 & 4.28 & 11.6 & 11.4 & \\
\hline 0084 & B1 V & 27300 & H08 & 4.25 & 105 & 95 & 5.88 & 4.27 & 11.4 & 11.9 & \\
\hline 0085 & B2 III & 21200 & A & 3.55 & $\leq 40$ & $\leq 40$ & 6.63 & 4.03 & 8.6 & 24.6 & SB2 \\
\hline 0086 & B0.2 V & 30800 & A & 4.25 & 188 & 190 & 5.09 & 4.54 & 17.2 & 2.4 & \\
\hline 0091 & B1e & 27300 & $\mathrm{AM}$ & 3.85 & 49 & 52 & 8.68 & 4.25 & 11.4 & 12.1 & SB1 \\
\hline 0092 & B1 Vn & 27300 & H08 & 3.95 & 234 & 240 & 4.14 & 4.24 & 11.6 & 11.1 & \\
\hline 0093 & B0 V & 34400 & M06 & 4.40 & 187 & 196 & 0.74 & 4.50 & 16.6 & 2.6 & \\
\hline 0094 & B $0.7 \mathrm{~V}$ & 28500 & H08 & 4.00 & $\leq 40$ & $\leq 40$ & 5.28 & 4.28 & 11.8 & 10.5 & \\
\hline 0095 & B1-2 (Be-Fe) & 26100 & IM & 3.90 & 227 & 236 & 8.43 & 4.18 & 10.8 & 13.1 & \\
\hline 0096 & B1-2 (Be-Fe) & 26100 & IM & 3.35 & 343 & 337 & 8.38 & 4.18 & 11.0 & 12.1 & \\
\hline 0097 & $\mathrm{O} 9 \mathrm{~V}$ & 37500 & M06 & 4.49 & $\leq 40$ & $\leq 40$ & 0.86 & 4.58 & 19.2 & 0.0 & \\
\hline 0098 & B $1.5 \mathrm{~V}$ & 26100 & $\mathrm{H} 08$ & 4.05 & 56 & 60 & 2.70 & 4.16 & 10.4 & 14.2 & \\
\hline 0099 & B3 III & 18000 & H08 & 3.40 & 94 & 98 & 8.63 & 3.79 & 7.0 & 36.8 & \\
\hline 0100 & B $1.5 \mathrm{~V}$ & 26100 & H08 & 4.30 & 183 & 174 & 6.89 & 4.16 & 10.6 & 13.6 & \\
\hline 0101 & B1 V & 27300 & H08 & 4.25 & $\leq 40$ & $\leq 40$ & 4.96 & 4.18 & 11.0 & 12.4 & \\
\hline 0102 & B3 III & 17700 & $\mathrm{H} 08$ & 3.70 & $\leq 40$ & $\leq 40$ & 5.34 & 3.75 & 6.8 & 40.9 & \\
\hline 0103 & B0.5 V & 29500 & H08 & 4.00 & $\leq 40$ & $\leq 40$ & 7.54 & 4.26 & 12.2 & 8.8 & \\
\hline 0104 & B0 V & 32500 & $\mathrm{H} 08$ & 4.35 & $\leq 40$ & $\leq 40$ & 1.19 & 4.37 & 14.4 & 4.5 & SB1 \\
\hline 0105 & B2 III & 21200 & A & 3.50 & 193 & 208 & 3.50 & 3.92 & 8.2 & 24.8 & SB1 \\
\hline 0106 & B1 V & 27500 & $\mathrm{H} 08$ & 4.20 & 142 & 135 & 7.49 & 4.17 & 11.0 & 11.4 & SB1 \\
\hline 0107 & $\mathrm{O} 9.5 \mathrm{~V}$ & 35900 & M06 & 4.23 & 55 & 60 & 0.45 & 4.48 & 17.4 & 0.0 & \\
\hline 0108 & B1.5 V & 26100 & H08 & 3.95 & 167 & 171 & 4.59 & 4.11 & 10.4 & 13.7 & \\
\hline 0109 & B $1.5 \mathrm{~V}$ & 26100 & $\mathrm{H} 08$ & 4.25 & 123 & 119 & 6.03 & 4.11 & 10.4 & 13.7 & \\
\hline 0110 & B1-2 (Be-Fe) & 26100 & I & 3.95 & 243 & 232 & 5.91 & 4.11 & 10.4 & 13.7 & SB1 \\
\hline 0111 & B $0.5 \mathrm{~V}$ & 28000 & $\mathrm{He}+$ & 4.10 & 57 & 49 & 0.49 & 4.18 & 10.8 & 10.7 & \\
\hline 0112 & $\mathrm{O} 9.5 \mathrm{~V}$ & 34400 & M06 & 4.15 & 143 & 141 & 1.76 & 4.41 & 16.0 & 0.8 & \\
\hline 0113 & B $0.5 \mathrm{~V}$ & 29650 & A & 4.00 & 122 & 132 & 4.10 & 4.22 & 12.2 & 7.6 & \\
\hline 0114 & B1 Vn & 27300 & H08 & 3.90 & 287 & 291 & 5.16 & 4.13 & 11.2 & 10.4 & \\
\hline 0115 & B $0.2 \mathrm{~V}$ & 30800 & A & 4.05 & 133 & 120 & 0.11 & 4.25 & 13.0 & 5.7 & \\
\hline 0116 & B1 V & 28250 & $\mathrm{H} 08$ & 4.10 & $\leq 40$ & $\leq 40$ & 6.26 & 4.15 & 11.2 & 10.4 & \\
\hline 1001 & O4-6 If +? & - & - & - & 180 & 156 & 0.00 & - & - & - & SB2 \\
\hline 1002 & B1.5 Ia & 20650 & A & 2.55 & 67 & 51 & 6.39 & 5.25 & - & - & SB1 \\
\hline
\end{tabular}


Table A.2. continued.

\begin{tabular}{|c|c|c|c|c|c|c|c|c|c|c|c|}
\hline \multirow[t]{2}{*}{ Star } & \multirow[t]{2}{*}{ Spectral Type } & \multirow{2}{*}{$\begin{array}{l}T_{\text {eff }} \\
(\mathrm{K}) \\
\end{array}$} & \multirow[t]{2}{*}{ Method } & \multirow{2}{*}{$\begin{array}{l}\log g \\
(\mathrm{dex})\end{array}$} & \multicolumn{2}{|c|}{$v_{\mathrm{e}} \sin i$} & \multirow[b]{2}{*}{$\left({ }^{\prime}\right)$} & \multirow{2}{*}{$\begin{array}{r}\log \mathrm{L} \\
\left(L_{\odot}\right)\end{array}$} & \multirow{2}{*}{$\begin{array}{l}\text { Mass } \\
\left(M_{\odot}\right)\end{array}$} & \multirow{2}{*}{$\begin{array}{r}\text { Age } \\
\text { (Myr) }\end{array}$} & \multirow[t]{2}{*}{ Comment } \\
\hline & & & & & $\begin{array}{l}\mathrm{PF} \\
(\mathrm{kn}\end{array}$ & $\begin{array}{l}\text { FT } \\
-1)\end{array}$ & & & & & \\
\hline 1003 & B3 Iab & 15700 & $\mathrm{Si}$ & 2.15 & 43 & $\leq 40$ & 6.79 & 4.91 & _- & - & \\
\hline 1004 & A2 Ib & - & - & - & - & - & 9.13 & - & - & - & \\
\hline 1005 & $\mathrm{~A} 2 \mathrm{Ib}$ & - & - & - & - & - & 9.33 & - & - & - & \\
\hline 1006 & A2 Ib & - & - & - & - & - & 4.7 & - & - & - & \\
\hline 1007 & B1 Ia & 21500 & $\mathrm{Si}$ & 2.65 & 79 & 66 & 2.97 & 5.03 & 17.6 & 9.1 & SB 1 \\
\hline 1008 & $\mathrm{O} 2 \mathrm{III}\left(\mathrm{f}^{a} s t\right)$ & 51700 & $\mathrm{~L}$ & 4.00 & 110 & 130 & 0.29 & 6.00 & 57.4 & 1.9 & \\
\hline $1009^{*}$ & B1e & 27300 & AM & 3.25 & 75 & 81 & 4.22 & 5.20 & 22.8 & 6.6 & \\
\hline 1010 & O6: V-III((f)) & 42970 & A & - & - & - & 0.40 & 5.70 & 45.0 & 2.3 & SB3 \\
\hline 1011 & O9 III & 33800 & $\mathrm{Si}$ & 3.75 & 77 & 86 & 5.06 & 5.38 & 28.2 & 4.5 & SB1 \\
\hline 1012 & O6: V((f)) & 39300 & $\mathrm{~L}$ & 3.75 & 60 & 58 & 0.23 & 5.40 & 32.2 & 3.1 & SB1 \\
\hline $1013^{*}$ & $0.5 \mathrm{e}$ & 29650 & $\mathrm{AM}$ & 3.65 & 245 & 259 & 0.50 & 5.02 & 19.8 & 6.8 & \\
\hline 1014 & B3 Ib + mid A? & 15500 & A & 2.25 & $\leq 40$ & $\leq 40$ & 4.76 & 4.32 & - & - & SB2? \\
\hline 1015 & $\mathrm{O} 7 \mathrm{~V}$ & 40730 & A & - & 119 & 135 & 0.21 & 5.33 & 30.8 & 2.9 & \\
\hline 1016 & B8 Ib & 12500 & $\mathrm{He}$ & 2.45 & 44 & $\leq 40$ & 2.45 & 4.08 & - & - & \\
\hline 1017 & $\mathrm{O} 9 \mathrm{~V}$ & 36265 & A & - & 260 & 239 & 5.95 & 5.19 & 25.6 & 4.2 & SB2 \\
\hline $1018^{*}$ & B0ne (shell) & 32000 & $\mathrm{AM}$ & 3.50 & 290 & 313 & 9.73 & 5.03 & 21.0 & 5.9 & \\
\hline 1019 & O6.5f?p & 37500 & $\mathrm{~L}$ & 3.75 & $\leq 40$ & 46 & 4.63 & 5.22 & 26.6 & 3.9 & \\
\hline 1020 & B1 III & 23950 & A & 3.50 & 90 & 81 & 6.19 & 4.71 & 14.0 & 11.8 & SB 1 \\
\hline 1021 & B2 II & 20075 & AI & 3.25 & 152 & 143 & 3.60 & 4.49 & 11.2 & 17.2 & \\
\hline 1022 & B2 II & 20075 & $\mathrm{AI}$ & 3.00 & 190 & 187 & 8.87 & 4.49 & 11.2 & 17.2 & \\
\hline 1023 & B0 V & 31750 & $\mathrm{He}+$ & 3.90 & 175 & 178 & 3.40 & 4.95 & 19.4 & 6.3 & SB1 \\
\hline 1024 & B & - & - & - & - & - & 1.99 & - & - & - & \\
\hline 1025 & O7 V & 40730 & A & - & 218 & 257 & 0.97 & 5.24 & 29.6 & 2.7 & \\
\hline 1026 & O6.5 V((f)) & 41850 & A & - & $\leq 40$ & 46 & 0.94 & 5.26 & 30.8 & 2.4 & \\
\hline $1027^{*}$ & B0-3e & 26100 & $\mathrm{AM}$ & 3.75 & 246 & 258 & 9.26 & 4.71 & 15.0 & 9.9 & \\
\hline 1028 & B2.5 III & 22000 & $\mathrm{Si}$ & 3.45 & $\leq 40$ & $\leq 40$ & 7.75 & 4.29 & 10.2 & 18.8 & \\
\hline 1029 & B2.5 III & 19850 & A & 3.25 & 53 & 55 & 6.48 & 4.38 & 10.0 & 19.9 & \\
\hline 1030 & OC5 V & 45200 & A & - & $\leq 40$ & - & 1.15 & 5.27 & 35.6 & 0.4 & \\
\hline $1031^{*}$ & B2-3e & 24290 & EM & 3.30 & 215 & 233 & 2.88 & 4.55 & 13.0 & 12.4 & \\
\hline $1032^{*}$ & B0.5:e & 29650 & $\mathrm{AM}$ & 3.55 & 367 & 345 & 8.51 & 4.73 & 16.0 & 7.6 & \\
\hline 1033 & B0 V & 33500 & $\mathrm{He}+$ & 4.40 & 312 & 310 & 0.36 & 4.87 & 19.4 & 5.4 & \\
\hline 1034 & O9.5-B0 V & 35000 & I & 4.35 & 378 & 375 & 0.79 & 4.92 & 21.0 & 4.6 & \\
\hline 1035 & $07.5 \mathrm{~V}((\mathrm{f}))$ & 39615 & I & - & 82 & 98 & 0.44 & 5.06 & 25.4 & 2.7 & \\
\hline 1036 & B2.5 III-II & 19850 & A & 3.25 & 50 & 57 & 4.39 & 4.31 & 9.8 & 20.7 & \\
\hline 1037 & B0 III & 27700 & $\mathrm{Si}$ & 3.75 & 83 & 81 & 6.51 & 4.63 & 14.2 & 10.0 & \\
\hline 1038 & B0: e & 32000 & $\mathrm{AM}$ & - & 380 & 372 & 6.90 & 4.80 & 18.2 & 6.2 & \\
\hline 1039 & $09.5 \mathrm{~V}$ & 35150 & A & - & 149 & 158 & 0.37 & 4.91 & 20.4 & 4.8 & SB1 \\
\hline 1040 & Early-B+Early-B & - & - & - & - & - & 8.69 & - & - & - & SB2 \\
\hline 1041 & B2 III-II & 22500 & $\mathrm{Si}$ & 3.35 & $\leq 40$ & $\leq 40$ & 8.29 & 4.43 & 11.2 & 16.7 & \\
\hline 1042 & B1-2e & 26100 & IM & 3.55 & 266 & 260 & 1.86 & 4.55 & 13.4 & 11.1 & \\
\hline 1043 & B0 V & 32000 & $\mathrm{He}+$ & 4.30 & 147 & 135 & 2.82 & 4.76 & 17.0 & 6.7 & \\
\hline 1044 & B1 III & 23950 & A & 3.75 & 132 & 115 & 8.33 & 4.46 & 12.0 & 14.3 & \\
\hline 1045 & O9 V & 36265 & A & - & 83 & 87 & 0.22 & 4.90 & 21.0 & 4.2 & SB1 \\
\hline 1046 & B $0.5 \mathrm{~V}$ & 29650 & A & 3.90 & 281 & 270 & 6.92 & 4.65 & 15.4 & 8.0 & \\
\hline 1047 & A5 III & - & - & - & - & - & 7.04 & - & - & - & \\
\hline 1048 & O9 V & 36265 & A & - & 124 & 136 & 0.62 & 4.88 & 20.8 & 4.1 & \\
\hline 1049 & B0 V & 31000 & $\mathrm{He}+$ & 4.25 & 293 & 282 & 0.49 & 4.74 & 16.8 & 7.0 & SB2 \\
\hline 1050 & B1.5 II & 24100 & $\mathrm{Si}$ & 3.45 & $\leq 40$ & $\leq 40$ & 7.02 & 4.44 & 11.6 & 14.6 & \\
\hline 1051 & O9 V & 36265 & A & - & $\leq 40$ & $\leq 40$ & 5.78 & 4.88 & 20.6 & 4.2 & \\
\hline 1052 & B0 V & 34000 & $\mathrm{He}+$ & 4.40 & 108 & 120 & 0.30 & 4.78 & 18.2 & 5.4 & \\
\hline 1053 & B0.5 III & 27500 & $\mathrm{Si}$ & 3.60 & $\leq 40$ & $\leq 40$ & 6.44 & 4.50 & 12.8 & 11.8 & \\
\hline 1054 & B1 III & 23950 & A & 3.45 & 222 & 234 & 4.85 & 4.40 & 11.6 & 14.4 & SB1 \\
\hline 1055 & $09.5 \mathrm{~V}$ & 35150 & A & - & 53 & 72 & 0.70 & 4.80 & 19.2 & 4.7 & \\
\hline 1056 & A0 II & - & - & - & - & - & 7.43 & - & - & - & \\
\hline 1057 & B0 V & 33000 & $\mathrm{He}+$ & 4.10 & 105 & 128 & 4.18 & 4.72 & 17.2 & 6.0 & \\
\hline 1058 & O8 Vn & 38500 & A & - & 290 & 316 & 0.46 & 4.90 & 23.6 & 2.2 & \\
\hline 1059 & B0 V & 30000 & $\mathrm{He}+$ & 4.05 & 58 & 62 & 3.17 & 4.62 & 16.2 & 5.9 & SB1 \\
\hline 1060 & $\mathrm{O} 8 \mathrm{~V}$ & 38500 & A & - & 141 & 148 & 0.64 & 4.88 & 22.8 & 2.3 & SB1 \\
\hline 1061 & $\mathrm{~B} 0 \mathrm{~V}$ & 31750 & $\mathrm{He}+$ & 4.05 & 139 & 132 & 9.06 & 4.65 & 16.0 & 6.9 & \\
\hline 1062 & B1.5 V (shell) & 26100 & A & 3.95 & 114 & 129 & 4.87 & 4.42 & 12.2 & 12.4 & \\
\hline 1063 & B5e & - & - & - & 165 & 164 & 6.49 & - & - & - & \\
\hline 1064 & B1.5 III & 22550 & A & 3.60 & 146 & 137 & 8.86 & 4.26 & 10.2 & 18.3 & SB1 \\
\hline
\end{tabular}


Table A.2. continued.

\begin{tabular}{|c|c|c|c|c|c|c|c|c|c|c|c|}
\hline \multirow[t]{2}{*}{ Star } & \multirow[t]{2}{*}{ Spectral Type } & \multirow{2}{*}{$\begin{array}{l}T_{\text {eff }} \\
(\mathrm{K}) \\
\end{array}$} & \multirow[t]{2}{*}{ Method } & \multirow{2}{*}{$\begin{array}{l}\log g \\
(\mathrm{dex}) \\
\end{array}$} & \multicolumn{2}{|c|}{$v_{\mathrm{e}} \sin i$} & \multirow[b]{2}{*}{$\left({ }^{\prime}\right)$} & \multirow{2}{*}{$\begin{array}{r}\log \mathrm{L} \\
\left(L_{\odot}\right)\end{array}$} & \multirow{2}{*}{$\begin{array}{l}\text { Mass } \\
\left(M_{\odot}\right)\end{array}$} & \multirow{2}{*}{$\begin{array}{r}\text { Age } \\
\text { (Myr) }\end{array}$} & \multirow[t]{2}{*}{ Comment } \\
\hline & & & & & $\begin{array}{l}\mathrm{PF} \\
(\mathrm{km}\end{array}$ & $\begin{array}{l}\text { FT } \\
-1)\end{array}$ & & & & & \\
\hline 1065 & A0 II & - & - & - & - & - & 1.96 & - & - & - & \\
\hline 1066 & B1-2e & 26100 & IM & 3.65 & 226 & 222 & 4.92 & 4.39 & 12.2 & 12.1 & \\
\hline 1067 & O9 V & 36265 & A & - & 56 & 66 & 8.10 & 4.76 & 19.6 & 3.5 & \\
\hline 1068 & B0.7 III & 25500 & $\mathrm{He}+$ & 3.85 & 52 & 60 & 5.97 & 4.35 & 11.4 & 14.2 & \\
\hline 1069 & B0: + B0.5: & - & - & - & - & - & 8.08 & - & - & - & SB2 \\
\hline 1070 & B2 III & 21200 & A & 3.40 & $\leq 40$ & $\leq 40$ & 1.31 & 4.18 & 9.4 & 22.1 & \\
\hline 1071 & $06.5 \mathrm{Vz}$ & 38200 & A & - & $\leq 40$ & $\leq 40$ & 0.79 & 4.92 & 26.2 & 0.0 & \\
\hline 1072 & B $0.2 \mathrm{~V}$ & 31750 & $\mathrm{He}+$ & 4.35 & 163 & 166 & 7.82 & 4.59 & 15.6 & 6.8 & \\
\hline 1073 & B0 V & 31750 & $\mathrm{He}+$ & 4.40 & 297 & 318 & 0.22 & 4.59 & 16.0 & 6.2 & \\
\hline 1074 & B0.5 V & 29000 & $\mathrm{He}+$ & 4.35 & 281 & 253 & 0.65 & 4.47 & 13.6 & 8.9 & \\
\hline 1075 & B1 III & 23950 & A & 3.50 & 104 & 97 & 5.18 & 4.28 & 10.6 & 16.3 & \\
\hline 1076 & B1 III & 26500 & $\mathrm{Si}$ & 3.90 & 65 & 69 & 7.26 & 4.36 & 11.8 & 12.7 & \\
\hline 1077 & B2.5 III & 19850 & A & 3.55 & 221 & 230 & 6.23 & 4.08 & 8.8 & 24.0 & \\
\hline 1078 & B0.5 III & 27500 & $\mathrm{He}+$ & 3.80 & 105 & 104 & 5.73 & 4.40 & 12.4 & 11.2 & \\
\hline 1079 & B0 III & 31000 & $\mathrm{He}+$ & 4.00 & $\leq 40$ & $\leq 40$ & 0.77 & 4.50 & 14.2 & 7.7 & \\
\hline 1080 & B0.5 III & 27800 & $\mathrm{Si}$ & 3.90 & $\leq 40$ & $\leq 40$ & 6.15 & 4.40 & 12.4 & 11.3 & \\
\hline 1081 & B1 III & 26400 & $\mathrm{Si}$ & 3.75 & $\leq 40$ & $\leq 40$ & 7.67 & 4.35 & 11.5 & 13.1 & \\
\hline 1082 & B0 III & 29000 & $\mathrm{Si}$ & 3.85 & $\leq 40$ & $\leq 40$ & 6.92 & 4.44 & 13.0 & 9.8 & \\
\hline 1083 & B3 III & 16500 & $\mathrm{Si}$ & 3.15 & $\leq 40$ & $\leq 40$ & 3.52 & 3.88 & 7.0 & 38.5 & \\
\hline 1084 & B1.5 III & 22550 & A & 3.70 & 162 & 164 & 6.16 & 4.20 & 10.2 & 18.9 & \\
\hline 1085 & B3 III & 18450 & A & 3.20 & 205 & 183 & 7.96 & 3.98 & 8.0 & 29.1 & \\
\hline 1086 & $\mathrm{O} 8 \mathrm{~V}$ & 38500 & A & - & 163 & 171 & 0.59 & 4.77 & 22.0 & 0.8 & \\
\hline 1087 & B2 III & 21200 & A & 3.50 & 141 & 131 & 2.95 & 4.12 & 9.2 & 22.2 & \\
\hline 1088 & B2.5 III & 19850 & A & 3.30 & 187 & 184 & 5.53 & 4.06 & 8.6 & 24.7 & \\
\hline 1089 & B3e & 21500 & EM & 3.65 & 237 & 254 & 8.82 & 4.12 & 9.4 & 20.3 & \\
\hline 1090 & B0-3 V & 26100 & $\mathrm{IM}$ & 3.95 & 319 & 332 & 0.19 & 4.31 & 11.8 & 12.0 & \\
\hline 1091 & B0.5: + B0.5: & - & - & - & - & - & 6.25 & - & - & - & SB2 \\
\hline 1092 & $\mathrm{~B} 1 \mathrm{~V}$ & 27000 & $\mathrm{He}+$ & 4.10 & 175 & 170 & 3.26 & 4.33 & 11.8 & 11.8 & \\
\hline 1093 & B1 V & 26000 & $\mathrm{He}+$ & 4.00 & $\leq 40$ & $\leq 40$ & 5.75 & 4.28 & 11.0 & 14.1 & \\
\hline 1094 & $\mathrm{O} 8 \mathrm{~V}$ & 38500 & A & - & 124 & 121 & 1.16 & 4.74 & 21.6 & 0.4 & \\
\hline 1095 & $\mathrm{~B} 3 \mathrm{e}$ & 21500 & EM & 3.75 & 241 & 244 & 9.10 & 4.12 & 9.4 & 20.3 & \\
\hline 1096 & B $0.5 \mathrm{~V}$ & 30000 & $\mathrm{He}+$ & 4.40 & 250 & 281 & 1.87 & 4.43 & 13.8 & 7.7 & \\
\hline 1097 & B0.5: + early B & 26100 & A & 3.85 & 254 & 272 & 6.97 & 4.28 & 11.4 & 12.5 & \\
\hline 1099 & A7 III & - & - & - & - & - & 9.43 & - & - & - & \\
\hline 1100 & B1: (Herbig?) & 27300 & AM & 3.90 & 173 & 195 & 0.66 & 4.32 & 12.0 & 11.3 & \\
\hline 1101 & B0.5: & 29650 & $\mathrm{AM}$ & 4.25 & 301 & 311 & 1.42 & 4.40 & 13.6 & 7.9 & SB1 \\
\hline 1102 & B2 (shell) & 24950 & AM & 3.50 & 258 & 264 & 7.09 & 4.22 & 10.8 & 14.4 & \\
\hline 1103 & F8 I & - & - & - & - & - & 2.67 & - & - & - & \\
\hline 1104 & B5 II & 14500 & $\mathrm{He}$ & 2.85 & 63 & 68 & 5.07 & 3.65 & 6.0 & 52.4 & \\
\hline 1105 & B1-3 V & 24950 & I & 3.65 & 281 & 286 & 2.04 & 4.19 & 10.6 & 14.4 & \\
\hline 1106 & A5 III & - & - & - & - & - & 7.08 & - & - & - & \\
\hline 1107 & $09.5 \mathrm{~V}$ & 34500 & $\mathrm{He}+$ & 4.10 & 71 & 79 & 0.53 & 4.55 & 16.8 & 3.6 & \\
\hline 1108 & B2 III & 21200 & A & 3.80 & 99 & 109 & 8.98 & 4.03 & 8.6 & 23.8 & \\
\hline 1109 & B1.5 III & 24000 & $\mathrm{Si}$ & 3.60 & $\leq 40$ & $\leq 40$ & 7.69 & 4.14 & 9.8 & 18.0 & \\
\hline 1110 & B1-3e & 24950 & IM & 3.75 & 255 & 240 & 4.42 & 4.18 & 10.6 & 14.8 & \\
\hline 1111 & A5 III & - & - & - & - & - & 3.39 & - & - & - & \\
\hline 1112 & B0 V & 32100 & $\mathrm{Si}$ & 4.20 & 69 & 76 & 9.61 & 4.43 & 14.6 & 5.8 & \\
\hline 1113 & B3 III & 18450 & A & 3.30 & 50 & 50 & 6.99 & 3.87 & 7.4 & 33.1 & \\
\hline 1114 & O9 V & 36265 & A & - & 250 & 232 & 6.19 & 4.58 & 18.8 & 0.4 & \\
\hline 1115 & Early-Be & - & - & - & 269 & 252 & 6.06 & - & - & - & SB2 \\
\hline 1116 & Early-B+Early-B & - & - & - & - & - & 9.26 & - & - & - & SB2 \\
\hline 1117 & B2 III & 21200 & A & 3.70 & 106 & 130 & 5.25 & 4.00 & 8.6 & 23.8 & \\
\hline 1118 & B2.5e & 23900 & EM & 3.60 & 210 & 194 & 7.43 & 4.10 & 9.8 & 17. & \\
\hline 1119 & B1 V & 23000 & $\mathrm{Si}$ & 3.60 & $\leq 40$ & $\leq 40$ & 4.36 & 4.05 & 9.0 & 208 & \\
\hline 1120 & B8 II & 12500 & $\mathrm{He}$ & 3.05 & 54 & 70 & 9.72 & 3.45 & - & - & \\
\hline 1121 & A0 II & - & - & - & - & - & 6.75 & - & - & - & \\
\hline 1122 & B2.5 III & 19850 & A & 3.60 & 125 & 163 & 5.52 & 3.92 & 8.0 & 27.9 & SB1 \\
\hline 1123 & B3e (shell) & 21500 & $\mathrm{E}$ & 3.35 & 275 & 284 & 7.92 & 4.00 & 8.8 & 21.8 & \\
\hline 1124 & B3 III & 18450 & A & 3.70 & 111 & 126 & 3.73 & 3.84 & 7.4 & 33.0 & \\
\hline 1125 & B0 V & 33000 & $\mathrm{He}+$ & 4.40 & $\leq 40$ & $\leq 40$ & 4.90 & 4.44 & 15.0 & 4.6 & \\
\hline 1126 & B1 V & 27300 & A & 4.20 & 260 & 258 & 2.36 & 4.21 & 11.4 & 10.9 & \\
\hline 1127 & B2-3e & 23900 & EM & 3.55 & 223 & 224 & 2.36 & 4.07 & 9.6 & 17.3 & \\
\hline
\end{tabular}


P. L. Dufton et al.: Stellar parameters and rotational velocities in NGC 346

Table A.2. continued.

\begin{tabular}{|c|c|c|c|c|c|c|c|c|c|c|c|}
\hline \multirow[t]{2}{*}{ Star } & \multirow[t]{2}{*}{ Spectral Type } & \multirow{2}{*}{$\begin{array}{l}T_{\text {eff }} \\
(\mathrm{K}) \\
\end{array}$} & \multirow[t]{2}{*}{ Method } & \multirow{2}{*}{$\begin{array}{l}\log g \\
(\mathrm{dex}) \\
\end{array}$} & \multicolumn{2}{|c|}{$v_{\mathrm{e}} \sin i$} & \multirow{2}{*}{$\begin{array}{r}r \\
\left({ }^{\prime}\right) \\
\end{array}$} & \multirow{2}{*}{$\begin{array}{r}\log \mathrm{L} \\
\left(L_{\odot}\right)\end{array}$} & \multirow{2}{*}{$\begin{array}{l}\text { Mass } \\
\left(M_{\odot}\right)\end{array}$} & \multirow{2}{*}{$\begin{array}{r}\text { Age } \\
\text { (Myr) }\end{array}$} & \multirow[t]{2}{*}{ Comment } \\
\hline & & & & & & $\begin{array}{l}\text { FT } \\
-1 \text { ) }\end{array}$ & & & & & \\
\hline 1128 & O7.5 V((f)) & 39615 & I & - & 56 & 68 & 0.84 & 4.63 & 21.6 & 0.0 & \\
\hline 1129 & $\mathrm{~B} 1.5 \mathrm{~V}$ & 26100 & $\mathrm{~A}$ & 3.95 & 176 & 168 & 2.36 & 4.15 & 10.6 & 13.6 & \\
\hline 1130 & B1-3e & 24950 & IM & 4.00 & 310 & 309 & 2.36 & 4.10 & 10.2 & 14.5 & SB1 \\
\hline 1131 & B3 III & 18450 & A & 3.55 & $\leq 40$ & $\leq 40$ & 6.17 & 3.80 & 7.0 & 36.7 & \\
\hline 1132 & B1 V & 27300 & A & 4.15 & 205 & 225 & 6.41 & 4.18 & 11.2 & 11.2 & \\
\hline 1133 & B1 V & 27300 & A & 4.00 & $\leq 40$ & $\leq 40$ & 7.44 & 4.18 & 11.0 & 12.4 & \\
\hline 1134 & B0:e & 32000 & $\mathrm{AM}$ & 3.95 & 519 & 505 & 2.48 & 4.36 & 15.4 & 4.2 & \\
\hline 1135 & B1 Vn & 27300 & A & 4.40 & 301 & 319 & 4.70 & 4.17 & 11.4 & 10.3 & \\
\hline 1136 & B1-3e & 24950 & IM & 3.85 & 205 & 225 & 6.40 & 4.09 & 10.0 & 15.4 & \\
\hline 1137 & B2 V & 24950 & A & 4.00 & 108 & 99 & 7.14 & 4.09 & 9.8 & 16.3 & \\
\hline 1138 & B1-3e & 24950 & IM & 3.60 & 228 & 208 & 9.37 & 4.08 & 10.0 & 15.5 & \\
\hline 1139 & A2 II & - & - & - & - & - & 2.65 & - & - & - & \\
\hline 1140 & B0 V & 33250 & $\mathrm{He}+$ & 4.15 & 159 & 164 & 1.09 & 4.39 & 15.2 & 2.8 & \\
\hline 1141 & B2 III & 21200 & A & 3.75 & $\leq 40$ & $\leq 40$ & 8.03 & 3.91 & 8.0 & 26.7 & \\
\hline 1142 & B1.5 III & 22550 & A & 3.70 & $\leq 40$ & $\leq 40$ & 2.58 & 3.97 & 8.6 & 22.5 & \\
\hline 1143 & B1.5 V & 26100 & A & 4.50 & 289 & 281 & 4.98 & 4.11 & 10.6 & 12.7 & \\
\hline 1144 & $09.5 \mathrm{~V}$ & 35150 & A & - & $\leq 40$ & 43 & 4.04 & 4.44 & 16.6 & 0.3 & \\
\hline 1145 & B $2.5 \mathrm{~V}$ & 23900 & $\mathrm{E}$ & 4.15 & 102 & 110 & 5.63 & 4.01 & 9.2 & 18.7 & \\
\hline 1146 & B1-3e & 24950 & IM & 3.95 & 261 & 271 & 6.57 & 4.06 & 10.0 & 15.1 & \\
\hline 1147 & B1-3e & 24950 & IM & 4.05 & 259 & 238 & 7.27 & 4.06 & 10.0 & 15.0 & \\
\hline 1148 & B1-3e & 24950 & IM & 4.25 & 221 & 240 & 6.62 & 4.05 & 9.8 & 15.3 & \\
\hline 1149 & B2.5 V & 23900 & $\mathrm{E}$ & 3.90 & 145 & 142 & 6.92 & 4.01 & 9.2 & 18.5 & \\
\hline 1150 & B1 V & 26500 & A & 4.10 & 76 & 77 & 6.37 & 4.10 & 10.4 & 13.4 & \\
\hline 1151 & B1-2e & 26100 & IM & 3.65 & 295 & 301 & 3.91 & 4.09 & 10.6 & 12.4 & \\
\hline 1152 & B9 II & - & - & - & - & - & 9.02 & - & - & - & \\
\hline 1153 & B2 V & 24950 & A & 4.30 & 62 & 60 & 6.55 & 4.04 & 9.6 & 16.7 & \\
\hline 1154 & B1.5 Vn & 26100 & A & 4.10 & 226 & 239 & 6.16 & 4.08 & 10.4 & 13.4 & \\
\hline 1155 & B1.5 III & 22550 & $\mathrm{~A}$ & 3.60 & 111 & 120 & 1.55 & 3.94 & 8.6 & 22.2 & \\
\hline 1156 & B1-3e & 24950 & IM & 3.75 & 313 & 308 & 6.35 & 4.04 & 10.0 & 14.6 & \\
\hline 1157 & A0 III-II & - & - & - & - & - & 4.75 & - & - & - & \\
\hline 1158 & B3 Ve & 21500 & $\mathrm{E}$ & 3.75 & 226 & 239 & 2.84 & 3.88 & 8.2 & 24.0 & \\
\hline 1159 & B $1.5 \mathrm{~V}$ & 26100 & A & 4.30 & 200 & 198 & 0.64 & 4.06 & 10.2 & 13.4 & \\
\hline 1160 & B1 V & 27300 & A & 3.95 & 315 & 340 & 3.11 & 4.10 & 11.2 & 9.7 & \\
\hline 1161 & B2 V & 24950 & A & 4.40 & 108 & 111 & 1.26 & 4.02 & 9.4 & 16.5 & \\
\hline 1162 & A0 II & - & - & - & - & - & 3.31 & - & - & - & \\
\hline 1163 & B1 V & 27300 & A & 4.20 & 301 & 305 & 3.52 & 4.10 & 11.0 & 9.9 & \\
\hline 1164 & B3 V & 21500 & $\mathrm{E}$ & 4.00 & 190 & 217 & 1.86 & 3.87 & 8.2 & 24.5 & \\
\hline 1165 & B $1.5 \mathrm{~V}$ & 26100 & A & 4.25 & 184 & 199 & 7.02 & 4.06 & 10.3 & 13.4 & \\
\hline 1166 & B1-3e & 24950 & IM & 4.20 & 328 & 302 & 7.70 & 4.01 & 9.8 & 14.6 & \\
\hline 1167 & B1.5 V & 26100 & A & 4.05 & 117 & 119 & 2.75 & 4.05 & 10.0 & 13.9 & \\
\hline 1168 & B9 II & - & - & - & - & - & 6.20 & - & - & - & \\
\hline 1169 & B9 II & - & - & - & - & - & 9.83 & - & - & - & \\
\hline 1170 & B0.5 V & 29500 & $\mathrm{He}+$ & 4.35 & 147 & 151 & 3.76 & 4.17 & 12.0 & 7.2 & \\
\hline 1171 & A2 III-II & - & - & - & - & - & 7.21 & - & - & - & \\
\hline 1172 & B3 V & 21500 & E & 4.15 & 137 & 142 & 7.62 & 3.86 & 8.0 & 25.4 & SB1 \\
\hline 1173 & B1 V & 27300 & A & 4.20 & 75 & 72 & 4.20 & 4.08 & 10.6 & 11.7 & \\
\hline 1174 & B1-3e & 24950 & IM & 3.40 & 529 & 531 & 6.47 & 4.00 & 10.2 & 14.2 & \\
\hline 1175 & B2 V & 24950 & A & 4.40 & 103 & 96 & 8.37 & 3.99 & 9.4 & 16.6 & \\
\hline 1176 & B $1.5 \mathrm{~V}$ & 26100 & A & 4.35 & $\leq 40$ & $\leq 40$ & 7.29 & 4.03 & 9.8 & 14.6 & \\
\hline 1177 & B1-3e & 24950 & IM & 4.05 & 44 & 58 & 6.93 & 3.99 & 9.4 & 16.8 & \\
\hline 1178 & B1.5 V & 26100 & A & 4.10 & 88 & 87 & 7.50 & 4.03 & 9.8 & 14.1 & \\
\hline 1179 & B $1.5 \mathrm{~V}$ & 26100 & A & 4.20 & 133 & 134 & 4.63 & 4.03 & 10.0 & 13.8 & \\
\hline 1180 & B0.5 V & 30000 & $\mathrm{He}+$ & 4.25 & 166 & 160 & 7.90 & 4.17 & 12.2 & 5.9 & \\
\hline 1181 & B1 V & 27300 & A & 4.10 & 167 & 161 & 2.08 & 4.07 & 10.6 & 11.1 & SB1 \\
\hline 1182 & B0.5 V & 29650 & A & 4.40 & 195 & 219 & 6.40 & 4.16 & 12.2 & 6.2 & SB1 \\
\hline 1183 & B2.5 V & 23900 & $\mathrm{E}$ & 4.35 & 51 & $\leq 40$ & 9.04 & 3.93 & 8.8 & 19.9 & \\
\hline 1184 & B1-3 V & 24950 & I & 4.25 & 183 & 199 & 5.34 & 3.98 & 9.6 & 15.5 & \\
\hline 1185 & B0 V & 30500 & $\mathrm{He}+$ & 4.35 & 170 & 170 & 0.86 & 4.19 & 12.6 & 4.9 & \\
\hline 1186 & B2 V & 24950 & A & 4.05 & 41 & 41 & 4.09 & 3.98 & 9.4 & 16.3 & \\
\hline 1187 & B5e & - & - & - & 212 & 234 & 2.92 & - & - & - & \\
\hline 1188 & B1-2 V & 26100 & I & 4.15 & 310 & 294 & 3.15 & 4.01 & 10.2 & 12.1 & SB1 \\
\hline 1189 & B1 V & 27300 & A & 4.15 & 227 & 215 & 2.11 & 4.06 & 10.6 & 10.6 & \\
\hline
\end{tabular}


Table A.2. continued.

\begin{tabular}{|c|c|c|c|c|c|c|c|c|c|c|c|}
\hline \multirow[t]{2}{*}{ Star } & \multirow[t]{2}{*}{ Spectral Type } & \multirow{2}{*}{$\begin{array}{l}T_{\text {eff }} \\
(\mathrm{K}) \\
\end{array}$} & \multirow[t]{2}{*}{ Method } & \multirow{2}{*}{$\begin{array}{l}\log g \\
(\mathrm{dex}) \\
\end{array}$} & \multicolumn{2}{|c|}{$v_{\mathrm{e}} \sin i$} & \multirow{2}{*}{$\begin{array}{r}r \\
\left({ }^{\prime}\right) \\
\end{array}$} & \multirow{2}{*}{$\begin{array}{r}\log \mathrm{L} \\
\left(L_{\odot}\right)\end{array}$} & \multirow{2}{*}{$\begin{array}{l}\text { Mass } \\
\left(M_{\odot}\right) \\
\end{array}$} & \multirow{2}{*}{$\begin{array}{r}\text { Age } \\
(\mathrm{Myr})\end{array}$} & \multirow[t]{2}{*}{ Comment } \\
\hline & & & & & $\begin{array}{l}\mathrm{PF} \\
(\mathrm{kn}\end{array}$ & $\begin{array}{l}\text { FT } \\
-1)\end{array}$ & & & & & \\
\hline 1190 & B1-3e & 24950 & IM & 4.00 & 278 & 284 & 5.38 & 3.96 & 9.6 & 14.7 & \\
\hline 1191 & B $1.5 \mathrm{~V}$ & 26100 & A & 4.25 & 106 & 93 & 2.96 & 4.01 & 10.2 & 12.1 & SB1 \\
\hline 1192 & B2 V & 24950 & A & 4.20 & 128 & 123 & 8.34 & 3.97 & 9.2 & 16.5 & SB1 \\
\hline 1193 & B1-2e & 26100 & IM & 3.70 & 326 & 304 & 7.65 & 4.01 & 10.2 & 12.0 & \\
\hline 1194 & B3e & 21500 & EM & 3.80 & 204 & 196 & 1.91 & 3.82 & 7.8 & 25.3 & \\
\hline 1195 & B $1.5 \mathrm{~V}$ & 26100 & A & 4.40 & 104 & 114 & 3.62 & 4.01 & 9.8 & 13.9 & \\
\hline 1196 & B1 V & 29500 & $\mathrm{He}+$ & 4.40 & 141 & 136 & 7.02 & 4.13 & 11.8 & 6.6 & SB1 \\
\hline 1197 & B $1.5 \mathrm{~V}$ & 26100 & A & 4.05 & 147 & 140 & 3.42 & 4.01 & 9.8 & 13.7 & \\
\hline 1198 & B $1.5 \mathrm{~V}$ & 26000 & $\mathrm{He}+$ & 3.80 & 47 & 46 & 4.46 & 4.00 & 9.8 & 14.2 & \\
\hline 1199 & B0 V & 34750 & $\mathrm{He}+$ & 4.40 & $\leq 40$ & $\leq 40$ & 0.94 & 4.32 & 15.6 & 0.0 & \\
\hline 1200 & B1 V & 27300 & A & 4.10 & $\leq 40$ & $\leq 40$ & 6.74 & 4.04 & 10.2 & 11.9 & \\
\hline 1201 & B $2.5 \mathrm{~V}$ & 23900 & $\mathrm{E}$ & 4.35 & 92 & 105 & 4.00 & 3.91 & 8.8 & 19.3 & \\
\hline 1202 & A0 II & - & - & - & - & - & 7.65 & - & - & - & \\
\hline 1203 & B2-3e (shell) & 23900 & EM & 3.95 & 327 & 298 & 8.78 & 3.90 & 9.0 & 17.0 & \\
\hline 1204 & A3 III & - & - & - & - & - & 2.77 & - & - & - & \\
\hline 1205 & B2.5 V & 23900 & $\mathrm{E}$ & 4.10 & 107 & 108 & 7.94 & 3.89 & 8.6 & 19.3 & \\
\hline 1206 & B2.5 V & 23900 & $\mathrm{E}$ & 4.20 & 42 & 46 & 3.88 & 3.89 & 8.6 & 19.3 & \\
\hline 1207 & B3 V & 21500 & $\mathrm{E}$ & 4.25 & $\leq 40$ & $\leq 40$ & 5.60 & 3.79 & 7.6 & 27.2 & \\
\hline 1208 & B2.5 V & 23900 & $\mathrm{E}$ & 4.05 & 114 & 128 & 7.04 & 3.89 & 8.6 & 19.1 & \\
\hline 1209 & B3 V & 21500 & $\mathrm{E}$ & 3.85 & 132 & 145 & 6.04 & 3.78 & 7.6 & 26.4 & SB1 \\
\hline 1210 & B3e (shell) & 21500 & EM & 3.40 & 234 & 238 & 6.83 & 3.78 & 7.8 & 25.2 & \\
\hline 1211 & B1.5 V & 26100 & A & 4.20 & 241 & 243 & 4.55 & 3.97 & 9.8 & 12.4 & \\
\hline 1212 & B3e & 21500 & EM & 4.15 & 300 & 307 & 3.30 & 3.78 & 8.0 & 24.0 & \\
\hline 1213 & B1-3e & 24950 & $\mathrm{IM}$ & 4.00 & 309 & 304 & 6.87 & 3.92 & 9.4 & 14.3 & \\
\hline 1214 & B1-3e & 24950 & IM & 3.90 & 351 & 336 & 4.96 & 3.92 & 9.4 & 14.3 & \\
\hline 1215 & B3 III & 18450 & A & 3.45 & 96 & 87 & 8.25 & 3.62 & 6.4 & 41.2 & \\
\hline 1216 & B $1.5 \mathrm{~V}$ & 26100 & A & 4.15 & 251 & 272 & 2.13 & 3.95 & 9.8 & 11.9 & \\
\hline 1217 & B2.5 V & 23900 & $\mathrm{E}$ & 4.15 & $\leq 40$ & $\leq 40$ & 5.90 & 3.86 & 8.4 & 20.0 & \\
\hline 1218 & B2.5 V & 23900 & $\mathrm{E}$ & 3.85 & 266 & 266 & 6.35 & 3.86 & 8.8 & 17.4 & \\
\hline 1219 & B1.5 V & 26100 & A & 4.30 & 83 & 99 & 4.57 & 3.95 & 9.6 & 13.6 & \\
\hline 1220 & $\mathrm{~B} 1.5 \mathrm{~V}$ & 26100 & A & 4.20 & 160 & 155 & 2.27 & 3.95 & 9.6 & 13.2 & \\
\hline 1221 & B $0.5 \mathrm{~V}$ & 29200 & $\mathrm{Si}$ & 4.25 & $\leq 40$ & $\leq 40$ & 2.81 & 4.06 & 11.2 & 6.8 & \\
\hline 1222 & B1 V & 27300 & A & 4.30 & 259 & 265 & 3.97 & 3.98 & 10.4 & 8.9 & \\
\hline 1223 & B1 V & 27300 & A & 4.30 & 47 & 55 & 6.29 & 3.98 & 10.2 & 10.8 & \\
\hline 1224 & B1-2e & 26100 & IM & 4.00 & 247 & 238 & 7.43 & 3.94 & 9.8 & 12.2 & \\
\hline 1225 & B3 V & 21500 & $\mathrm{E}$ & 4.20 & 96 & 79 & 5.84 & 3.75 & 7.4 & 27.4 & \\
\hline 1226 & B $1.5 \mathrm{~V}$ & 26100 & A & 4.15 & 94 & 99 & 6.62 & 3.94 & 9.6 & 13.6 & \\
\hline 1227 & B3 V & 21500 & $\mathrm{E}$ & 4.05 & 54 & 68 & 8.54 & 3.75 & 7.4 & 27.5 & \\
\hline 1228 & B9 II & - & - & - & - & - & 9.15 & - & - & - & \\
\hline 1229 & B $1.5 \mathrm{~V}$ & 26100 & A & 4.30 & 194 & 212 & 4.78 & 3.93 & 9.6 & 12.3 & \\
\hline 1230 & B0.5 V & 29650 & A & 4.25 & 199 & 198 & 3.74 & 4.06 & 11.8 & 3.9 & \\
\hline 1231 & B8 II & 12500 & $\mathrm{He}$ & 3.15 & 66 & 79 & 2.26 & 3.19 & - & - & \\
\hline 1232 & B1 V & 27300 & A & 4.45 & 149 & 135 & 0.55 & 3.97 & 10.2 & 10.1 & \\
\hline 1233 & B0.2 V & 30650 & $\mathrm{Si}$ & 4.40 & $\leq 40$ & $\leq 40$ & 3.22 & 4.10 & 12.0 & 3.0 & \\
\hline 1234 & B2.5 V & 23900 & $\mathrm{E}$ & 4.00 & $\leq 40$ & $\leq 40$ & 2.92 & 3.84 & 8.4 & 20.0 & \\
\hline 1235 & B3 V & 21500 & $\mathrm{E}$ & 4.15 & 236 & 213 & 5.46 & 3.74 & 7.6 & 25.9 & \\
\hline 1236 & B1 V & 27000 & $\mathrm{He}+$ & 4.30 & 69 & 67 & 1.17 & 3.96 & 10.2 & 9.0 & \\
\hline 1237 & $\mathrm{~B} 3 \mathrm{e}$ & 21500 & EM & 4.00 & 200 & 220 & 8.63 & 3.74 & 7.6 & 25.9 & \\
\hline 1238 & B2.5 V & 23900 & $\mathrm{E}$ & 3.90 & 135 & 141 & 5.62 & 3.84 & 8.4 & 19.0 & \\
\hline 1239 & B2 V & 24950 & A & 4.35 & 44 & 41 & 7.37 & 3.88 & 8.8 & 16.8 & \\
\hline 1240 & B3 V & 21500 & E & 3.70 & 272 & 262 & 6.49 & 3.73 & 7.6 & 25.2 & \\
\hline 1241 & B1 V & 27300 & A & 4.35 & 94 & 99 & 2.31 & 3.96 & 10.2 & 10.1 & SB1 \\
\hline 1242 & B2 V & 24950 & A & 4.50 & 280 & 298 & 6.11 & 3.87 & 9.2 & 13.9 & \\
\hline 1243 & B3 V & 21500 & $\mathrm{E}$ & 4.15 & 65 & 70 & 4.02 & 3.73 & 7.4 & 27.8 & \\
\hline 1244 & B $2.5 \mathrm{~V}$ & 23900 & E & 3.95 & 120 & 135 & 1.53 & 3.82 & 8.4 & 19.1 & \\
\hline 1245 & B $1.5 \mathrm{~V}$ & 26100 & A & 4.15 & 148 & 133 & 6.41 & 3.91 & 9.4 & 13.0 & \\
\hline 1246 & B3 V & 21500 & $\mathrm{E}$ & 4.10 & 131 & 119 & 6.33 & 3.72 & 7.4 & 27.4 & SB1 \\
\hline 1247 & B $1.5 \mathrm{~V}$ & 26100 & A & 4.20 & 188 & 187 & 0.39 & 3.90 & 9.6 & 12.3 & \\
\hline
\end{tabular}




\section{Appendix B: Comments on individual targets}

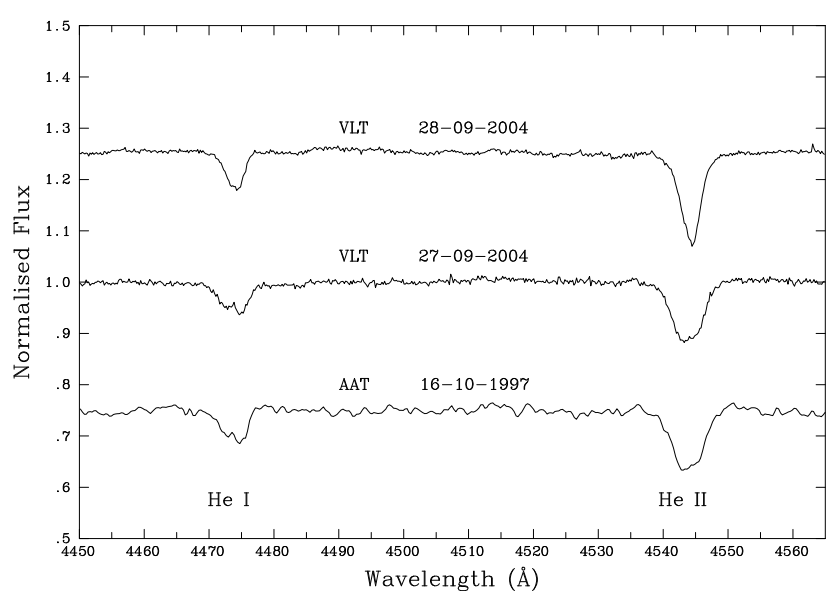

Fig. B.1. \#1001 - MPG 435. Multiple components are seen in the FLAMES spectra. Evidence for multiplicity is also seen in an unpublished AAT-UCLES spectrum (degraded and binned to the same resolution as the FLAMES data).

\#1001 - MPG 435. This is the visually brightest object in the main body of NGC 346, first observed spectroscopically by Walborn (1978, his "NGC 346 No. 1") and classified as O4 III(n)(f). From consideration of its absolute magnitude, and from the offset of the stellar radial velocities compared to the nebular emission features, Niemela et al. (1986) suggested it as a likely binary. Indeed, Niemela (2002) reported radial velocity variations in the system (classifying the spectra as $\mathrm{O} 4 \mathrm{f}+\mathrm{O}:$ ), with a refined estimate of the period of $24.2 \mathrm{~d}$ given by Niemela \& Gamen (2004). The FLAMES data, observed as part of the Field A configuration, also reveal a massive companion. Note that this is probably not related to the multiple components reported by Heydari-Malayeri \& Hutsemekers (1991), which are at distances of greater than 1'.5 (thus outside the Medusa fibre diameter).

The $\lambda \lambda 4450-4565$ region of \#1001 is shown for our two LR02 spectra in Fig. B.1. Double components are clearly seen in the data from 2004 September 27, whereas the observation from the following night merely shows line asymmetry - such a rapid change for a relatively long-period system is consistent with the eccentric orbit ( $e=0.42 \pm 0.04$ ) from Niemela \& Gamen (2004). For completeness we also include a previously unpublished spectrum of the system from the University College London Echelle Spectrograph (UCLES) on the Anglo Australian Telescope (AAT), contemporary to those presented by Walborn et al. (2000) - similar evidence of multiplicity is also seen.

$\mathrm{N}$ IV $\lambda 4058$ emission (requiring a spectral type of $\mathrm{O} 4$ or earlier) is seen in the LR02 spectra at a velocity consistent with the blueward component in Fig. B.1. The presence of He I $\lambda 4471$ absorption, combined with weak He II $\lambda 4686$ emission (albeit complicated by absorption from the secondary) suggests a classification for the primary of $\mathrm{O} 4$ If. The $\mathrm{He}$ I $\lambda 4471$ absorption for the secondary is also weaker than that for He II $\lambda 4542$ (Fig. B.1), suggesting a classification of 05-6.

\#1010 - MPG 342. Niemela et al. (1986) also suggested this star as a likely binary, with further monitoring by Massey et al. (2012) revealing a third component. The FLAMES spectra, obtained in the Field B configuration, are best described as O5$6 \mathrm{~V}((\mathrm{f}))$, but the multiplicity complicates precise classification and prompted us to re-inspect another unpublished AAT-UCLES spectrum (again contemporary to those from Walborn et al.

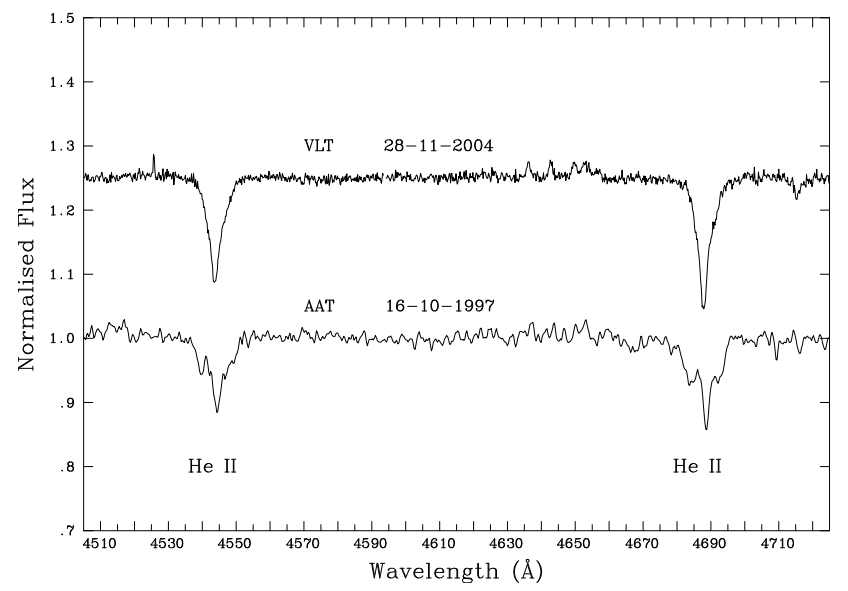

Fig. B.2. \#1010 - MPG 342. LR03 FLAMES spectrum in which \#1010 appears single, compared with a previously unpublished AAT-UCLES spectrum (degraded/binned to the FLAMES resolution), in which three components appear to be present.

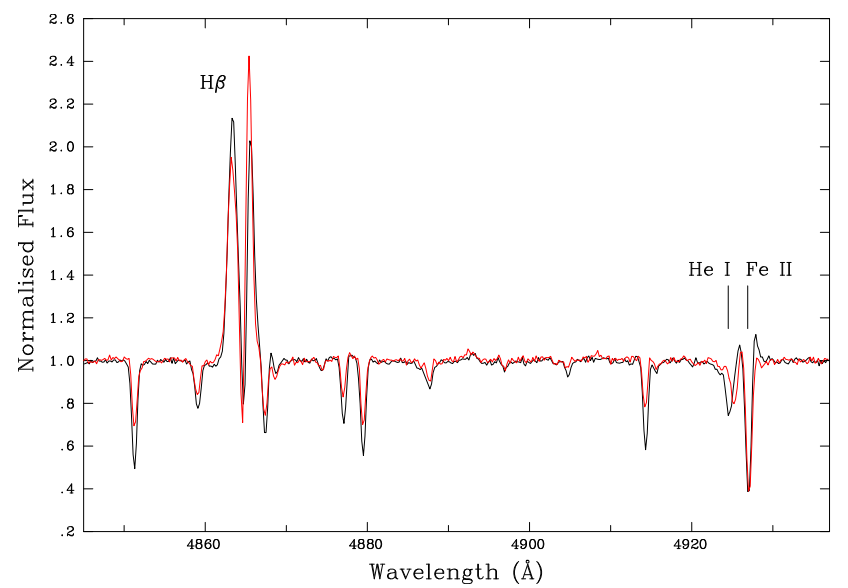

Fig. B.3. Combined LR03 observations for \#1024 from the two observational epochs. The spectrum from the first epoch (black line) has been redshifted by $15 \mathrm{~km} \mathrm{~s}^{-1}$ such that the metallic spectra approximately agree - note the offset in the He I $\lambda 4922$ absorption, indicative of a hotter companion, and the shell-like emission of $\mathrm{Fe}$ II $\lambda 4923$.

2000) which also shows evidence of three components (see Fig. B.2).

\#1012 - MPG 368. As one of the brightest stars in the cluster, \#1012 has been observed by a number of authors. Classifications of O5-6: V, O5.5 V((f+)), O4-5 V((f)) and O6 V were given by Niemela et al. (1986), Massey et al. (1989), Walborn et al. (2000), and Massey et al. (2009), respectively. Massey et al. reported a velocity difference $\left(50 \mathrm{~km} \mathrm{~s}^{-1}\right)$ between the He I and II lines in their spectrum of the star and suggested it is a binary, with more recent high-resolution spectroscopy from Bouret et al. (2013) finding evidence of two components in the He I lines. The FLAMES spectra also reveal a velocity shift (of $\sim 50 \mathrm{~km} \mathrm{~s}^{-1}$ ) within the observations, providing further support of its binary nature (hence the past uncertainty in its classifications).

\#1024 - MPG 665, MA93\#1138, KWB346\#93. This source was noted as an emission-line object by Meyssonnier \& Azzopardi (1993, their \#1138) then by Keller etal. (1999, \#93) in their search for Be-type stars; on the basis of imaging polarimetry, Wisniewski et al. (2007) argued that it is most likely a classical Be star. However, \#1024 was identified by Udalski et al. (1998, 
SMC_SC8,\#160677) as an eclipsing binary system from the second Optical Gravitational Lensing Experiment (OGLE) survey, with a period of $86.4 \mathrm{~d}$, updated to $86.2 \mathrm{~d}$ by Wyrzykowski et al. (2004) and then $86.25 \mathrm{~d}$ by Pawlak et al. (2013).

Strong twin-peaked emission is seen in $\mathrm{H} \beta$, with weak $\mathrm{He}_{\mathrm{I}}$ absorption combined with a rich metal-line absorption spectrum super-imposed (consistent with a late A spectral type). This initially suggested a Be-type object with a cooler disk. The timesampling of our data is unfortunately relatively limited (\#1024 was observed in Field A), but the LR03 observations were taken three nights apart and variations are seen in the $\mathrm{H} \beta$ region (see Fig. B.3). The first observation (2004 Oct 01) has been redshifted by $15 \mathrm{~km} \mathrm{~s}^{-1}$ such that the metal absorption lines roughly match those in the second observation; note the residual offset in He $\mathrm{I} \lambda 4922\left(\sim 40 \mathrm{~km} \mathrm{~s}^{-1}\right)$ and shell-like emission in Fe II $\lambda 4923$. Similar behaviour is also seen in He I $\lambda 4713$ and $\lambda 5016$ but the velocity of the $\mathrm{H} \beta$ emission appears consistent with the cooler spectrum. The blue:red ratio of the $\mathrm{H} \beta$ emission changes, perhaps related to relative shifts in the line profile of the hotter companion rather than arising from the longer-term changes often seen and thought to arise from disk structure (Hanuschik et al. 1995). This system merits further study to ascertain its evolutionary status, but could be related to the peculiar A-type supergiants reported by Mennickent \& Smith (2010).

\#1030 - MPG 655. The spectrum of \#1030 (MPG 655) has very narrow lines, similar to those seen in NGC 346-028 from the FSMS (aka MPG 113), classified as OC6 Vz by Walborn et al. (2000). The He I lines suggest a slightly earlier type for \#1030, but there is also evidence of nebular infilling, so we classify its spectrum as OC5-6 Vz. From lower-resolution spectroscopy, Heydari-Malayeri \& Selier (2010) classified this star as $\mathrm{O} 5 \mathrm{~V}+\mathrm{OB}$, with the suggestion of a secondary component arising from the strength of the $\mathrm{He}$ I lines given an $\mathrm{O} 5 \mathrm{~V}$ classfication (Dr. N. R. Walborn, priv. comm.); from our (albeit limited) time coverage, we see no evidence for radial velocity shifts. If the spectrum is convolved by a rotational broadening profile of, for example, $200 \mathrm{~km} \mathrm{~s}^{-1}$, the He II $\lambda 4686$ line still remains stronger than the other helium lines, suggesting its classification as "Vz" spectrum is not due to either resolution or $v_{\mathrm{e}} \sin i$ effects. 